\title{
Notes on the Langevin Model for Turbulent Diffusion of "Marked" Particles
}

Howard C. Rodean

January 26, 1994

1. This is an informal report intended primarily for internal or limited external distribution. The opinions and conclusions stated are those of the author and may or may not be those of the Laboratory.

Work performed under the auspices of the U.S. Department of Energy by the Lawrence Livermore National Laboratory under Contract W-7405-Eng-48. 


\section{DISCLAIMER}

This document was prepared as an acccount of work sponsored by an agency of the United States Government. Neither the United States Government nor the University of California nor any of their employees, makes any warranty, express or implied, or assumes any legal liability or responsibility for the accuracy, completeness, or usefulness of any information, apparatus, product, or process disclosed, or represents that its use would not infringe privately own rights. Reference herein to any specifie commercial products, process, or service by trade name, trademark, manufacturer, or otherwise, does not necessarily constitute or imply its endorsement, recommendation, or favoring by the United States Government or the University of California. The views and opinions of authors expressed herein do not necessarily state or reflect those of the United States Government or the University of California, and shall not be used for advertising or product endorsement purposes.

This report has been reproduced directly from the best available copy.

Available to DOE and DOE contractors from the Office of Scientific and Technical Information P.O. Box 62, Oak Ridge, IN 37831

Prices available from (615) 576-8401, FTS 626-8401

Available to the public from the National Technical Information Service

U.S. Department of Commerce 5285 Port Royal Rd., Springfield, VA 22161 
Notes on the Langevin Model for Turbulent Diffusion of "Marked" Particles

Howard C. Rodean 


\section{PREFACE}

These "Notes on the Langevin Model ..." are, in a sense, a scientific diary. This story began in December 1988 when I proposed that my principal pre-retirement assignment be the implementation of the Langevin model for turbulent scalar diffusion. I was able to spend much of my time during 1989 on the study of the Langevin model. I was spending essentially all my time on this subject by the time I partially retired in March 1990 . I was invited to continue as a Laboratory Associate in order to bring this work to some reasonably well-defined state of "completion." My responsibilities increased substantially in October 1991 when I became a principal investigator for joint US-USSR (now Russia) scientific collaboration on atmospheric dispersion modeling. My colleague is Vladimir P. Gavrilov of the Institute of Experimental Meteorology in Obninsk, Russia. This work is a valuable complement to my origina! assignment.

These Notes consist of three parts and an appendix. Part II is the oldest with dates from November 1989 through October 1990 on the final version of the handwritten manuscript. It is a record of my learning process as I studied the Langevin equation and related material on the mathematical theory of stochastic processes. This study confirmed my initial opinion that (1) the Langevin equation, the archetype of stochastic differential equations, is very different from classical differential equations and (2) care must be taken in order to apply it correctly. The sequence of pages in Part II reflects the histories of both the Langevin equation and my course of study. Part I is based on a manuscript dated January 1992 which was written as my proposed version of the first part of a joint work with Vladimir Gavrilov. It may be regarded as an executive summary of Part II written from a later perspective. Part III is based on my work with colleagues to implement the Langevin model for scalar diffusion in turbulent flow. Major topics include (1) the forms of the Langevin model for one, two, and three dimensions; and (2) parameterizations of turbulence statistics for use as inputs to the model for the full range of conditions in the atmospheric boundary layer. These Notes conclude with the Appendix in which two of the possible nonunique solutions of the Fokker-Planck equation are derived in detail for velocity fluctuations in three-dimensional, nonstationary, inhomogeneous turbulence. The results are two mathematically valid forms of the Langevin model for scalar diffusion in turbulent flow.

These Notes have served one purpose as I wrote them, and they are intended to serve another in the future. Long ago, I began the practice of writing as I studied a new subject in order to make sure that I was gaining a real understanding. An early example is my only book, Nuclear-Explosion Seismology (USAEC, Washington DC, 1971; Russian translation "Mir," Moscow, 1974), which had its beginning in the late 1960s when I knew nothing about seismology and a little about the physics of underground nuclear explosions. These Notes served the same purpose in helping me learn about the Langevin model. In the future, I hope, they will serve as a means of "technology transfer" to my younger colleagues because Laboratory Associates, in time, completely retire. 


\section{Contents}

PART I: Comparison of Three Models for Scalar Diffusion in Turbulent Flow . . . . . . 1 Abstract ............................ 1

Introduction . . . . . . . . . . . . . . . . . . . . . . . . . . 1

Model Descriptions and Interrelations . . . . . . . . . . . . . . . . . . . . . 3

The Langevin Model . . . . . . . . . . . . . . . . . . . . . . . . 3

The Random Displacement Model . . . . . . . . . . . . . . . . . . . . . . . 8

The Eddy Diffusivity Model . . . . . . . . . . . . . . . . . . . . . . . . 11

Model Inputs . . . . . . . . . . . . . . . . . . . . . . . . . . . . . . . 12

Applicability of Each Model . . . . . . . . . . . . . . . . . . . . 12

References . . . . . . . . . . . . . . . . . . . . . . . . 13

PART II: Studies of the Langevin Model, Its Applications, and the Theory of

Stochastic Processes . . . . . . . . . . . . . . . . . . . . . 16

Abstract . . . . . . . . . . . . . . . . . . . . . . . . . 16

Introduction . . . . . . . . . . . . . . . . . . . . . . . . . . 16

Brownian Motion . . . . . . . . . . . . . . . . . . . . . . . . . 16

Turbulent Diffusion . . . . . . . . . . . . . . . . . . . . . . . . . . . 17

The Langevin Equation for Turbulent Diffusion . . . . . . . . . . . . . . . . . 18

Physical Reinterpretation . . . . . . . . . . . . . . . . . . . . . . . 18

Diffusion in Stationary Homogeneous Turbulence . . . . . . . . . . . . . . . 19

From "White Noise" to the "Wiener Process" . . . . . . . . . . . . . . . . 21

Drift Corrections for Inhomogeneous Turbulence . . . . . . . . . . . . . . . 23

Derivation of Drift Corrections . . . . . . . . . . . . . . . . . . . . . . 25

Gaussian and Non-Gaussian Distributions . . . . . . . . . . . . . . . . . . 33

Realizable Probability Distributions . . . . . . . . . . . . . . . . . . . . . 34

Definitions of $\sigma_{w}$ and $\tau_{L}$ for Inhomogeneous Turbulence . . . . . . . . . . . . 36

Interim Review and References . . . . . . . . . . . . . . . . . . . . . . . . 38

Review . . . . . . . . . . . . . . . . . . . 38

Postscript . . . . . . . . . . . . . . . . . . . . . 41

References . . . . . . . . . . . . . . . . . . . . . . . . 42

Two "Relatives" of the Langevin Equation . . . . . . . . . . . . . . . . . . . 44

The Markov or Diffusion Equation Limit . . . . . . . . . . . . . . . . . . . 44

The Fokker-Planck Equation: Introduction . . . . . . . . . . . . . . . . . . 48

The Fokker-Planck Equation: Derivation . . . . . . . . . . . . . . . . . . . 50

The Fokker-Planck Equation: Relation to the Langevin Equation . . . . . . . . 55

References . . . . . . . . . . . . . . . . . . . . . 55

Present Applications of the Langevin Model . . . . . . . . . . . . . . . . . . 57

Introduction . . . . . . . . . . . . . . . . . . . . . . . . . 57

The Surface Boundary Layer . . . . . . . . . . . . . . . . . . . . . . . 57

The Convective Boundary Layer . . . . . . . . . . . . . . . . . . . . . . 62

Parameterization of the Time Scale . . . . . . . . . . . . . . . . . . . . . 67

The Lagrangian Structure Function and its "Universal" Constant . . . . . . . . 69

Other Turbulence Parameterizations . . . . . . . . . . . . . . . . . . . . . 70

Boundary Conditions . . . . . . . . . . . . . . . . . . . . . . . . . . 75

Experimental Data Sets and Test Problems . . . . . . . . . . . . . . . . . 76

Summary .. . . . . . . . . . . . . . . . . . . . . . . 78

References .. . . . . . . . . . . . . . . . . 80 
PART III: Toward Implementation of Stochastic Models for Diffusion

Abstract . . . . . . . . . . . . . . . . . . . . . . . . . . . . 84

Introduction . . . . . . . . . . . . . . . . . . . . . . . . . . . . . . . . 84

The Langevin and Random Displacement Models for Diffusion . . . . . . . . . . . 84

The Relation between the Langevin and Random Displacement Models . . . . . 85

Ranges of Time $t$ and Time Increments $\Delta t$ for the Two Models . . . . . . . . . 85

Langevin Models for Diffusion in Gaussian Turbulence . . . . . . . . . . . . . 86

"Simplest" Model for Diffusion in Three Dimensions . . . . . . . . . . . . . . . 87

Reduction for Diffusion in Two Dimensions . . . . . . . . . . . . . . . . . . 89

Reduction for Diffusion in One Dimension . . . . . . . . . . . . . . . . . . . 89

Random Displacement Models for Diffusion in Gaussian Turbulence . . . . . . . 90

Langevin Models for Diffusion in Non-Gaussian Turbulence . . . . . . . . . . . 90

Model for One-Dimensional Diffusion in the Convective Boundary Layer . . . . . 90

Appendix: Reduction to Gaussian Turbulence . . . . . . . . . . . . . . . . 93

Extension to Three-Dimensional Diffusion . . . . . . . . . . . . . . . . . . 95

Parameterization of Turbulence Statistics for Use as Model Inputs . . . . . . . . 95

Introduction . . . . . . . . . . . . . . . . . . . . . . . . . . . . . . . 95

Basic Scaling Parameters . . . . . . . . . . . . . . . . . . . . . . . 96

Vertical Velocity Variance $\sigma_{w}^{2}$. . . . . . . . . . . . . . . . . . . . . . 97

Rate of Dissipation of Turbulence Kinetic Energy $\varepsilon$. . . . . . . . . . . . . . 99

Skewness of the Vertical Velocity $\left\langle w^{3}\right\rangle / \sigma_{w}^{3}$. . . . . . . . . . . . . . . . . . 101

Horizontal Velocity Variances $\sigma_{u}^{2}$ and $\sigma_{v}^{2}$. . . . . . . . . . . . . . . . . . . 101

Shear Stress $\tau_{u w}$. . . . . . . . . . . . . . . . . . . . . . . . . . . . . 102

Finite-Difference Forms of the Models . . . . . . . . . . . . . . . . . . . . . 103

The Ito Calculus for Stochastic Differential Equations . . . . . . . . . . . . . 103

Example: Finite-Difference Equations for 1D Diffusion . . . . . . . . . . . . . 104

Summary . . . . . . . . . . . . . . . . . . . . . . . . . . . . 105

Acknowledgements . . . . . . . . . . . . . . . . . . . . . . . 105

References . . . . . . . . . . . . . . . . . . . . . . . 107

APPENDIX: From Eq. (4) toward Eq. (32) in Thomson (1987) . . . . . . . . . . . 110

Introduction . . . . . . . . . . . . . . . . . . . . . . . . . . . . 110

Multivariate Gaussian Distribution for Turbulence . . . . . . . . . . . . . . 110

Brief Summary of Thomson's Developemnt of the "Simplest" Solution . . . . . . 112

Solutions for Turbulent Diffusions in Three Dimensions . . . . . . . . . . . . . 114

Partical Derivatives of the Multivariate Gaussian Distribution . . . . . . . . . 114

The Solution for the "Fading Memory" Term . . . . . . . . . . . . . . . . . 115

Solutions for Components of the "Drift" Term . . . . . . . . . . . . . . . . 115

The Borgas and Thomson Solutions . . . . . . . . . . . . . . . . . . . . . 120

Reduction to One-Dimensional Turbulence . . . . . . . . . . . . . . . . . . . 120

References . . . . . . . . . . . . . . . . . . . . . . 122 


\title{
Part I: Comparison of Three Models for Scalar Diffusion in Turbulent Flow
}

\begin{abstract}
Three models for scalar diffusion in turbulent flow (eddy diffusivity, random displacement, and random velocity increment based on the Langevin equation) are briefly described. These models are then examined in more detail in the reverse order. The Fokker-Planck equation is introduced as the Eulerian equivalent of the Lagrangian Langevin equation, and the derivation of each equation is outlined. The procedure for obtaining the deterministic and stochastic components of the Langevin equation from Kolmogorov's 1941 inertial range theory and the Fokker-Planck equation is described. It is noted that a unique form of the Langevin equation can be determined for diffusion in one dimension but not in two or three. The Langevin equation for vertical diffusion in the non-Gaussian convective boundary layer is presented and successively simplified for Gaussian inhomogeneous turbulence and Gaussian homogeneous turbulence in turn. The Langevin equation for Gaussian inhomogeneous turbulence is mathematically transformed into the random displacement model. It is shown how the Fokker-Planck equation for the random displacement model is identical in form to the partial differential equation for the eddy diffusivity model. It is noted that the Langevin model is applicable in two cases in which the other two are not valid: (1) very close in time and distance to the point of scalar release and (2) the non-Gaussian convective boundary layer. The two- and three-dimensional cases are considered in Part III.
\end{abstract}

\section{Introduction}

Many kinds of models have been developed to simulate the diffusion of scalars in the turbulent atmospheric boundary layer. Some are simple empirical parameterizations and others are empirical modifications of theoretical models. In this work, we study and compare three models of the turbulent diffusion process: the Eulerian eddy diffusion, the equivalent Langrangian random displacement, and the Langrangian random velocity increment based on the Langevin equation. We begin by describing these models and demonstrating how they are related to each other. We review the available data bases and parameterizations of turbulence statistics that are used as inputs to these models. We describe a number of test problems and present results from these models for a wide range of atmospheric stability conditions. We compare the performance of these models and identify the model that is, at present, most appropriate for use in each of the major atmospheric stability regimes from the convective boundary layer to the stable surface layer. The relative ranking of these models for a given condition could change as a result of changes in the quality and quantity of input data.

We make two assumptions in this study. First, we assume constant-density (but not incompressible) flow which results in simpler equations. Second, we assume stationary, horizontallyhomogeneous turbulence and consider turbulent diffusion in only the vertical dimension. We consider only the one-dimensional case of vertical diffusion because (1) it has been the most thoroughly 
investigated, and (2) there are some problems that have not been solved for two and three dimensions.

The eddy diffusivity model is based on the Reynolds-averaged equation for the transport of a scalar quantity $\theta$ in a constant-density fluid (Monin and Yaglom, 1971, Secs. 5.1 and 10.3):

$$
\frac{\partial \Theta}{\partial t}+U_{i} \frac{\partial \Theta}{\partial x_{i}}=\frac{\partial}{\partial x_{i}}\left(k \frac{\partial \Theta}{\partial x_{i}}-\left\langle u_{i} \theta\right\rangle\right)
$$

where $\Theta$ and $U_{i}$ are, respectively, the mean values of the scalar and Eulerian velocity, $\theta$ and $u_{i}$ are the respective fluctuations from the mean, $k$ is the molecular coefficient of diffusion, and $\left\langle u_{i} \theta\right\rangle$ is the turbulent flux of the scalar in the $i$ direction. In this model, it is assumed that

$$
\left\langle u_{i} \theta\right\rangle=-K \partial \Theta / \partial x_{i}
$$

where $K$ is the coefficient of eddy diffusivity. The scalar may be temperature or a substance like water vapor or a pollutant. The molecular diffusivity is generally negligible compared to this turbulent diffusion, so we combine Eqs. (1) and (2) to get the diffusion equation,

$$
\frac{\partial \Theta}{\partial t}+U_{i} \frac{\partial \Theta}{\partial x_{i}}=\frac{\partial}{\partial x_{i}}\left(K \frac{\partial \Theta}{\partial x_{i}}\right)
$$

This equation is the basis for the eddy diffusivity model.

The random displacement model is similar to the random walk model that Einstein used in 1905 in his work on Brownian motion (Gardiner, 1983, pp. 2-6). Chandrasekhar demonstrated in $\mathrm{Ch}$. I of his 1943 paper that, in the limit of a large number of random steps, this model approaches the diffusion equation. We will follow the work of Boughton et al. (1987) who proposed this equation for turbulent diffusion (but only for the vertical dimension):

$$
d x_{i}=a_{i}(\boldsymbol{x}, t) d t+b_{i j}(\boldsymbol{x}, t) d W_{j}(t)
$$

In this three-dimensional version of their model, $\boldsymbol{x}_{\boldsymbol{i}}$ is the location of a "marked particle" on the $i$ coordinate, $a_{i}(\boldsymbol{x}, t) d t$ is a deterministic term and $b_{i j}(\boldsymbol{x}, t) d W(t)$ is a stochastic term. The coefficients $a_{i}$ and $b_{i j}^{2}$ are, respectively, the coefficients of drift and diffusion (Arnold, 1974. pp. xiii). The stochastic term models a Markov process in which "the past and future are statistically independent when the present is known" (Arnold, p. 29). The quantity $d W_{j}(t)$ is an incremental Wiener process (Arnold, Sec. 3.1; Gardiner, Sec. 3.8.1). This process is integrable but is everywhere discontinuous. The distribution of $d W_{j}(t)$ is Gaussian with mean zero and variance $d t$; in more than one dimension $d W_{i}(t)$ and $d W_{j}(s)$ are statistically independent if $i \neq j$ with $s=t$ or if $i=j$ with $s \neq t$ :

$$
\begin{gathered}
\left\langle d W_{j}(t)\right\rangle=0 \\
\left\langle d W_{i}(t) d W_{j}(s)\right\rangle=\delta_{i j} \delta(t-s) d t d s
\end{gathered}
$$

where $\delta_{i j}$ is the Kronecker delta and $\delta(t-s)$ is the Dirac delta function.

The random velocity increment model is based on the equation that Langevin proposed in 1908 as a model for Brownian motion (Gardiner, 1983, pp. 6-8). Its modern use as a model for turbulent diffusion is a consequence of Obukhov's (1959) suggestion that the Fokker-Planck equation is the Eulerian equivalent of the Langevin equation which is Lagrangian. Obukhov's suggestion led to a 
equation in papers that appeared in the 1960s, 1970s, and early 1980s. More recently, the FokkerPlanck equation has been used as a complement to the Langevin equation in modeling turbulent diffusion; we will do likewise in this study. Thomson (1987) is a definitive paper on the Langevin model which he wrote in the form

$$
\begin{aligned}
d u_{i} & =a_{i}(\boldsymbol{x}, \boldsymbol{u}, t) d t+b_{i j}(\boldsymbol{x}, \boldsymbol{u}, t) d W_{j}(t) \\
d \boldsymbol{x} & =\boldsymbol{u} d t
\end{aligned}
$$

Here $\boldsymbol{u}$ is the Lagrangian velocity of a marked particle and all the other symbols have the same meaning as in Eqs. (4) and (5a-b).

Equations (4) and (6a-b) are stochastic differential equations that, when integrated, define the trajectory of a particle in space. These equations are used to calculate the independent trajectories of thousands of particles to simulate the turbulent diffusion of a cloud, and the volumetric concentration of the particles is used as an approximation to the mean scalar quantity $\Theta$ in Eq. (3). We note that these stochastic differential equations are to be integrated according to the rules of the Ito Calculus (Arnold, 1974, pp. 58-61; Gardiner, 1983, pp. 83-92). On the other hand, Eq. (3) is to be integrated according to the rules of the classical Riemann-Stieltjes Calculus.

\section{Model Descriptions and Interrelations}

We now examine in detail the three models described by Eqs. (3)-(6a-b) and show how they are related to each other. We will study them in the reverse order because the Langevin model can be mathematically transformed into the random displacement model which, in turn, is closely related to the eddy diffusivity model. We will also consider the Fokker-Planck equation equivalent of these models. In the remainder of this paper, we assume stationary, horizontally-homogeneous turbulent flow in which the mean lateral $\left(\left\langle u_{2}\right\rangle\right.$ or $\left.\langle v\rangle\right)$ and vertical $\left(\left\langle u_{3}\right\rangle\right.$ or $\left.\langle w\rangle\right)$ flow velocities are zero.

\section{The Langevin Model}

Much of the following is based on the definitive paper of Thomson (1987). We rewrite Eqs. $(6 \mathrm{a}-\mathrm{b})$ in terms of the vertical Lagrangian velocity $w$ and the vertical coordinate $z$ :

$$
\begin{aligned}
d w & =a(z, w) d t+b(z, w) d W(t) \\
d z & =w d t
\end{aligned}
$$

The generalized forward Kolmogorov or Fokker-Planck equation for this system is

$$
\frac{\partial P}{\partial t}+\frac{\partial}{\partial z}(w P)=-\frac{\partial}{\partial w}(a P)+\frac{1}{2} \frac{\partial^{2}}{\partial w^{2}}\left(b^{2} P\right)
$$

where $P$ is the probability density function in position and velocity space ( $z, w$ space) of all the marked particles in the ensemble of flows. We assume stationary turbulence so the first term on the left is equal to zero. We will use $\partial P / \partial t=0$ in the remainder of this work with the Langevin model-but there will be some exceptions with $\partial P / \partial t \neq 0$ involving other models. 
The derivation of the basic form of the Langevin equation is relatively simple and is based on physical reasoning: the time-dependent velocity of a particle in space is a function of a deterministic dynamic component and a fluctuating random component; these are respectively represented by the first and second terms on the right side of Eq. (7a). The drift and diffusion coefficients, $a$ and $b^{2}$ respectively, are functions of the first and second moments of Eq. (7a) in combination with the properties of the incremental Wiener prosess given by Eqs. (5a-b) (Doob, 1958, Sec. VI.3; Arnold, 1974, Sec. 2.5):

$$
\begin{gathered}
a(z, w)=\langle d w\rangle / d t, \\
b^{2}(z, w)=\left\langle(d w)^{2}\right\rangle / d t .
\end{gathered}
$$

We will find a simple solution for $b^{2}$, but we will discover that obtaining a solution for $a$ for vertically-inhomogeneous turbulence, even if stationary, is not a simple task.

On the other hand, the derivation of the Fokker-Planck equation is more complex; van Dop et al., (1985) comment that its derivation "is standard though not quite trivial." This derivation involves three processes and three equations in the following order:

(1) the Markov process and the Chapman-Kolmogorov equation,

(2) the jump process and the master equation, and

(3) the diffusion process and the Fokker-Planck equation.

This sequence of processes and equations is discussed in Wang and Uhlenbeck (1945) Secs. 3, 5, and 8; Doob (1953) Secs. VI.1-3; and van Kampen (1981) Secs. IV.2, V.1, and VIII.1-2. Related material is given in Arnold (1974) and Gardiner (1983). We found the treatment by van Kampen to be the most useful in working from (1) through (3).

As we have noted, in a Markov process "the past and present are statistically independent when the present is known" (Arnold, p. 29). The Chapman-Kolmogorov equation statistically decouples the "future" from the "past" by stating there is a wide range of possible values for the "present" for a given "past" and a given "future." The master equation is a balance equation for jumps into and out of a given state (from the "past" into the "present" and from the "present" into the "future"). The first and second partial derivatives with respect to $w$ in the Fokker-Planck equation, Eq. (8), come from a two-term Taylor series expansion of a special form of the master equation in terms of $w$. The drift coefficient $a$ and the diffusion coefficient $b^{2}$ in Eq. (8) are functions of the first and second moments, respectively, of $d w$ as defined by Eq. (9a-b) for the Langevin equation. This identity of $a$ and $b^{2}$ for the Fokker-Planck and Langevin equations is discussed in Wang and Uhlenbeck (1945) Sec. 9, Doob (1953) Sec. VI.3, van Kampen (1981) Sec. VIII.8, and, at greater length, in Arnold (1974) Secs. 2.5-6 and 9.3-4.

The title of Sec. 4 in Thomson (1987) is "the choice of $\boldsymbol{a}$ and $\boldsymbol{B}$ " where his $\boldsymbol{B}$ is our $\frac{1}{2} b^{2}$. He noted in the first paragraph that $\boldsymbol{a}$ (specifically, a related function $\phi$ ) can be determined uniquely for one dimension but not for two or three. (We consider only the one-dimensional case in this study.) This leaves $\boldsymbol{B}$ to be determined. In the last paragraph, he concluded that it is best to keep the model simple in the absence of sufficient data or a theory to the contrary. He goes on to state that it is simplest to choose $\boldsymbol{B}$ to be independent of $\boldsymbol{u}$ so we will let $b=b(z)$ in Eqs. (7a) and (8). Thomson also proposed that the most rational choice for $\boldsymbol{B}$ is one that we develop in the next paragraph. 
We follow Pope (1983), van Dop et al. (1985), and Thomson (1987) in introducing the Lagrangian structure function, the ensemble average of the square of the change in Lagrangian velocity in the time interval $\Delta t$ (Monin and Yaglom, 1975, pp. 83 and 359):

$$
D(\Delta t)=\left\langle[w(t+\Delta t)-w(t)]^{2}\right\rangle=\left\langle(\Delta w)^{2}\right\rangle .
$$

For Kolmogorov's inertial subrange $\tau_{k} \ll \Delta t \ll \tau$ where $\tau_{k}$ is the Kolmogorov time scale and $\tau$ is the local Lagrangian decorrelation time scale,

$$
D(\Delta t)=C_{0} \varepsilon \Delta t
$$

where $C_{o}$ is a universal constant and $\varepsilon$ is the ensemble-average rate of dissipation of turbulent kinetic energy. The coefficient $b$ in the Langevin equation (Eq. (7a)) and the Fokker-Planck equation (Eq. (8)) can be related to the universal constant $C_{0}$ by taking the ensemble average of the square of Eq. (7a). We assume that $a$ and $b$ are essentially constant during the time interval $\Delta t$ and we apply Eqs. $(5 a-b)$ for the Weiner process. The result is

$$
\left\langle(\Delta w)^{2}\right\rangle=b^{2} \Delta t
$$

Then from Eqs. (10-(12),

$$
b(z)=\left[C_{0} \varepsilon(z)\right]^{\frac{1}{2}}
$$

There has been, and still may be, considerable uncertainty about the value of $C_{0}$. Rodean (1991) reviewed the values that have been published. He also obtained a semi-analytical value of $C_{0}=5.7$ for turbulence in the constant-stress region of the neutral boundary layer. This value is between the values of $C_{0}=2-5$ obtained by Pope and Chen (1990) and $C_{0}=7$ by Sawford (1991).

We use Eq. (13) to rewrite Eqs. (7a) and (8):

$$
\begin{gathered}
d w=a(z, w) d t+\left[C_{0} \varepsilon(z)\right]^{\frac{1}{2}} d W(t), \\
\frac{\partial}{\partial z}(w P)=-\frac{\partial}{\partial w}(a P)+\frac{1}{2}\left[C_{0} \varepsilon(z)\right] \frac{\partial^{2} P}{\partial w^{2}} .
\end{gathered}
$$

Thomson (1987) stated that for Eq. (14) to satisfy the well-mixed condition (if the marked particles of a tracer are well-mixed, they will stay that way), it is necessary and sufficient that $P=P_{a}$ should satisfy Eq. (15) where $P_{a}$ is the probability density function in $z$-w space of the particles of the fluid. This leads to the condition

$$
a(z, w)=\frac{C_{0} \varepsilon(z)}{P_{a}(z, w)} \frac{\partial P_{a}(z, w)}{\partial w}+\frac{\phi(z, w)}{P_{a}(z, w)}
$$

where $\phi$ satisfies

$$
\frac{\partial \phi(z, w)}{\partial w}=-\frac{\partial}{\partial z}\left[w P_{a}(z, w)\right]
$$

and

$$
\phi \rightarrow 0 \text { as }|w| \rightarrow \infty
$$

As we have noted, Thomson stated that $\phi$ is determined uniquely by the well-mixed condition in one dimension, but not in two or three. 
We begin by considering first the most complex solution in $\phi$ : that for the convective boundary layer (CBL) with stationary, non-Gaussian, inhomogeneous turbulence. Baerentsen and Berkowicz (1984) made the valuable contribution of approximating the non-Ciaussian probability density function (PDF) of the CBL with two Gaussian distributions, one for the updrafts and one for the downdrafts:

$$
P_{a}(z, w)=\frac{\lambda_{i}}{\sigma_{i} \sqrt{2 \pi}} \exp \left[-\frac{1}{2}\left(\frac{w-m_{i}}{\sigma_{i}}\right)^{2}\right]
$$

where $i=1,2$ with summation ( $i=1$ for the updrafts and $i=2$ for the down drafts), $\lambda_{1}$ and $\lambda_{2}$ are the respective probabilities of occurrence of updrafts or downdrafts, $m_{i}$ is the mean velocity in the updrafts or downdrafts, and $\sigma_{i}^{2}$ is the variance of the updraft or downdraft velocities $\left(\lambda_{i}\right.$ may also be interpreted as the fraction of the area occupied by the updrafts or downdrafts). The parameters $\lambda_{i}, m_{i}$, and $\sigma_{i}$ are functions of $z$ within the CBL. Baerentsen and Berkowicz equated the zeroth through the third moments of $P_{a}$ to the corresponding moments specified for the PDF of the CBL:

$$
\begin{aligned}
& \lambda_{1}+\lambda_{2}=1, \\
& \lambda_{1} m_{1}+\lambda_{2} m_{2}=0, \\
& \lambda_{1}\left(m_{1}^{2}+\sigma_{1}^{2}\right)+\lambda_{2}\left(m_{2}^{2}+\sigma_{2}^{2}\right)=\sigma_{w}^{2}, \\
& \lambda_{1}\left(m_{1}^{3}+3 m_{1} \sigma_{1}^{2}\right)+\lambda_{2}\left(m_{3}^{3}+3 m_{2} \sigma_{2}^{2}\right)=\left\langle w^{3}\right\rangle .
\end{aligned}
$$

In the above, $m_{2} \leq 0 \leq m_{1}$. We combine Eqs. (18a-b) to get solutions for $\lambda_{1}$ and $\lambda_{2}$ :

$$
\begin{aligned}
& \lambda_{1}=-m_{2}\left(m_{1}-m_{2}\right)^{-1}, \\
& \lambda_{2}=m_{1}\left(m_{1}-m_{2}\right)^{-1} .
\end{aligned}
$$

Weil (1990) introduced the following parameters:

$$
\begin{aligned}
R & =\sigma_{1} / m_{1}=-\sigma_{2} / m_{2} \\
S & =\left\langle w^{3}\right\rangle / \sigma_{w}^{3} \\
\alpha & =\left(1+R^{2}\right)\left(1+3 R^{2}\right)^{-1}, \\
\beta & =1+R^{2}
\end{aligned}
$$

Then from Eqs. (18a)-(20d),

$$
\begin{aligned}
& \frac{m_{1}}{\sigma_{w}}=\frac{1}{2}\left[\alpha S+\left(\alpha^{2} S^{2}+\frac{4}{\beta}\right)^{\frac{1}{2}}\right], \\
& \frac{m_{2}}{\sigma_{w}}=\frac{1}{2}\left[\alpha S-\left(\alpha^{2} S^{2}+\frac{4}{\beta}\right)^{\frac{1}{2}}\right] .
\end{aligned}
$$

Given measured values for $\sigma_{w}$ and $S$ and an assumed value for $R$ (Baerentsen and Berkowicz, as well as others, assumed $R=1$; Weil generalized the solution), $m_{1}, m_{2}, \lambda_{1}$, and $\lambda_{2}$ can be determined.

Luhar and Britter (1989) and Sawford (Weil, 1990) independently derived the solution of Eqs. (14)-(17) for the CBL:

$$
d w=\left(-\frac{C_{0} \varepsilon Q}{P_{a}}+\frac{\phi}{P_{a}}\right) d t+\left(C_{0} \varepsilon\right)^{\frac{1}{2}} d W(t)
$$


Luhar and Brittє: (1989) and Sawford (Weil, 1990) independently derived the solution of Eqs. (14)-(17) for the CBL:

$$
d w=\left(-\frac{C_{0} \varepsilon Q}{P_{a}}+\frac{\phi}{P_{a}}\right) d t+\left(C_{0} \varepsilon\right)^{\frac{1}{2}} d W(t)
$$

where

$$
\phi=-\partial P_{a} / \partial w
$$

The solutions for $Q$ and $\phi$ are

$$
Q=\lambda_{i}\left(w-m_{i}\right) P_{i} / \sigma_{i}^{2}
$$

and

$$
\phi=\sigma_{i}^{2} P_{i} \frac{\partial \lambda_{i}}{\partial z}+\frac{\lambda_{i} P_{i}}{2}\left(1+\frac{w^{2}}{\sigma_{i}^{2}}\right) \frac{\partial \sigma_{i}^{2}}{\partial z}-\frac{1}{2} \operatorname{erf}\left(\frac{w-m_{i}}{\sigma_{i} \sqrt{2 \pi}}\right) \frac{\partial}{\partial z}\left(\lambda_{i} m_{i}\right)
$$

where $i=1,2$ with summation. In Eq. (22a), the first component of $a(z, w) d t$, $-\left(C_{0} \varepsilon Q / P_{a}\right) d t$, is a "fading memory" term and the second component, $\left(\phi / P_{a}\right) d t$, is a "drift." term to account for the vertical inhomogeneity of the turbulence.

The meaning of the last statement becomes more clear for the case of Gaussian inhomogeneous turbulence. As stated by Luhar and Britter and by Weil, Eqs. (22a-d) reduce to the following for Gaussian turbulence:

$$
C_{0} \varepsilon Q / P_{a}=\left(C_{0} \varepsilon / 2 \sigma_{w}^{2}\right) w
$$

and

so

$$
\frac{\phi}{P_{a}}=\frac{1}{2}\left(1+\frac{w^{2}}{\sigma_{w}^{2}}\right) \frac{\partial \sigma_{w}^{2}}{\partial z}
$$

$$
d w=\left[-\left(\frac{C_{0} \varepsilon}{2 \sigma_{w}^{2}}\right) w+\frac{1}{2}\left(1+\frac{w^{2}}{\sigma_{w}^{2}}\right) \frac{\partial \sigma_{w}^{2}}{\partial z}\right] d t+\left(C_{0} \varepsilon\right)^{\frac{1}{2}} d W(t) .
$$

Equation (23b) is the "drift correction" originally proposed by Wilson et al. (1983) who closed their paper with the comment, "Though we can offer no proof that [Eq. (23c)] is 'correct,' it seems worthy of investigation." Thomson (1984) used moment-generating functions to show that, in inhomogeneous turbulence, Eq. (23c) gives Gaussian turbulence (the output) with Gaussian forcing (the input $d W(t)$ ). The generalized unique solution for $\phi / P_{a}$ for one dimension given by Thomson (1987) in his Eq. (30) reduces to Eq. (23b) for stationary turbulence with no mean flow.

The "fading memory" term, Eq. (23a), comes very naturally from the preceding use of the Fokker-Planck equation and the Lagrangian structure function to solve for $a$ and $b$ in Eq. ( $\mathrm{Fa}$ ). The first paper to have used this form appears to be Sawford and Guest (1988) who applied Thomson's (1987) "simplest solution" for $\phi$ in three dimensions to the constant stress region of an idealized neutral boundary layer. The usual form used for many years comes from substituting

$$
\tau=2 \sigma_{w}^{2} / C_{0} \varepsilon
$$

into Eq. (23a) to give $-(w / \tau) d t$ as the "fading memory" term. For inhomogeneous turbulence. $\tau$ is "simply a (rather loosely defined) local decorrelation time scale" (Thomson, 1987). It is only in stationary, homogeneous Gaussian turbulence that Eqs. (23c) and (24a) can be combined to give the classical Langevin equation model,

$$
d w=-\frac{w}{\tau_{L}} d t+\left(\frac{2 \sigma_{w}^{2}}{\tau_{L}}\right)^{1 / 2} d W(t)
$$


The above forms of the Langevin model (Eqs. (22a-d), Eq. (23c), and Eq. (24b)) are applicable to high Reynolds number flows with three-dimensional turbulence for time in the range

$$
\tau_{k} \ll t
$$

where $\tau_{k}$ is the Kolmogorov time scale (Lin and Reid, 1963; Legg and Raupach, 1982; Sawford, 1985; van Dop et al., 1985; Thomson, 1987). $\tau_{k}$ is the time scale for which accelerations remain correlated (see Sawford (1991) for a recent discussion).

In all of the above, the random forcing $d W(t)$ is Gaussian. In the past, Langevin models with non-Gaussian forcing have been proposed for both Gaussian turbulence (Thomson, 1984) and non-Gaussian turbulence (de Baas et al., 1986; Sawford and Guest, 1987), but it has since been established that the random forcing must be Gaussian (Thomson, 1987).

\section{The Random Displacement Model}

The above forms of the Langevin model are used in two steps to determine the random displacement of a marked particle. First, a form of Eq. (7a) is integrated to obtain a solution for the velocity $w(z, t)$ of a marked particle. Second, this solution for $w$ is used in integrating Eq. (7b) to obtain the displacement $z(t)$ for that particle. We now consider the random displacement model with which $z(t)$ is determined directly. Under the condition that the time scale $\tau \rightarrow 0$ ( or $t / \tau \rightarrow \infty$ ), the two equations of the Langevin model, Eqs. (7a-b), can be combined and mathematically transformed into a stochastic differential equation for $z(t)$. This is called the Markov or diffusion equation limit of the Langevin model.

Arnold (1974) pp. 134-136 transformed the Gaussian process for $w(t)$ into a Gaussian process for $z(t)$. Boughton et al. (1987) gave essentially the same transformation in their proposal of a random displacement model for turbulent diffusion in the atmosphere. These transformations are for the equivalent of Eq. (24b), the Langevin model for stationary, homogeneous Gaussian turbulence. Transformations have also been published for Langevin models for stationary inhomogeneous Gaussian turbulence. Durbin (1984) stated that one of the constraints on a consistently formulated Langevin model is that it must reduce to an eddy diffusion model as $\tau \rightarrow 0$ or $t / \tau \rightarrow \infty$. He transformed the equivalent of Eq. (23c) into its diffusion equation limit which we will write as

$$
d z=\frac{\partial}{\partial z}\left(\sigma_{w}^{2} \tau\right) d t+\left(2 \sigma_{w}^{2} \tau\right)^{1 / 2} d W(t)
$$

Equation (23c) meets the "well-mixed" condition and includes the "drift correction" proposed by Wilson et al. (1983). van Dop et al., (1985) presented in their Eq. (48) the Markov limit of a Langevin model that, for stationary, inhomogeneous Gaussian turbulence, incorporates a different "drift correction" proposed by Legg and Raupach (1982). It was subsequently shown that this correction does not meet the "well-mixed" condition. Yet, Eq. (48) in van Dop et al. can be transformed into Eq. (26) exactly. Thomson (1987) showed in his Sec. 3.5 that

(1) A Langevin model that satisfies the well-mixed condition reduces to a diffusion equation as $\tau \rightarrow 0$, and

(2) A Langevin model that reduces to a diffusion equation does not necessarily meet the wellmixed condition. 
(2) A Langevin model that reduces to a diffusion equation does not necessarily meet the wellmixed condition.

de Baas and Troen (1989) demonstrated that the Wilson et al. and Legg and Raupach models reduce to exactly the same Markov limit. We will outline the procedure used by de Baas and Troen (but only for Eq. (23c)) and will indicate an approximation that leads to the same limiting form for the two Langevin models.

Equation (23c) can be rewritten as

$$
d\left(\frac{w}{\sigma_{w}}\right)=\left(-\frac{w}{\sigma_{w} \tau}+\frac{d \sigma_{w}}{d z}\right) d t+\left(\frac{2}{\tau}\right)^{1 / 2} d W(t)
$$

We use the scaling relations

$$
\tau=\alpha \mathrm{T}, \sigma_{w}=\beta \Sigma,
$$

where $T$ and $\Sigma$ are functions of $z$, and integrate the result to get

$$
\alpha\left[\left(\frac{w}{\Sigma}\right)_{t}-\left(\frac{w}{\Sigma}\right)_{0}\right]=\int_{0}^{t}\left[\left(-\frac{w}{\Sigma T}+\alpha \beta^{2} \frac{d \Sigma}{d z}\right) d t+\left(\frac{2 \alpha \beta^{2}}{T}\right)^{1 / 2} d W(t)\right]
$$

Arnold (1974), Boughton et al. (1987) and de Baas and Troen (1989) required the following or its equivalent:

$$
\alpha \rightarrow 0, \beta \rightarrow \infty \text { such that } \alpha \beta^{2}=K \text {, a constant . }
$$

The result is that $\sigma_{w}$ increases less rapidly than $\tau$ decreases as the turbulence field is rescaled. de Baas and Troen stated that these constraints are required in order that the limiting conditions of $\tau \rightarrow 0$ with $t$ fixed and $\tau \rightarrow \infty$ with $\tau$ fixed be equivalent. The term on the left in Eq. (29a) is, under these conditions, bounded in the "mean square" sense for all time. We recall Eq. (7b),

$$
d z=w d t
$$

We combine Eqs. $(29 a-c)$ to get

$$
\int_{z_{0}}^{z_{t}} \frac{d z}{\Sigma T}=\int_{0}^{t}\left[K \frac{d \Sigma}{d z} d t+\left(\frac{2 K}{T}\right)^{1 / 2} d W(t)\right]
$$

We convert Eq. (30) to a stochastic differential equation by differentiating both sides. We use the rules of the Ito calculus (Arnold, pp. 89-92) to differentiate the left side. This involves the use of the first two terms of the Taylor's series

$$
f(x)-f(a)=(x-a)) f^{\prime}(a)+\frac{(x-a)^{2}}{2 !} f^{\prime \prime}(a)+\ldots,
$$

where $(x-a)$ corresponds to $d z$ and $f^{\prime}(a)$ corresponds to $(\Sigma T)^{-1}$. Then the differential of Eq. (30) is

$$
\frac{d z}{\Sigma T}-\frac{1}{2}\left(\frac{d z}{\Sigma T}\right)^{2} \frac{d}{d z}(\Sigma T)=K \frac{d \Sigma}{d z} d t+\left(\frac{2 K}{T}\right)^{1 / 2} d W(t)
$$


This is of the form

$$
A(d z)^{2}+B d z+C=0
$$

With $B=-1$, we select the root

$$
d z=\left[1-(1-4 A C)^{1 / 2}\right] / 2 A .
$$

We expand $(1-4 A C)^{1 / 2}$ in the infinite series

$$
(1-4 A C)^{1 / 2}=1-\frac{4 A C}{2}-\frac{(4 A C)^{2}}{8}-\ldots
$$

and retain only the first three terms. This is in accord with the rules of the Ito calculus that we retain terms involving $(d W(t))^{2}$ because

$$
\begin{aligned}
(d t)^{2} & =0 \\
d W(t) d t & =0 \\
(d W(t))^{2} & =d t
\end{aligned}
$$

The final result is

$$
d z=\frac{d}{d z}\left(\sigma_{w}^{2} \tau\right) d t+\left(2 \sigma_{w}^{2} \tau\right)^{1 / 2} d W(t)
$$

which is identical to Eq. (26). The first term on the right is analogous to the "drift correction" given by Eq. (23b).

We have stated that our Lagrangian model, Eq. (23c), and another with a different "drift correction" have Eqs. (26) and (38) as a common Markov limit. Inspection of the transformations of each Langevin model in de Baas and Troen (1989) shows that the difference between these models disappears as a consequence of the approximation of using only the first three terms of the infinite series given by Eq. (36).

The Fokker-Plank equation corresponding to Eq. (38) is

$$
\frac{\partial}{\partial z}\left\{\left[\frac{\partial}{\partial z}\left(\sigma_{w}^{2} \tau\right)\right] P_{a}(z)\right\}=\frac{1}{2} \frac{\partial^{2}}{\partial z^{2}}\left[\left(2 \sigma_{w}^{2} \tau\right) P_{a}(z)\right]=0
$$

These are the two equivalent forms of the random displacement model for vertical diffusion in stationary, inhomogeneous Gaussian turbulence. This model is applicable to a different time range than that given by Eq. (25) for the Langevin model:

$$
\tau \ll t
$$

(See Eqs. (10)-(11) and related discussion concerning the relation between $\tau_{k}$ and $\tau$.)

We have not discussed transforming the Langevin model for the CBL, Eqs. (7b) and (22a-d) into the random displacement model. The reasons why we have not will be discussed in the next section. 


\section{The Eddy Diffusivity Model}

In the preceding section, the random displacement model has been described as the Markov or diffusion equation limit of the Langevin model. This implies, correctly, that there is a close relation between the random displacement model and the eddy diffusivity model. From Monin and Yaglom (1971) Sec. 10.3 and p. 615 ,

$$
K=K(z)=\sigma_{w}^{2} \tau
$$

where $K$ is the eddy diffusivity. Then the stochastic differential equation for the random displacement process, Eq. (38), becomes

$$
d z=\frac{d K}{d z} d t+(2 K)^{1 / 2} d W(t)
$$

and the correspouding Fokker-Planck equation, Eq. (39), is

$$
\frac{\partial}{\partial z}\left[\frac{\partial K}{\partial z} P_{a}(z)\right]-\frac{1}{2} \frac{\partial^{2}}{\partial z^{2}}\left[2 K P_{a}(z)\right]=0
$$

This reduces to

$$
\frac{\partial}{\partial z}\left[K \frac{\partial P_{a}(z)}{\partial z}\right]=0
$$

which has the same form as the diffusion equation, Eq. (3), which, for stationary turbulence and mean zero flow in the $\mathrm{z}$-direction, takes the form

$$
\frac{\partial}{\partial z}\left(K_{\theta} \frac{\partial \Theta}{\partial z}\right)=0
$$

The equivalence of Eqs. (44) and (45) by means of a transformation from $P$ to $\Theta$ is discussed by Boughton et al. (1987). The time constraints of Eq. (40) apply to Eqs. (41)-(45) as well as to Eqs. (38) and (39) (Monin and Yaglom, 1971, Sec. 10.31. (This restriction is avoided by letting $K$ be an unphysical function of time and/or distance from the point of scalar release.)

At the end of the preceding section, we remarked that we had not discussed transformation of the Langevin model for the CBL into the random displacement model but that we would do so in this section. Our reason was that this discussion is more clear using terminology associated with the diffusion equation.

In a physical system for which the diffusion equation, Eq. (3),

$$
\frac{\partial \Theta}{\partial t}+U_{i} \frac{\partial \Theta}{\partial x_{i}}=\frac{\partial}{\partial x_{i}}\left(K \frac{\partial \Theta}{\partial x_{i}}\right)
$$

is valid (here we write the general form), the diffusivity $K$ is a positive quantity, just as are $\sigma_{w}^{2}$ and $\tau$ in Eq. (41). It has been known for many years that the eddy diffusivity model is not applicable everywhere in the CBL. Furthermore, the applicability or non-applicability is a function of the location of the source of the scalar quantity released into the CBL. For a source at the top of the $\mathrm{CBL}$, the effective eddy diffusivity $K_{\text {eff }}$ is well-behaved for downward diffusive transport. On the other hand, the eddy diffusivity model fails for a source at the bottom. About halfway up, there is a singularity in the value of $K_{\text {eff }}$ (from $K_{\text {eff }} \gg 0$ to $K_{\text {eff }} \ll 0$ ) and there is a counter-gradient flux (meaning negative $K_{\text {eff }}$ ) in the upper part of the CBL. This failure of the eddy diffusivity 
model (and the equivalent random displacement model) was the motivation of Baerentsen and Berkowicz (1984), de Baas et al. (1986), Sawford and Guest (1987), Luhar and Britter (1989), and Weil (1990) for developing various Langevin models for the CBL. There have also been efforts to develop corrections for this eddy diffusivity model (e.g., Holtslag and Moeng, 1991). In addition, there have been studies to determine the reason for the failure of the diffusion equation (without corrections) to model the asymmetric vertical transport of scalars within the CBL. Wyngaard (1987) proposed that the skewness of the turbulence is responsible for the observed phenomena. Wyngaard and Weil (1991) concluded that this asymmetry is a consequence of the interaction of the turbulence skewness $\left\langle w^{3}\right\rangle / \sigma_{w}^{3}$ and the scalar flux gradient $\partial<\theta w>/ \partial z$. Therefore, neither the eddy diffusivity model nor the random displacement model are generally applicable, without modification, to non-Gaussian turbulence.

It is possible that the Langevin model for the CBL has a diffusion limit. However, this limit may not be physically realistic because of the requirement that $K>0$.

\section{Model Inputs}

The input parameters for the Langevin models we have considered include

(1) the variance of the vertical velocity $w, \sigma_{w}^{2}$, and

(2) the rate of dissipation of turbulence kinetic energy $\varepsilon$.

An alternate to $\varepsilon$ (by means of Eq. $\left.(24 \mathrm{a}), \tau=2 \sigma_{\omega}^{2} / C_{0} \varepsilon\right)$ is

(3) the local decorrelation time scale $\tau$

which is equal to the Lagrangian integral time scale $\tau_{L}$ in homogeneous turbulence. The parameters $\sigma_{\omega}^{2}, \varepsilon$, and $\tau$ are generally functions of both $z$ and the atmospheric stability; the effects of atmospheric stability on these parameters will be discussed in a later part of this study. For the convective boundary layer, we also need as function of $z$

(4) the relative probabilities of updrafts and downdrafts $\lambda_{i}$,

(5) the mean velocities in updrafts and downdrafts $m_{i}$, and

(6) the skewness $\left\langle w^{3}\right\rangle / \sigma_{w}^{3}$.

The first three of the above ( $\sigma_{w}^{2}$ and $\varepsilon$ or $\tau$ ) also serve as inputs to the random displacement model. They also serve as inputs to the eddy diffusivity model by means of the relation from Eqs. (24a) and (41),

$$
K=\sigma_{w}^{2} \tau=2 \sigma_{w}^{4} / C_{0} \varepsilon
$$

\section{Applicability of Each Model}

Of the three models we have studied, the Langevin model is the most generally applicable because it can be used to model diffusion in both Gaussian and non-Gaussian turbulence for all times greater than the Kolmogorov time scale $\tau_{k}$,

$$
\tau_{k} \ll t
$$


provided that the turbulence is three-dimensional and at very high Reynolds number. It is a consequence of the limiting conditions ( $\tau \rightarrow 0$ with $t$ fixed and $t \rightarrow \infty$ with $\tau$ fixed) imposed in transforming the Langevin model into the random displacement model that the time for which the latter model is applicable is

$$
\tau \ll t
$$

The above two relations indicate that, for the release of "marked particles" from a point source, it is appropriate to begin the simulation with the Langevin model and switch to the random displacement model at some time $t \gg \tau$. Similarly, the use of the eddy diffusivity model, in which the eddy diffusivity is a function of only the turbulence statistics, is not in order until $t \gg \tau$. In practical situations involving complex flows with inhomogeneous and nonstationary turbulence, the condition imposed by Eq. (49) may not be achieved.

We have briefly discussed the failure of the eddy diffusivity model to simulate scalar turbulent diffusion throughout the convective boundary layer, bottom-up and top-down. Recent investigations indicate that the skewness of the turbulence in the CBL is a key factor in this failure. Inhomogeneity is also important. Therefore the eddy diffusivity model (and the equivalent random displacement model) should be used with caution in the CBL and other flows with non-Gaussian turbulence.

\section{References}

Arnold, L., 1974: Stochastic differential equations: Theory and applications. John Wiley and Sons, New York.

Baerentsen, J.H., and R. Berkowicz, 1984: Monte Carlo simulation of plume dispersion in the convective boundary layer. Atmos. Environ., 18, 701-712.

Boughton, B.A., J.M. Delaurentis, and W.E. Dunn, 1987: A stochastic model of particle dispersion in the atmosphere. Boundary-Layer Meteorol., 40, 147-163. 1989: 48, 443-444.

Chandrasekhar, S., 1943: Stochastic problems in physics and astronomy. Rev. Mod. Phys., 15, $1-89$.

de Baas, A.F., and I. Troen, 1989: A stochastic equation for diffusion in inhomogeneous conditions. Physica Scripta, 40, 64-72.

de Baas, A.F., H. van Dop, and F.T.M. Nieuwstadt, 1986: An application of the Langevin equation for inhomogeneous conditions to dispersion in the convective boundary layer. Quart. J.R. Met. Soc., 112, 165-180.

Doob, J.L., 1953: Stochastic processes. John Wiley and Sons, New York.

Durbin, P.A., 1984: Comments on Paper by Wilson et al. (1981) and Legg and Raupach (1982). Boundary-Layer Meteorol., 29, 409-411.

Gardiner, C.W., 1983: Handbook of stochastic methods. Springer-Verlag, Berlin.

Holtslag, A.A.M., and C.-H Moeng, 1991: Eddy diffusivity and counter gradient transport in the convective atmospheric boundary layer. J. Atmos. Science, 48, 1690-1698. 
Legg, B.J., and M.R. Raupach, 1982: Markov-chain simulation of particle dispersion in inhomogeneous flows: The mean drift velocity induced by a gradient in Eulerian velocity variance. Boundary-Layer Meteorol., 24, 3-13.

Lin, C.C., and W.H. Reid, 1963: Turbulent flow, theoretical aspects. Handbuch der Physik, VIII/2, $438-523$.

Luhar, A.K., and R.E. Britter, 1989: A random walk model for dispersion in inhomogeneous turbulence in a convective boundary layer. Atmos. Environ., 23, 1911-1924.

Monin, A.S., and A.M. Yaglom; 1971, 1975: Statistical fluid mechanics: Mechanics of turbulence, Vols. 1 and 2. MIT Press, Cambridge.

Obukhov, A.M., 1959: Description of turbulence in terms of Lagrangian variables. Adv. Geophys., 6, 113-116.

Pope, S.B., 1983: A Lagrangian two-time probability density function equation for inhomogeneous turbulent flows. Phys. Fluids, 26, 3448-3450.

Pope, S.B., and Y.L. Chen, 1990: The velocity-dissipation probability density function model for turbulent flows. Phys. Fluids, 42, 1437-1449.

Rodean, H.C., 1991: The universal constant for the Lagrangian structure function. Phys. Fluids, A3, 1479-1480.

Sawford, B.L., 1985: Lagrangian statistical simulation of concentration mean and fluctuation fields. J. Climate Appl. Meteorol., 24, 1152-1166.

Sawford, B.L., 1991: Reynold number effects in Lagrangian stochastic models of turbulent dispersion. Phys. Fluids, A3, 1577-1586.

Sawford, B.L., and F.M. Guest, 1987: Lagrangian stochastic analysis of flux-gradient relationships in the convective boundary layer. J. Atmos. Sci., 44, 1152-1165.

Sawford, B.L., and F.M. Guest, 1988: Uniqueness and universality of Lagrangian stochastic models of turbulent dispersion. Proceedings of the 8th Symp. on Turbulence and Diffusion (Amer. Meteorol. Soc., Boston) 96-99.

Thomson, D.J., 1984: Random walk modeling in diffusion in inhomogeneous turbulence. Quart. J.R. Met. Soc., 110, 1107-1120.

Thomson, D.J., 1987: Criteria for the selection of stochastic models of particle trajectories in turbulent flows. J. Fluid Mech., 180, 529-556.

van Dop, H., F.T.M. Nieuwstadt, and J.C.R. Hunt, 1985: Random walk models for particle displacements in inhomogeneous unsteady turbulent flows. Phys. Fluids, 28, 1639-1653.

van Kampen, N.G., 1981: Stochastic processes in physics and chemistry. North-Holland Publishing Company, Amsterdam.

Wang, M.C., and G.E. Uhlenbeck, 1945: On the theory of the Brownian motion II. Rev. Mod. Phys., 17, 323-342.

Weil, J.C., 1990: A diagnosis of the asymmetry in top-down and botton-up diffusion using a Lagrangian stochastic model. J. Atmos. Sci., 47, 501-515. 
Wilson, J.D., B.J. Legg, and D.J. Thomson, 1983: Calculation of particle trajectories in the presence of a gradient in turbulent-velocity variance. Boundary-Layer Meteorol., 27, 163-169.

Wyngaard, J.C., and J.C. Weil, 1991: Transport asymmetry in skewed turbulence. Phys. Fluids, A3, 155-162.

Wyngaard, J.C., 1987: A physical mechanism for the asymmetry in top-down and bottom-up diffusion. J. Atmos. Science, 44, 1083-1087.

Langevin Notes, Part I.tex 


\title{
Part II: Studies of the Langevin Model Applications, and the Theory of Stochastic Processes
}

\begin{abstract}
The history of the Langevin equation from its beginning in 1908 as a model for Brownian motion to its recent applications as a model for the turbulent diffusion of scalars in the atmospheric boundary layer is summarized. "Drift corrections" for inhomogeneous turbulence are studied, beginning with two made on an ad hoc basis and ending with a modification of one that has a sound mathematical basis. A detailed exarnination is made of some of the mathematics unique to stochastic processes, including "white noise" and the related Wiener process, realizable probability distributions, the Markov or diffusion equation limit of the Langevin equation, the derivation of the Fokker-Planck equation and the relation between the Lagrangian Langevin equation and the Eulerian Fokker-Planck equation. Application of the Langevin model to the atmospheric boundary layer, boundary conditions, and parameterizations of turbulence statistics for use as model inputs are reviewed. Available experimental diffusion data sets and carididate test problems for checking the performance of the Langevin model are surveyed.
\end{abstract}

\section{Introduction}

"Traditional Eulerian methods of modeling turbulent dispersion use ... approximate relations ... [that] are not uniformly valid.... The assumptions made in Lagrangian modeling ... do not depend on the concentration field or the source distribution and, in this sense, are uniformly valid" (Sawford, 1985).

"Stochastic or random-walk models ... are particularly suited to the calculations in complex flows where many other techniques are inappropriate or invalid" (Thomson, 1987).

These quotations explain the motivation for this study of a stochastic Lagrangian diffusion model based on the Langevin equation. The following is not a comprehensive review but a selective description of the sequence of the developments of greatest interest.

\section{Brownian Motion}

The following historical survey is based on Chandrasekhar (1943), van Kampen (1981), and Gardiner (1983).

Our story begins in 1827 when Robert Brown studied what became known as Brownian motion: Tiny pollen grains suspended in water exhibited very agitated and irregular motion. Albert Einstein published the first satisfactory explanation of this phenomenon in 1905; this work is considered to mark the beginning of stochastic modeling of natural phenomena. P. Langevin published an alternate solution for Brownian motion in 1908. He introduced the Lagrangian equation for particle motion that is named after him. We will write it in the form

$$
d u / a t=-\alpha u+\beta \xi(t)
$$


where $u$ is the particle velocity, $\alpha$ is a damping coefficient associated with viscous drag on the particle, and the product of the coefficient $\beta$ and the random function $\xi(t)$ is a rapidly fluctuating acceleration component from irregular and unsymmetrical molecular bombardment on the particle. Langevin's equation was the first example of a stochastic differential equation, but an adequate mathematical basis for this equation was not available until more than 40 years later when Ito formulated his concepts for stochastic differential equations. As Chandrasekhar (1943) remarked, "...'solving' a stoch 1 stic differential equation like [the above] is not the same thing as solving any ordinary differential equation." This is because $\xi(t)$ has only statistically defined properties.

The Eulerian equivalent to the Langevin equation is a partial differential equation, the FokkerPlanck equation. It is named after A.D. Fokker who published a paper on Brownian motion in 1914 and M. Planck who published a more general discussion in 1917.

\section{Turbulent Diffusion}

Taylor (1921) was the first to apply the statistics of Lagrangian particle motion to the problem of turbulent diffusion. He did not make any explicit references to the earlier work of Einstein, Langevin, and others in Brownian motion, but he noted some similarities between turbulent diffusion and "the drunkard's walk." He introduced the velocity autocorrelation function and obtained two fundamental results: the mean displacement in turbulent diffusion is initially proportional to time and, at late times, is proportional to the square root of time.

Our special interest in the Langevin and Fokker-Planck equations has its origin in Obukhov's (1959) suggestion that the Fokker-Planck equation be used to model turbulent diffusion. His suggestion was met with some skepticism as shown by the published discussion of his paper. But Obukhov's proposal led to a new line of development for turbulent diffusion models. Most workers did not follow Obukhov's idea of using the Fokker-Planck equation but, implicitly if not explicitly, used the Langevin equation. The first theoretical papers on this subject were published in the 1960s, the first numerical simulations appeared in the 1970s, and there have been significant advances in both theory and applications since 1980 .

The first to take up Obukhov's idea was Lin who published two papers in 1960 that were incorporated into Ch. 7 of Lin and Reid (1963). Novikov (1963) was next. Krasnoff and Peskin (1971) followed with what is largely a critique of Lin's model.

Hanna (1979) did not mention any of the above in showing that a finite-difference equivalent of the Langevin equation is approximately valid for Lagrangian and Eulerian wind speed observations in the atmospheric boundary layer. Gifford (1982) cited Hanna's results as well as the above authors in proposing that the Langevin equation be used to model horizontal diffusion in the atmosphere over diffusion times ranging from seconds to days. Gifford's proposal was criticized by Sawford (1984) who noted that the Langevin equation is applicable to three-dimensional turbulence whereas relative diffusion on a global scale is quasi-two-dimensional.

Early numerical simulations using the above Langevin equation, Eq. (1), did not give physically realistic results in nonuniform turbulence fields. Specifically, particle concentrations tended to increase in regions with low velocity variance in violation of the second law of thermodynamics. Wilson, Thurtell, and Kidd (1981) were among the first to propose the addition of a drift correction term as a corrective measure. Legg and Raupach (1982) followed with an alternate proposal. 
Both groups used physical reasoning, but each correction was an ad hoc addition to the Langevin equation. Despite the differences between these proposals, they gave similar results in systems with small gradients of velocity variance. Wilson from one team and Legg from the other joined with Thomson to show why the two models could, under certain conditions, give similar solutions. They also demonstrated that a modification of the Wilson et al. (1981) model gave the best results of all in inhomogeneous turbulence (Wilson, Legg, and Thomson, 1983). They remarked in closing. "Though we can offer no proof that [the third model] is 'correct,' it seems worthy of investigation."

Thomson (1984) carried on and used moment-generating functions for the probability densities of the velocity fluctuations $u$ and the random function $\xi$ to derive the model of Wilson et al. (1983). (Moment-generating functions are integral transforms and are related to characteristic functions which, in turn, are a special application of the Fourier transform.) van Dop, Nieuwstadt, and Hunt (1985) transformed the Langevin equation into its Eulerian equivalent, the Fokker-Planck equation, and determined the coefficients in the Langevin equation by relating the random walk model to the Eulerian conservation equations for mass and species. Sawford (1986) showed that their results were equivalent to those of Thomson and demonstrated that "... only the Wilson et al. (1983) model can be realized to satisfy the second law of thermodynamics for Gaussian inhomogeneous turbulence." This model can be written in incremental form as

$$
d\left(\frac{w}{\sigma_{w}}\right)=-\left(\frac{w}{\sigma_{w}}\right) \frac{d t}{\tau_{L}}+\left(\frac{2}{\tau_{L}}\right)^{\frac{1}{2}} \xi(t) d t+\frac{d \sigma_{w}}{d z} d t
$$

where $w$ is the vertical velocity fluctuation, $\sigma_{w}^{2}$ is the variance of $w$, and $z$ is the vertical coordinate. In addition, $\tau_{L}$ is the Lagrangian timescale for the velocity autocorrelation, $\xi(t)$ is a random Gaussian function, and the last term on the right is a "drift correction."

\section{The Langevin Equation for Turbulent Diffusion}

We begin with a reinterpretation of the physical meaning of the Langevin equation: as a model for turbulent diffusion. Next we use a classic solution for Brownian motion to define the coefficients $\alpha$ and $\beta$ [see Eq. (1)] in terms of two properties of stationary, homogeneous turbulence. Then we consider some aspects of the modern theory of stochastic differential equations developed by Ito. We conclude with the results of Thomson $(1984,1987)$, van Dop et al. (1985), and Sawford (1986), concerning the model proposed for inhomogeneous turbulence [see Eq. (2)] by Wilson et al. (1983).

\section{Physical Reinterpretation}

The classic form of the Langevin equation is that of Eq. (1):

$$
d u / d t=-\alpha u+\beta \xi(t)
$$

although some workers use $\beta(t)$ instead of $\beta \xi(t)$. We will find that there are advantages to $\beta \xi(t)$. For use in turbulence, $u$ is the Lagrangian velocity fluctuation from the mean of a "marked" particle of either the fluid or a neutrally, buoyant particle of a different material. The coefficient $\alpha$ is not related to fluid viscosity for Brownian motion, but is a measure of the "fading memory" of the velocity at an earlier time. The product of the coefficient $\beta$ and the random function $\xi(t)$ does 
not represent irregular acceleration from molecular bombardment as in Brownian motion. Rather it represents random acceleration from pressure forces with short correlation times on the order of the Kolmogorov's time scale $\tau_{k}$ (Lin and Reid, 1963; Monin and Yaglom, 1975, pp. 370 and 548549; Sawford, 1985). As Chandrasekhar (1943) remarked about Eq. (3) as applied to Brownian motion,... we should draw attention... to the very drastic nature of assumptions implicit in the very writing of an equation of the form of [Eq. (3)]. For we have in reality supposed that we can divide the phenomenon into two parts, one in which the discontinuity of the events taking place is essential while in the other it is trivial and can be ignored."

The above is consistent with Kolmogorov's (1941) theory of the local structure of turbulence at very large Reynolds numbers: the viscous forces are not significant relative to the pressure forces except at very small length, velocity, and time scales associated with his name. From Monin and Yaglom (1975) pp. 200 and 348, Kolmogorov's scales are

$$
\begin{aligned}
\eta & =\left(\nu^{3} / \varepsilon\right)^{\frac{1}{4}}, \\
v & =(\nu \varepsilon)^{\frac{1}{4}}, \\
\tau_{k} & =\eta / v=(\nu / \varepsilon)^{\frac{1}{2}}
\end{aligned}
$$

for length, velocity, and time, respectively. Here $\varepsilon$ is the rate of turbulent kinetic energy dissipation and $\nu$ is the kinematic viscosity. The Reynolds number based on the above length and velocity scales is

$$
R e=\eta v / \nu=1
$$

The inertial forces are therefore equal to the viscous forces at this scale. Sawford (1984, 1985) states that the Langevin equation is applicable to high Reynolds number, three-dimensional turbulence for time $t \gg \tau_{k}$.

\section{Diffusion in Stationary Homogeneous Turbulence}

Equation (3) is a stochastic differential equation, but it is possible to get some results by means of the conventional method for an ordinary, first-order, linear differential equation of the form

$$
d y / d x+P y=Q
$$

where $P$ and $Q$ are independent of $y$ but may be functions of $x$. The following solution was obtained by Uhlenbeck and Ornstein (1930) for Brownian motion and is given, with variations in Arnold (1974) pp. 134-136, van Kampen (1981) pp. 237-239, and (for turbulent diffusion) Legg and Raupach (1982). We assume, as they did, that $\alpha$ and $\beta$ are constants so the solution of Eq. (3) is

$$
u(t)=u(0) e^{-\alpha t}+\beta e^{-\alpha t} \int_{o}^{t} e^{\alpha s} \xi(s) d s .
$$

Before we can proceed, we need to specify some statistical properties of the velocity fluctuation $u(t)$ and the random function $\xi(t)$. The ensemble average of $u(t)$ is

$$
\langle u(t)\rangle=0 .
$$


The fluctuation $u(t)$ and the function $\xi(t)$ are uncorrelated so

$$
\langle u(t) \xi(t)\rangle=0 .
$$

The ensemble average of $\xi(t)$ is

$$
\langle\xi(t)\rangle=0
$$

and its covariance for times $s$ and $t$ is

$$
\langle\xi(s) \xi(t)\rangle=\delta(t-s)
$$

where $\delta$ is the Dirac delta function with dimension $t^{-1}$. Uhlenbeck and Ornstein were not as drastic in their equivalent to Eq. ( $8 \mathrm{~d})$; they required that $\xi(s)$ and $\xi(t)$ be correlated only if $|t-s|$ is very smail, consistent with the physics of Brownian motion. For our application to turbulence, $\xi(s)$ and $\xi(t)$ are correlated only if $|t-s| \approx \tau_{k}$, the Kolmogorov time scale. However, it is more convenient mathematically to let $\delta$ be the Dirac delta function.

To continue, we square Eq. (7), take the ensemble average of the result, and apply Eq. (8b) to get

$$
\left\langle u^{2}(t)\right\rangle=\left\langle u^{2}(0)\right\rangle e^{-2 \alpha t}+\beta^{2} e^{-2 \alpha t} \int_{0}^{t} \int_{0}^{s} e^{\alpha(r+s)}\langle\xi(r) \xi(s)\rangle d r d s .
$$

We simplify the above with the use of Eq. (8d) to get

$$
\left\langle u^{2}(t)\right\rangle=\left\langle u^{2}(0)\right\rangle e^{-2 \alpha t}+\beta^{2} \int_{0}^{t} e^{2 \alpha(s-t)} d s .
$$

We get the following by evaluating the integral:

$$
\left\langle u^{2}(t)\right\rangle=\left\langle u^{2}(0)\right\rangle e^{-2 \alpha t}+\frac{\beta^{2}}{2 \alpha}\left(1-e^{-2 \alpha t}\right) .
$$

If we assume that the turbulence is stationary and homogeneous, the variance of $u^{2}$ is

$$
\sigma_{u}^{2}=\left\langle u^{2}(0)\right\rangle=\left\langle u^{2}(t)\right\rangle
$$

then Eq. (11) and (12) combine to give

$$
\beta=\sigma_{u} \sqrt{2 \alpha}
$$

With respect to Eqs. (3) and (8d), $\beta^{2}$ is numerically (but not dimensionally) equal to the variance of the random component of the acceleration.

In Brownian motion, Eq. (13) represents the equilibrium between the random motion of the particles and the damping of that motion by fluid viscosity. In turbulence, it represents an equilibrium between correlation and decorrelation of the Lagrangian particle velocity. The Lagrangian velocity correlation coefficient is

$$
R_{L}(t)=\langle u(0) u(t)\rangle /\left\langle u^{2}(0)\right\rangle
$$


and the integral times cale of the Lagrangian velocity is

$$
\tau_{L}=\int_{0}^{\infty} R_{L}(t) d t
$$

We complete our solution of Eq. (3) by multiplying Eq. (7) by $u(0)$, taking the ensemble average of the result, and applying Eq. (8b) to get

$$
\langle u(0) u(t)\rangle=\left\langle u^{2}(0)\right\rangle e^{-\alpha t} .
$$

Equations (14)-(16) together give

$$
R_{L}(t)=e^{-\alpha t}
$$

and

$$
\tau_{L}=\alpha^{-1}
$$

Then we can rewrite Eq. (3) as

$$
d u=-\left(u / \tau_{L}\right) d t+\sigma_{u}\left(2 / \tau_{L}\right)^{\frac{1}{2}} \xi(t) d t .
$$

With $u$ and $\sigma_{u}$ in meters per second $\left(m s^{-1}\right)$ and $\tau_{L}$ in seconds $(s)$, it follows that the unit of $\xi(t)$ is $s^{-\frac{i}{2}}$, another strange property of this random function. We will examine further the properties of $\xi(t)$ in the next section.

\section{From "White Noise" to the "Wiener Process"}

We have stated in Eqs. ( $8 c-d)$ that the ensemble average of the random function $\xi(t)$ is zero and its covariance for times $s$ and $t$ is the Dirac delta function. These properties are noted in the Introduction of Arnold (1974) pp. xi-xii where $\xi(t)$ is also described as "white noise," a stationary Gaussian process with a constant spectral density on the real frequency axis. Arnold adds that $\xi(t)$ is everywhere discontinuous and that its integral is a continuous, but not a differentiable, proce: : He makes it clear that this process does not exist in the physical sense but is a very useful mathematical idealization.

The name "white noise" is given to $\xi(t)$ because it has a uniform spectral density, a characteristic of the Dirac delta function. The Fourier transform of the covariance of $\xi(t)$, given by Eq. (8d), is

$$
F(w)=\int_{-\infty}^{\infty} e^{i w t} \delta(t) d t=1
$$

where $i=\sqrt{-1}$ (Arnold, 1974, pp. 50-51; Papoulis, 1962, pp. 36-37). G- -iner (1983) also discusses white noise and shows that the shorter the correlation tirne, the flatter the spectrum. van Kampen (1981) pp. 237-238 and 241-242 identifies the above properties of $\xi(t)$ as white noise and states, "Thus $[\xi(t)]$ may be visualized as a dense succession of small positive and negative pulses." The amplitudes of these pulses are such that they fit a Gaussian distribution.

We now introduce the "Wiener process," the continuous but not differentiable integral of $\xi(t)$ as defined above. It is interesting to note the sequence with which Arnold and Gardiner treat white noise and the Wiener process. Arnold discusses first white noise and then the Wiener process in 
the Introduction (pp. xi-xiv) but reverses the sequence in Ch. 3 (pp. 45-56). Similarly, Gardiner mentions white noise toward the end of Ch. 1 (pp. 19-20) but describes the Wiener process in Ch. 3 (pp. 66-70) and white noise in the beginning of Ch. 4 (pp. 80-83) before going back to the Wiener process. They emphasize the Wiener process because it, as a continuous function, is superior to discontinuous white noise for use in integrating stochastic differential equations. They show that $\xi(t)$ as white noise is related as follows to the Wiener process $W(t)$ :

$$
W(t)=\int_{0}^{t} \xi(s) d s
$$

As Arnold (pp. xil and 53) indicates, we can write the above symbolically as

$$
d W(t)=\xi(t) d t
$$

We note that $d W(t) / d t=\xi(t)$ is meaningless because $\xi(t)$ is everywhere discontinuous. Therefore $W(t)$ is not differentiable.

We use Eq. (21b) to transform Eq. (19) into the form of the Langevin equation used by Sawford (1984, 1985), van Dop et al. (1985), and Thomson (1987):

$$
d u=-\left(u / \tau_{L}\right) d t+\sigma_{u}\left(2 / \tau_{L}\right)^{\frac{1}{2}} d W(t) .
$$

We noted in the comment following Eq. (19) that the dimension of $\xi(t)$ is $s^{-\frac{1}{2}}$. Here the dimension of $d W(t)$ is $s^{\frac{1}{2}}$. Sawford and van Dop et al., state that

$$
\langle d W(t)\rangle=0
$$

which is consistent with Eq. $(8 c)$ for $\xi(t)$. They also state that

$$
\left\langle[d W(t)]^{2}\right\rangle=d t
$$

which is consistent with the dimension of $d W(t)$ in Eq. (22), but we need further explanation for the quantitative relation expressed in Eq. (23b).

The following on the Wiener process is based largely on Arnold and Gardiner. Arnold (p. 45) calls this process "... a mathematical model of Brownian motion of a free particle with friction neglected," and Gardiner also mentions its applicability to Brownian motion. It is a Gaussian stochastic process with continuous (but not differentiable) sample functions. It is a Markov process in which "... the past and future are statistically independent when the present is known," (Arnold. p. 29). van Dop et al. (1985) observe that "A stochastic process is Markovian when its future state depends only on its present state (and some transition rule)." For a one-dimensional Wiener process, this transformation rule is given by the following probability density function for $W(t)$ :

$$
p\left(w, t \mid w_{o}, t_{o}\right)=\left[2 \pi\left(t-t_{o}\right)\right]^{-\frac{1}{2}} \exp \left[-\left(w-w_{o}\right)^{2} / 2\left(t-t_{o}\right)\right]
$$

where $w_{o}$ is both an initial and a mean value of $W(t), t_{o}$ is the initial time, and $\left(t-t_{o}\right)^{2}$ is the variance of $w$. If $w_{o}=0$,

$$
\langle W(t)\rangle=0
$$


and

$$
\left\langle|w(t)|^{2}\right\rangle=t-t_{0}
$$

If we let $\left(w-w_{o}\right) \rightarrow \Delta w$ with $w_{o}=0$, Eqs. $(25 \mathrm{a}-\mathrm{b}) \rightarrow$ Eqs. $(23 \mathrm{a}-\mathrm{b})$. Arnold (p. 46) states and Gardiner (pp. 69-70) demonstrates that the increments of $W(t), d W(t)$, have a Gaussian distribution and that these increments are independent of each other and the initial value of $W(t)$. Gardiner adds, "The aspect of having independent increments $\Delta W_{i}$ is very important in the definition of stochastic integration ...."

The preceding on white noise $\xi(\mathrm{t})$ and the Wiener process $W(t)$ is almost entirely from the mathematical literature. Now let us review key papers in Lagrangian modeling of turbulent diffusion with respect to the properties of $\xi(\mathrm{t})$ and $W(t)$ and citation of the mathematical literature. If there is no mention of a citation in the following, there was none. Obukhov (1959) assumed that "The evolution of the state of the selected particle in time forms a Markov process" and used the FokkerPlanck instead of the Langevin equation. The Langevin equation was implicitly, if not explicitly, used by the following modelers of turbulent diffusion. Lin and Reid (1963) referred to $\xi(t)$ as a stationary random process. Novikov (1963) called the asympotic form of the spectrum of $\xi(\mathrm{t})$ "white noise." Krasnoff and Peskin (1971) assumed a short correlation time for $\xi(t)$. Gifford (1982) referred to an equivalent of $d W(t)$ as a purely random, uncorrelated function.

In his commentary on Gifford's paper, Sawford (1984) correctly specified the mathematical properties of $d W(t)$ and called it "... a Gaussian white-noise stochastic process." Wilson et al., (1981) identified a finite-difference equivalent of $d W(t)$ as a random number from a Gaussian distribution. Legg and Raupach (1982) cited Arnold (1974), called $\xi(t)$ "white noise," and gave its statistical properties, Wilson et al., (1983) did the same as Wilson et al., (1981). Thomson (1984) called $\xi(\mathrm{t})$ a random uncorrelated function and his equivalent of $d W(t)$ was used with non-Gaussian as well as Gaussian distributions. Sawford (1985) repeated what he wrote in 1984. van Dop et al. (1985) called $d W(t)$ a random increment, gave its mathematical properties, and mentioned that $d W(t) / \mathrm{dt}$ does not exist if accelerations are instantaneous. They cited Arnold (1974) and van Kampen (1981). Sawford (1986) cited van Kampen and Gardiner (1983) in treating $d W(t)$ as both a Gaussian and non-Gaussian process. Thomson (1987) identified $d W(t)$ as an increment of a Wiener process and cited Arnold and other works on stochastic processes.

The above review shows that it took time for workers in fluid dynamics and the atmospheric sciences to change from general word descriptions to statistical descriptions of $\xi(\mathrm{t})$ and $d W(t)$, and that it took even longer to cite mathematical works on stochastic processes. No one from Obukhov (1959) through Thomson (1987) explicitly describes the relation between white noise $\xi(t)$ and the Wiener process $d W(t)$ [see Eqs. (21a-b)]. Rather they change from the differential form of the Langevin equation as in Eq. (3) to its differential form as in Eq. (22).

\section{Drift Corrections for Inhomogeneous Turbulence}

The Langevin equation as in Eq. (22),

$$
d u=-\left(u / \tau_{L}\right) d t+\sigma_{u}\left(2 / \tau_{L}\right)^{\frac{1}{2}} d W(t)
$$

is based on the assumption that the turbulence is both stationary and homogeneous. Geophysical turbulence may be approximately stationary for periods of time, but (as in boundary layers) is 
generally not homogeneous. Workers have applied the above equation to realistic situations and discovered, on occasion, that the results were clearly nonphysical. As noted by Thomson (1984), this equation has been used with success when $\tau_{L}$-but not $\sigma_{u}$-varied with spatial coordinates (e.g., height). The bad results with variable $\sigma_{u}$ are best illustrated by means of an idealized test problem with a nonuniform velocity variance profile and an upwind boundary condition consisting of a uniform species concentration profile. The use of the above equation results in increased species concentrations in regions with low variance and decreased concentrations elsewhere, in violation of the second law of thermodynamics.

Wilson et al. (1981), hereafter WTK, wrote the equivalent of the Langevin equation in terms of dimensionless velocity ratios in which the reference conditions were those at a selected height. They used Lagrangian similarity theory to scale their results to other elevations. Subsequently Wilson et al. (1983), hereafter WLT, and Sawford (1985) rewrote the WTK equation in terms of the square root of the velocity variance:

$$
d\left(\frac{w}{\sigma_{w}}\right)=-\left(\frac{w}{\sigma_{w}}\right) \frac{d t}{\tau_{L}}+\left(\frac{2}{\tau_{L}}\right)^{\frac{1}{2}} d W(t)+\frac{d \sigma_{w}}{d z} d t
$$

where $w$ is the vertical velocity fluctuation, $\sigma_{w}^{2}$ is its variance, $z$ is the vertical coordinate, and the last term in the right is the drift correction proposed by WTK. This is Eq. (2) in the Introduction. From elementary calculus,

$$
d\left(\frac{w}{\sigma_{w}}\right)=\frac{d w}{\sigma_{w}}-w \frac{d \sigma_{w}}{\sigma_{w}^{2}}
$$

In addition,

$$
d z=w d t
$$

Then Eq. (27) can be rewritten as

$$
\frac{d w}{\sigma_{w}}-\left(\frac{w}{\sigma_{w}}\right)^{2} \frac{d \sigma_{w}}{d z} d t=-\left(\frac{w}{\sigma_{w}}\right) \frac{d t}{\tau_{L}}+\left(\frac{2}{\tau_{L}}\right)^{\frac{1}{2}} d W(t)+\frac{d \sigma_{w}}{d z} d t
$$

Multiplying by $\sigma_{w}$ and combining the terms with $d t$ gives

$$
d w=\left\{-\frac{w}{\tau_{L}}+\sigma_{w}\left[\left(\frac{w}{\sigma_{w}}\right)^{2}+1\right] \frac{d \sigma_{w}}{d z}\right\} d t+\sigma_{w}\left(\frac{2}{\tau}\right)^{\frac{1}{2}} d W(t)
$$

Therefore the WTK model includes not only the explicit drift correction $\left|\sigma_{w}\left(d \sigma_{w} / d z\right) d t\right|$ but an implicit correction $\left[\sigma_{w}\left(w / \sigma_{w}\right)^{2}\left(d \sigma_{w} / d z\right) d t\right]$.

Legg and Raupach (1982), hereafter LR, proposed a different drift correction which we will add to Eq. (26) with $u$ changed to $w$,

$$
d w=-\left(w / \tau_{L}\right) d t+\sigma_{w}\left(2 / \tau_{L}\right)^{\frac{1}{2}} d W(t)+\left(d \sigma_{w}{ }^{2} / d z\right) d t .
$$

If we combine the terms with $d t$ and use some elementary calculus on the quantity $\left(d \sigma_{w}^{2}\right)$,

$$
d w=\left(-\frac{w}{\tau_{L}}+2 \sigma_{w} \frac{d \sigma_{w}}{d z}\right) d t+\sigma_{w}\left(\frac{2}{\tau_{L}}\right)^{\frac{1}{2}} d W(t)
$$


It is clear from Eqs. (27), (30a-b), and (31a-b) that the LR drift correction is twice the explicit correction of WTK. It is also clear that the LR correction is equal to the sum of the explicit and implicit WTK corrections if $\left\langle w^{2}\right\rangle=\sigma_{w}^{2}$.

WLT demonstrated the analytical relation between the WTK and LR drift corrections, but in a more awkward manner than that above. They also showed that the two models give almost identical results under conditions of slightly inhomogeneous turbulence where $\left\langle w^{2}\right\rangle \approx \sigma_{w}^{2}$. Sawford (1985) showed the above analytic relation between the WTK and LR models without citing WLT, but he did so in his 1986 paper.

The most important contribution of WLT was their discovery that a simple modification of how WTK applied their model resulted in far better performance in strongly inhomogeneous turbulence than either the original WTK model, or that by LR. Wilson, Legg, and Thomson concluded their paper as follows:

"Though we can offer no proof that [the WTK model as modified by WLT] is 'correct' it seems worthy of investigation."

\section{Derivation of Drift Corrections}

Thomson of the WLT team continued the investigation of drift corrections and published a very important paper in 1984. Section 3 of that paper is related to the LR model and the WTK/WLT model is the subject of Section 5. We will summarize his Section 3 results, give a greatly expanded version of his Section 5, and use some of the Section 5 results to obtain the results of Section 3.

We start with the LR model, Eq. (31b), and symbolically let

$$
\mu=2 \sigma_{w} \frac{d \sigma_{w}}{d z} d t+\sigma_{w}\left(\frac{2}{\tau_{L}}\right)^{\frac{1}{2}} d W(t)
$$

so we get another version of the Langevin equation,

$$
d w=-\left(w / \tau_{L}\right) d t+\mu(t)
$$

where $\mu(t)$ is a new random function. In finite-difference form, Eq. (33a) is identical to the first part of Thomson's Eq. (2),

$$
w_{i+1}=\left[1-\Delta t / \tau_{L}(z)\right] w_{i}+\mu_{i+1},
$$

where $i$ indicates a time step. Thomson assumed that after a long time the marked particles of the released species will have the same steady-state probability density function in $(z, w)$ space as the air particles. He derived the moment-generating functions $\hat{g}(z, \theta)$ for the probability function $\mathrm{g}(z, w)$ for Eq. (33b) and then solved for the corresponding moment-generating function $\hat{\mathrm{f}}(\theta)$ for the probability density function $\mathrm{f}(\mu)$. In other words, he solved for the statistical properties of the forcing function $\mathrm{f}(\mu)$ as a function of the statistical properties of the velocity fluctuation $w$ defined by Eq. (33b). He found that, if the distribution of $w$ is Gaussian, the distribution of the forcing 
function $\mu$ is not-unless the turbulence is homogeneous $\left(d \sigma_{w} / d z=0\right)$. His solution for Gaussian turbulence is

$$
\begin{aligned}
\langle\mu\rangle & =\left(d \sigma_{w}^{2} / d z\right) \Delta t \\
\left\langle\mu^{2}\right\rangle & =\sigma_{w}^{2}\left(2 / \tau_{L}\right) \Delta t \\
\left\langle\mu^{3}\right\rangle & =3 \sigma_{w}^{2}\left(d \sigma_{w}^{2} / d z\right) \Delta t \\
\left\langle\mu^{n}\right\rangle & =0 \text { for } n>3 .
\end{aligned}
$$

The LR model, Eq. (31a-b), is based on the assumption that $\mu$ is Gaussian; hence Legg and Raupach used only the first two moments given in Eq. (34). The first moment in Eq. (34) is the LR drift correction and the square root of the second moment is the coefficient of $d W(t)$ in the LR model.

Thomson showed in a series of numerical simulations that the use of all three nonzero moments in Eq. (34) to define $\mu$ in Eq. (33b) improved the results over those of the two-moment LR model in moderately inhomogeneous turbulence. He also showed that the performance of the threemoment model of Eq. (34) was not good in severely inhomogeneous turbulence. He noted that the generation of prescribed non-Gaussian random variables is difficult; consequently it is not easy to apply Eqs. (33b) and (34) to inhomogeneous situations.

We note that Thomson used a numerical procedure that approximated the first three moments for $\mu$ in Eq. (34) -and also generated higher moments (with unknown or unspecified values) of $\mu$. We will find that this is very significant and that Thomson was apparently not fully aware of the implications at that time.

We now shift our attention to the WTK/WLT model, Eq. (30b), and symbolically let

$$
\mu=\sigma_{w}\left(d \sigma_{w} / d z\right) d t+\sigma_{w}\left(2 / \tau_{L}\right)^{\frac{1}{2}} d W(t)
$$

so we get

$$
d w=\left[-\frac{w}{\tau_{L}}+\left(\frac{w^{2}}{\sigma_{w}}\right) \frac{d \sigma_{w}}{d z}\right] d t+\mu(t)
$$

This version of Eq. (30b) has the implicit drift correction in view and the explicit WTK correction is now a component of $\mu(t)$. In finite-difference form we get

$$
w_{i+1}=\left(1-\frac{\Delta t}{\tau_{L}(z)}\right) w_{i}+\frac{\Delta t}{\sigma_{w}}\left(\frac{d \sigma_{w}}{d z}\right) w_{i}^{2}+\mu_{i+1}
$$

This is equivalent to Thomson's Eq. (16) and the first parts of his Eqs. (17)-(19). We will follow, and present in much greater detail, Thomson's procedure for developing moment-geiserating functions for Eq. (36b).

The moment-generating function used by Thomson is defined by the following integral transform (Lukacs, 1960, pp. 13-21; Parzen, 1960, pp. 215-217):

$$
\psi(t)=\int_{-\infty}^{\infty} e^{t x} p(x) d x
$$


where $\psi$ is the moment-generating function, $\mathrm{p}$ is the probability density of the continuous variable $x$, and $t$ is a continuous parameter. Another moment-generating function used later by Thomson (1987) is the characteristic function (Lukacs, loc. cit.; Parzen, pp. 394-399):

$$
\phi(t)=\int_{-\infty}^{\infty} e^{i t x} p(x) d x
$$

where $\phi$ is the characteristic function and $i=\sqrt{-1}$. The moment-generating function $\psi$ is connected to the characteristic function by the relation (Lukacs, pp. 18, 130-136)

$$
\psi(i t)=\phi(t)
$$

Let us use $\omega=2 \pi x$ and substitute $\mathrm{f}(t)$ and $F(\omega)$ for $\phi(t)$ and $p(x)$, respectively, in Eq. (37b) to get

$$
f(t)=\frac{1}{2 \pi} \int_{-\infty}^{\infty} F(\omega) e^{i \omega t} d \omega
$$

which is the inverse Fourier tranform where t is time, $\omega$ is angular frequency in radians per unit time, and $F(\omega)$ is the Fourier transform defined by the integral

$$
F(\omega)=\int_{-\infty}^{\infty} \mathrm{f}(t) e^{-i \omega t} d t
$$

The characteristic function, Eq. (37b), has more general application than the moment-generating function, Eq. (37a). This is indicated by the title and contents of the book by Lukacs and the second volume of Feller (1971) which has only one index citation for the moment-generating function and all of Ch XV on characteristic functions. However, we will follow Thomson (1984) and use the moment-generating function defined by Eq. (37a).

Thomson split the numerical procedure for Eq. (36b) into three steps. The first is given by his Eq. (17) for an advection step at constant velocity:

$$
\begin{aligned}
w_{i+1}^{\prime} & =w_{i} \\
z_{i+1}^{\prime} & =z_{i}+w_{i} \Delta t \\
t_{i+1}^{\prime} & =t_{i}+\Delta t .
\end{aligned}
$$

The second is the first of two velocity-incrementing steps, keeping location and time constant. that is defined by his Eq. (18):

$$
\begin{aligned}
w_{i+1}^{\prime \prime} & =\left(1-\frac{\Delta t}{\tau_{L}}\right) w_{i+1}^{\prime}+\frac{\Delta t}{\sigma_{w}} \frac{\partial \sigma_{w}}{\partial z}\left(w_{i+1}^{\prime}\right)^{2}, \\
z_{i+1}^{\prime \prime} & =z_{i+1}^{\prime}, \\
t_{i+1}^{\prime \prime} & =t_{i+1}^{\prime} .
\end{aligned}
$$


The first part of Eq. (39) consists of the terms with $w$ in Eq. (36b). The second velocityincrementing step accounts for the addition of the random velocity increment $\mu$, as in Eq. (36b), and is his Eq. (19):

$$
\begin{aligned}
w_{i+1} & =w_{i+1}^{\prime \prime}+\mu_{i+1} \\
z_{i+1} & =z_{i+1}^{\prime \prime} \\
t_{i+1} & =t_{i+1}^{\prime \prime}
\end{aligned}
$$

We use Thomson's nomenclature and apply Eq. (37a) to the turbulent velocity fluctuations in Eqs. (38)-(40):

$$
\hat{g}(z, \phi)=\int_{-\infty}^{\infty} e^{w \phi} g(z, w) d w
$$

where $g$ is the probability density function of the particles of air or other species at location $z$ with velocity $w, \hat{g}$ is the moment-generating function, and $\phi$ is a continuous parameter. The companion moment-generating function for the random function $\mu$ in Eq. (40) is

$$
\hat{f}(\theta)=\int_{-\infty}^{\infty} e^{\mu \theta} f(\mu) d \mu
$$

where $f$ is the probability density of $\mu$ and $\hat{f}$ is the moment-generating function. Our task is to rewrite Eqs. (38)-(40) in terms of Eqs. (41a-b).

Thomson's Eq. (17) and our Eq. (38) define an advection step over time $\Delta t$ and space $\Delta z$ and constant velocity so $\Delta z=\omega_{i} \Delta t$. Thomson's procedure for describing this step in terms of moment-generating functions is outlined in the material between his Eqs. (4)-(6). He lets

$$
g_{i+1}^{\prime}(z, w)=g_{i}(z-w \Delta t, w)
$$

where $z=z_{i+1}$. Then he uses a Taylor's series expansion

$$
g_{i+1}^{\prime}(z, w)=g_{i}(z, w)-w_{i} \Delta t, \partial g_{i} / \partial z+O\left(\Delta t^{2}\right)
$$

with $z=z_{i+1}$ as before. This may be an example of a "backward equation" because "... probabilities depending on the initial position set is by backward (retrospective) equations ..." (Feller I, 1968,

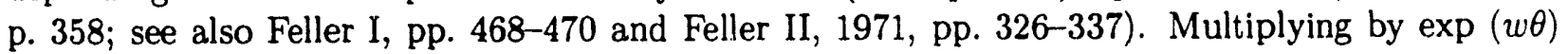
and integrating gives

$$
\int_{-\infty}^{\infty} e^{w \theta} g_{i+1}^{\prime} d w=\int_{-\infty}^{\infty} e^{w \theta} g_{i} d w-\Delta t \frac{\partial}{\partial z} \int_{-\infty}^{\infty} w e^{w \theta} g_{i} d w
$$

From elementary calculus,

$$
\partial e^{w \theta} / \partial \theta=w e^{w \theta}
$$

We apply Eqs. (41a) and (43) to Eq. (42c) to get Thomson's Eqs. (6) and (20):

$$
\hat{g}_{i+1}=\hat{g}_{i}-\Delta t\left(\partial^{2} \hat{g}_{i} / \partial \theta \partial z\right)+O\left(\Delta t^{2}\right) \text {. }
$$


Our next step is from Eq. (18) to Eq. (21) in Thomson's paper. He assumes that

$$
g_{i+1}^{\prime \prime}\left(z, w^{\prime \prime}\right) d w^{\prime \prime}=g_{i+1}^{\prime}\left(z, w^{\prime}\right) d w^{\prime}
$$

and applies this relation to his Eq. (18) [Our Eq. (39) ] as follows in Eq. (35a):

$$
\begin{aligned}
\hat{g}_{i+1}^{\prime \prime} & =\int_{-\infty}^{\infty} e^{w \prime \prime \theta} g_{i+1}^{\prime \prime}\left(z, w^{\prime \prime}\right) d w^{\prime \prime} \\
& =\int_{-\infty}^{\infty} e^{w \prime \theta} g_{i+1}^{\prime}\left(z, w^{\prime}\right) d w^{\prime} \\
& =\int_{-\infty}^{\infty} \exp \left\{\left[\left(1-\frac{\Delta t}{\tau_{L}}\right) w^{\prime}+\frac{\Delta t}{\sigma_{w}}\left(\frac{\partial \sigma_{w}}{\partial z}\right) w^{\prime 2}\right] \theta\right\} g_{i+1}^{\prime} d w^{\prime} \\
& =\int_{-\infty}^{\infty} \exp \left\{w^{\prime} \theta-\Delta t\left[\frac{w^{\prime}}{\tau_{L}}-\frac{1}{\sigma_{w}}\left(\frac{\partial \sigma_{w}}{\partial z}\right) w^{\prime 2}\right] \theta\right\} g_{i+1}^{\prime} d w^{\prime} \\
& =\int_{-\infty}^{\infty} e^{w^{\prime} \theta} \exp \left\{-\Delta t\left[\frac{w^{\prime}}{\tau_{L}}-\frac{1}{\sigma_{w}}\left(\frac{\partial \sigma_{w}}{\partial z}\right) w^{\prime 2}\right] \theta\right\} g_{i+1}^{\prime} d w^{\prime}
\end{aligned}
$$

We expand the second exponential in terms of $\Delta t$ and drop terms of order $\Delta t^{2}$ and higher:

$$
\begin{aligned}
\hat{g}_{i+1}^{\prime \prime}= & \int_{-\infty}^{\infty} e^{w / \theta}\left[1-\frac{\Delta t \theta w^{\prime}}{\tau_{L}}+\frac{\Delta t}{\sigma_{w}}\left(\frac{\partial \sigma_{w}}{\partial z}\right) \theta w^{\prime^{2}}\right] g_{i+1}^{\prime} d w^{\prime} \\
= & \int_{-\infty}^{\infty} e^{w \prime \theta} g_{i+1}^{\prime} d w^{\prime} \\
& -\frac{\Delta t \theta}{\tau_{L}} \int_{-\infty}^{\infty} w^{\prime} e^{w \prime \theta} g_{i+1}^{\prime} d w^{\prime} \\
& +\frac{\Delta t \theta}{\sigma_{w}}\left(\frac{\partial \sigma_{w}}{\partial z}\right) \int_{-\infty}^{\infty}{w^{\prime}}^{2} e^{w / \theta} g_{i+1}^{\prime} d w^{\prime} .
\end{aligned}
$$

We use Eq. (43) with the above to get

$$
\begin{aligned}
\hat{g}_{i+1}^{\prime \prime}= & \int_{-\infty}^{\infty} e^{w / \theta} g_{i+1}^{\prime} d w^{\prime} \\
& -\frac{\Delta t \theta}{\tau_{L}} \frac{\partial}{\partial \theta} \int_{-\infty}^{\infty} e^{w / \theta} g_{i+1}^{\prime} d w^{\prime} \\
& +\frac{\Delta t \theta}{\sigma_{w}}\left(\frac{\partial \sigma_{w}}{\partial z}\right) \frac{\partial^{2}}{\partial \theta^{2}} \int_{-\infty}^{\infty} e^{w / \theta} g_{i+1}^{\prime} d w^{\prime} .
\end{aligned}
$$


We apply Eq. (41a) to the above to get Thomson's Eq. (21):

$$
\hat{g}_{i+1}^{\prime \prime}=\hat{g}_{i+1}^{\prime}-\frac{\Delta t \theta}{\tau_{L}} \frac{\partial \hat{g}_{i+1}^{\prime}}{\partial \theta}+\frac{\Delta t \theta}{\sigma_{w}}\left(\frac{\partial \sigma_{w}}{\partial z}\right) \frac{\partial^{2} \hat{g} \prime_{i+1}}{\partial \theta^{2}}+O\left(\Delta t^{2}\right)
$$

The first part of Thomson's Eq. (19) and our Eq. (40) is an expression for the sum of two random variables, $w_{i+1}^{\prime \prime}$ and $\mu_{i+1}$. The moment-generating function for the sum of these variables is the product of their moment-generating functions (Lukacs, 1960, pp. 43-47; Parzen, 1960, p. 404; Gardiner, 1983, p. 33). Then from Eqs. (40) and (41a-b),

$$
\hat{g}_{i+1}(z, \theta)=\hat{g}_{i+1}^{\prime \prime}(z, \theta) \hat{f}(\theta)
$$

which is Thomson's Eq. (22). We combine Eqs. (44), (47), and (48) to get the following:

$$
\begin{aligned}
\hat{g}_{i+1} & =\left[\hat{g}_{i+1}^{\prime}-\frac{\Delta t \theta}{\tau_{L}} \frac{\partial \hat{g}_{i+1}^{\prime}}{\partial \theta}+\frac{\Delta t \theta}{\sigma_{w}}\left(\frac{\partial \sigma_{w}}{\partial z}\right) \frac{\partial^{2} \hat{g}_{i+1}^{\prime}}{\partial \theta^{2}}+O\left(\Delta t^{2}\right)\right] \hat{f}(\theta), \\
& =\left[\hat{g}_{i}-\Delta t \frac{\partial^{2} \hat{g}_{i}}{\partial \theta \partial z}-\frac{\Delta t}{\tau_{L}} \theta \frac{\partial \hat{g}_{i+1}^{\prime}}{\partial \theta}+\frac{\Delta t}{\sigma_{w}}\left(\frac{\partial \sigma_{w}}{\partial z}\right) \theta \frac{\partial^{2} \hat{g}_{i+1}^{\prime}}{\partial \theta^{2}}\right] \hat{f}(\theta)+O\left(\Delta t^{2}\right), \\
& =\left[\hat{g}_{i}-\frac{\Delta t}{\tau_{L}} \theta \frac{\partial \hat{g}_{i+1}^{\prime}}{\partial \theta}-\Delta t \frac{\partial^{2} \hat{g}_{i}}{\partial \theta \partial z}+\frac{\Delta t}{\sigma_{w}}\left(\frac{\partial \sigma_{w}}{\partial z}\right) \theta \frac{\partial^{2} \hat{g}_{i+1}^{\prime}}{\partial \theta^{2}}\right] \hat{f}(\theta)+O\left(\Delta t^{2}\right)
\end{aligned}
$$

which is Thomson's equation just above his Eq. (23). We follow him in setting $\hat{g}_{i+1}=\hat{g}_{i}=\hat{g}_{a}$ but we will drop his subscript "a" for convenience. We divide the above by $\hat{g}$ to get

$$
1=\left[1-\frac{\Delta t}{\tau_{L}} \frac{\theta}{\hat{g}} \frac{\partial \hat{g}}{\partial \theta}-\frac{\Delta t}{\hat{g}} \frac{\partial^{2} \hat{g}}{\partial \theta \partial z}+\frac{\Delta t}{\sigma_{w}}\left(\frac{\partial \sigma_{w}}{\partial z}\right) \frac{\theta}{\hat{g}} \frac{\partial^{2} \hat{g}}{\partial \theta^{2}}\right] \hat{f}(\theta)+O\left(\Delta t^{2}\right)
$$

We solve for $\hat{f}(\theta)$ and expand in terms of $\Delta t$ to get Thomson's Eq. (23):

$$
\hat{f}(\theta)=1+\frac{\Delta t}{\tau_{L}} \frac{\theta}{\hat{g}} \frac{\partial \hat{g}}{\partial \theta}+\frac{\Delta t}{\hat{g}} \frac{\partial^{2} \hat{g}}{\partial \theta \partial z}-\frac{\Delta t}{\sigma_{w}}\left(\frac{\partial \sigma_{w}}{\partial z}\right) \frac{\theta}{\hat{g}} \frac{\partial^{2} \hat{g}}{\partial \theta^{2}}+O\left(\Delta t^{2}\right)
$$

This is the moment-generating function for the random forcing function $\mu$ in terms of the momentgenerating function for this turbulent velocity fluctuation $w$.

Thomson assumed a Gaussian probability distribution $g(z, w)$ :

$$
g=\exp \left\{-\frac{1}{2}\left[\frac{w}{\sigma_{w}(z)}\right]^{2}\right\} / \sigma_{w}(z) \sqrt{2 \pi} .
$$

The corresponding moment-generating function $\hat{g}(z, \theta)$ is (Parzen, 1960, pp. 220-221)

$$
\hat{g}=\exp \left[\frac{1}{2} \theta^{2} \sigma_{w}^{2}(z)\right]
$$

Thomson assumed a variable density fluid so his equivalent of Eq. (53a) was

$$
\hat{g}=\rho(z) \exp \left[\frac{1}{2} \theta^{2} \sigma_{w}^{2}(z)\right]
$$


but for the sake of simplicity we will use Eq. (53a). The following are obtained by differentiating Eq. (53a):

$$
\begin{gathered}
\frac{\theta}{\hat{g}} \frac{\partial \hat{g}}{\partial \theta}=\theta^{2} \sigma_{w}^{2}=\frac{\sigma_{w}}{\hat{g}} \frac{\partial \hat{g}}{\partial \sigma_{w}}, \\
\frac{\theta}{\hat{g}} \frac{\partial^{2} \hat{g}}{\partial \theta^{2}}=\theta \sigma_{w}^{2}\left(1+\theta^{2} \sigma_{w}^{2}\right) \\
\frac{1}{\hat{g}} \frac{\partial^{2} \hat{g}}{\partial \theta \partial z}=\theta \sigma_{w} \frac{\partial \sigma_{w}}{\partial z}\left(2+\theta^{2} \sigma_{w}^{2}\right) .
\end{gathered}
$$

We substitute Eqs. (54a-c) into Eq. (51) to get the equivalent of the fourth equation after Thomson's Eq. (23):

$$
\begin{aligned}
\hat{f}(\theta)=1 & +\frac{\Delta t}{\tau_{L}} \theta^{2} \sigma_{w}^{2} \\
& +\Delta t \frac{\partial \sigma_{w}}{\partial z} \theta \sigma_{w}\left(2+\theta^{2} \sigma_{w}^{2}\right) \\
& -\frac{\Delta t}{\sigma_{w}} \frac{\partial \sigma_{w}}{\partial z} \theta \sigma_{w}\left(1+\theta^{2} \sigma_{w}^{2}\right), \\
=1 & +\frac{\Delta t}{\tau_{L}} \theta^{2} \sigma_{w}^{2}+\Delta t \frac{\partial \sigma_{w}}{\partial z} \theta \sigma_{w}\left(2+\theta^{2} \sigma w^{2}-1-\theta^{2} \sigma_{w}^{2}\right), \\
=1 & +\frac{\Delta t}{\tau_{L}} \theta^{2} \sigma_{w}^{2}+\Delta t \frac{\partial \sigma_{w}}{\partial z} \theta \sigma_{w}, \\
=1 & +\Delta t \theta \sigma_{w} \frac{\partial \sigma_{w}}{\partial z}+\frac{\Delta t}{\tau_{L}} \theta^{2} \sigma_{w}^{2}+O\left(\Delta t^{2}\right) .
\end{aligned}
$$

This is the equation for the moment-generating function for the random forcing function $\mu$ in Eqs. (36b) and (40).

The moments of $\mu$ are obtained by differentiating $\dot{f}(\theta)$ with respect to $\theta$ and then setting $\theta=\theta$ (Parzen, 1960, p. 217; Gardiner, 1983, p. 32). The first moment of $\mu$ from Eq. (55) is

$$
<\mu>=\left[\frac{\partial \hat{f}(\theta)}{\partial \theta}\right]_{0=0}=\Delta t \sigma_{w} \frac{\partial \sigma_{w}}{\partial z}+O\left(\Delta t^{2}\right) .
$$

The second moment is

$$
<\mu^{2}>=\left[\frac{\partial \hat{f}(\theta)}{\partial \theta}\right]_{\theta=0}=\frac{2 \Delta t \sigma_{w}^{2}}{\tau_{L}}+O\left(\Delta t^{2}\right) .
$$

The third moment of $\mu$ is zero so $f(\mu)$ is Gaussian.

Therefore we can let

$$
\mu=<\mu>+\left(<\mu^{2}>\right)^{1 / 2} \Delta W(t)
$$


where $\Delta W(t)$ is a scaled, dimensionless, Gaussian random process with variance equal to unity. Then from Eqs. (56a-b) and (57) in combination with Eq. (36b) we get

$$
\begin{aligned}
w_{i+1}=\left(1-\frac{\Delta t}{\tau_{L i}}\right) w_{i} & +\frac{\Delta t}{\sigma_{w i}} \frac{\partial \sigma_{w}}{\partial z} w_{i}^{2} \\
& +\Delta t \sigma_{w i} \frac{\partial \sigma_{w}}{\partial z}+\sigma_{w i}\left(\frac{2 \Delta t}{\tau_{L i}}\right)^{1 / 2} \Delta W(t)_{i+1} \\
= & \left(1-\frac{\Delta t}{\tau_{L i}}\right) w_{i}+\Delta t \sigma_{w}\left[\left(\frac{w_{i}}{\sigma_{w_{i}}}\right)^{2}+1\right] \frac{\partial \sigma_{w}}{\partial z}+\sigma_{w i}\left(\frac{2 \Delta t}{\tau_{L i}}\right)^{1 / 2} \Delta W(t)_{i+1}
\end{aligned}
$$

which is the finite-difference version of Eq. (30b), the WTK/WLT model! The explicit drift correction of this model is therefore equal to the mean value of the random function $\mu$ in Eq. (36b).

We have shown that the random forcing function in the WTK/WLT model [Eqs. (27), (30b), (36b), and (56a-b) $-(58)]$ is Gaussian if the turbulent velocity fluctuations are Gaussian. We have also noted that Thomson found that, if this turbulence is Gaussian, the random forcing function in the LR model [Eqs. (31a-b), (33b), and (34)] is not Gaussian, contrary to the original formulation by Legg and Raupach. We will now discover the reason for the non-Gaussian forcing function in the LR model as modified by Thomson.

In the preceding concerning the WTK/WLT model, the terms with $\partial \sigma_{\omega} / \partial z$ in Eq. (36b), (39), (47), and (51) are a consequence of the implicit drift correction in this model. These equations, with the $\partial \sigma_{\omega} / \partial z$ term removed, can be used for the analysis of the LR model. We will do this in deriving the moments given by Thomson in his Table 1 and our Eq. (34). We start with the appropriate form of Eq. (51):

$$
\hat{f}(\theta)=1+\frac{\Delta t}{\tau_{l}} \frac{\theta}{\hat{g}} \frac{\partial \hat{g}}{\partial \theta}+\frac{\Delta t}{\hat{g}} \frac{\partial^{2} \hat{g}}{\partial \theta \partial z}+O\left(\Delta t^{2}\right),
$$

which is Thomson's Eq. (19). We assume that Eqs. (52) and (53a) for Gaussian turbulence apply and use Eqs. (54a) and (54c), but not Eq. (54b), to get the following replacement for Eq. (55):

$$
\begin{aligned}
\hat{f}(\theta)= & 1+\frac{\Delta t}{\tau_{L}} \theta^{2} \sigma_{w}^{2}+\Delta t \frac{\partial \sigma_{w}}{\partial z} \theta \sigma_{w}\left(2+\theta^{2} \sigma_{w}^{2}\right), \\
= & 1+2 \Delta t \theta \sigma_{w} \frac{\partial \sigma_{w}}{\partial z}+\frac{\Delta t}{\tau_{L}} \theta^{2} \sigma_{w}^{2}+\Delta t \theta^{3} \sigma_{w}^{3} \frac{\partial \sigma_{w}}{\partial z}, \\
= & 1+\Delta t \theta \frac{\partial \sigma_{w}^{2}}{\partial z}+\frac{\Delta t}{\tau_{L}} \theta^{2} \sigma_{w}^{2}+\Delta t \theta^{3} \frac{\sigma_{w}^{2}}{2} \frac{\partial \sigma_{w}^{2}}{\partial z}+O\left(\Delta t^{2}\right) .
\end{aligned}
$$

The moments of $\mu$ from Eq. (60) are

$$
\begin{aligned}
& <\mu>=\left[\frac{\partial \hat{f}(\theta)}{\partial \theta}\right]_{\theta=0}=\Delta t \frac{\partial \sigma_{w}^{2}}{\partial z}+O\left(\Delta t^{2}\right), \\
& <\mu^{2}>=\left[\frac{\partial^{2} \hat{f}(\theta)}{\partial \theta^{2}}\right]_{\theta=0}=\frac{2 \Delta t}{\tau_{L}} \sigma_{w}^{2}+O\left(\Delta t^{2}\right), \\
& <\mu^{3}>=\left[\frac{\partial^{3} \hat{f}(\theta)}{\partial \theta^{3}}\right]_{\theta=0}=3 \Delta t \sigma_{w}^{2} \frac{\partial^{2} \sigma_{w}^{2}}{\partial z}+O\left(\Delta t^{2}\right), \\
& <\mu^{n}>=\left[\frac{\partial^{n} \hat{f}(\theta)}{\partial \theta^{n}}\right]_{\theta=0}=0+O\left(\Delta t^{2}\right) \text { for } n>3 .
\end{aligned}
$$


These are Thomson's results for the LR model which we gave above in Eq. (34).

What are the origins of the moments of $\mu$ for the WTK/WLT model [Eqs. (56a-b)] and the LR model [Eqs. $(61 \mathrm{a}-\mathrm{d})$ ]? The immediate sources are the terms with $\theta$ and $\theta^{2}$ in Eq. (55) and the terms with $\theta, \theta^{2}$, and $\theta^{3}$ in Eq. (60), respectively. Equations (54a-c) are important links backward from Eqs. (55) and (60) to Eqs. (36b) and (33b), respectively.

The second moment is identical for the two models. It has its origin in the identical $\theta^{2}$ terms in Eqs. (55) and (60) through Eq. (54a) and other equations to the $w_{i} \Delta t / \tau_{L}$ terms in Eqs. (36b) and (33b), respectively. With reference to the discussion between Eqs. (13) and (14), the second moment of the random forcing function is related to the equilibrium between correlation and decorrelation of the Lagrangian particle velocity.

The first and third moments are different for the models. Both moments involve the quantity $\partial \sigma_{u} / \partial z$ (or $\partial \sigma_{w}^{2} / \partial z$ ) which is a measure of the inhomogeneity of the turbulence. The differences between the WTK/WLT sequence Eqs. (51), $(54 \mathrm{a}-\mathrm{c})$, and (55) and the LR sequence Eq. (59), (54a), (54c), and (60) are obvious. Note in the preliminary version of the final form of Eq. (55) that, compared to the preliminary to Eq. (60), the coefficient of the $\theta$-term for the first moment is cut in half and that of the $\theta^{3}$-term for the third moment is reduced to zero. This is a consequence of the presence of the implicit drift correction in the WTK/WLT model. This implicit correction is related to the normalization of the Lagrangian velocity by the square root of its variance in the original formulation of the model (see Eqs. (27)-(30b)).

The above is a sketch of how the moment-generating functions relate the random forcing function to the turbulent velocity field-and visa versa. The major contribution of Thomson (1984) is the procedure to get outputs from the Langevin equation that are statistically consistent with its inputs. We will see that he did not realize at the time that his solution for a Gaussian random forcing function for the WTK/WLT model [Eqs. (56a)-(58)] is mathematically legitimate whereas his solution for a non-Gaussian forcing function for the LR model [Eqs. (33b), (34), and $(61 \mathrm{a}-\mathrm{d})]$ is not.

\section{Gaussian and Non-Gaussian Distributions}

We begin with the respective comments of van Dop et al. (1985) and Sawford (1986): "It is, however, generally observed that in inhomogeneous turbulent flows, the turbulence is not Gaussian," and "...for many flows of practical interest (for example the atmospheric convective boundary layer) the turbulence is strongly inhomogeneous and is non-Gaussian." In the preceding section we studied the work of Thomson (1984). He assumed that two different Langevin models with drift corrections (the LR and WTK/WLT models) generated Gaussian turbulence and discovered that the LR model required a non-Gaussian forcing function while the WTK/WLT model required Gaussian forcing. We will now review the work of earlier and later investigations with respect to Gaussian and non-Gaussian distributions.

WTK (Wilson, Thurtell, and Kidd, 1981) used a random noise generator for the forcing function in their numerical simulations; its output was approximately Gaussian. LR (Legg and Raupach. 1982 ) defined their forcing function, $\xi(t)$, as Gaussian white noise and used a Gaussian forcing 
function in their numerical simulations. WLT (Wilson, Legg, and Thomson, 1983) did essentially the same in their comparison of the WTK and LR models and their proposal of the WTK/WLT model. Apparently none of these workers considered the statistical properties of the turbulence generated by their models.

The available evidence indicates that Thomson (1984) was the first to consider the probability density functions of both the input (the forcing function) and the output (the turbulent velocity distribution) of Langevin diffusion models. He assumed outputs of Gaussian turbulence, as noted above, and found that the LR model required non-Gaussian forcing while the WTK/WLT model was self-consistent with Gaussian forcing. As we have seen in Eqs. (32)-(34) and (59)-(61), his non-Gaussian solution for the LR model had, for first-order terms in $\Delta t$, non-zero values for the first three moments and zero values for all the higher moments. For his numerical simulations, he used a combination of two Gaussian distributions to approximate the first three momerns and let the higher moments have whatever (not specified) values resulted. This approximation helped in slightly-to-moderately inhomogenous turbulence but failed in strongly inhomogeneous turbulence where the WTK/WLT model did very well. He concluded Section 3 of his paper as follows: "To obtain good results it is necessary to ensure that more than just the first three moments of $\mu$ are correct. Such a random variable is difficult to generate, and so Eq. (2) la combination of our Eqs. (38)-(40) without the $\partial \sigma_{w} / \partial z$ term] cannot easily be applied to situations as inhomogeneous as this." He later discovered that "such a random variable" is not difficult but impossible to generate.

van Dop et al. (1985) and Sawford (1986) cited Thomson (1984) and noted that random, nonGaussian forcing functions like the one Thomson developed for the LR model are not realizable and can only be approximated. This is recognized by Thomson in his 1987 paper in which he required Gaussian forcing in his evaluation of criteria for a "good" Langevin model. He also demonstrated that a self-consistent Langevin model can generate non-Gaussian turbulence from Gaussian forcing.

\section{Realizable Probability Distributions}

It is in order for us to go into the problem of the realizability of probability distributions with more breadth and depth than van Dop et al. (1985), Sawford (1986), and Thomson (1987). These authors used the Fokker-Planck equation, the Eulerian equivalent of the Langevin equation, in much of their analyses. To be specific they used the bivariate Fokker-Planck equation which is also called the Kramers equation or the Klein-Kramers equation because Klein derived it in 1922 and Kramers applied it in 1940 (van Kampen, 1981, p. 233; Gardiner, 1983, pp. 155-156).

We begin with the LR version of the Langevin equation, Eq. (31b), which we rewrite as

$$
d w=\left(-\frac{w}{\tau_{L}}+a_{1}\right) d t+a_{2}^{1 / 2} d W(t)
$$

where

$$
\begin{aligned}
& a_{1}=d \sigma_{w}^{2} / d z, \\
& a_{2}=2 \sigma_{w}^{2} / \tau_{L} .
\end{aligned}
$$


We note that $a_{1} d i$ and $a_{2} d t$ are respectively equal and identical to the first two of the three moments clerived by Thomson (1984) that are given here in Eqs. (34) and $(61 \mathrm{a}-\mathrm{c})$. The corresponding FokkerPlanck equation is (van Kampen, pp. 237-236; Gardiner, pp. 96-98; van Dop et al., Eqs. (18) and $(22))$.

$$
\frac{\partial P}{\partial t}+\frac{\partial}{\partial z}(w P)=-\frac{\partial}{\partial w}\left[\left(a_{1}-\frac{w}{\tau_{L}}\right) P\right]+\frac{1}{2} \frac{\partial^{2}}{\partial w^{2}}\left(a_{2} P\right)
$$

Here $P=P(z, w, t)$, the joint probability that a particle is observed in the internal $(z, z+d z)$ with a velocity in the range $(w, w+d w)$. According to van Kampen (p. 210), the stationary solution of Eq. (63) is Gaussian because of the term $\left(-w / \tau_{L}\right)$.

In Section 5 on skewed random acceleration van Dop et al. noted Thomson's (1984) solution for the third moment [Eq. (61c)] of the random forcing function. They closed this section with these remarks: "Apart from the numerical difficulties which appear when a skewed random forcing is applied, some other problems occur. It is straight forward to carry out the analysis leading to the Fokker-Planck equation for a random acceleration with arbitrary moments (the Kramers-Moyal expansion) ... However, differential equations of higher than second order cannot any longer describe the evolution of a probability density function [van Kampen, $1981 ; \ldots$ ) since the requirement that the probability distribution $P(z, w, t)$ is positive everywhere is violated." van Kampen (pp. 215 and 280) made similar comments, including this footnote on p. 280: "that no differential equation of higher order than Fokker-Planck can describe the evolution of a probability density rigorously has been proven by R.F. Pawula, Phys. Rev. 162, 186 (1967)."

The Kramers-Moyal expansion (van Kampen, p. 215; Gardiner, pp. 246-247 and 249-250) is a generalization of the Fokker-Planck equation with additional terms $\partial^{3}\left(a_{3} P\right) / \partial w^{3}, \partial^{4}\left(a_{4} P\right) / \partial w^{4}$, etc. Gardiner (p. 250) stated that there are limits to the validity of this expansion, and illustrated this with a case in which terms higher than the second vanish. Getting back to Pawula, he used the Schwarz inequality to prove that if it is assumed that $a_{n}=0$ for some even $n \geq 4$, it logically follows that $a_{n}=0$ for $n \geq 3$. As shown in Eqs. (61a-d), Thomson's (1984) solution for the forcing function in the LR model [Eqs. (31b)-(33b)] gave the result that $a_{n}=0$ for $n \geq 4$. Therefore this solution is not realizable. Thomson was, as we have noted, aware at that time of numerical difficulties in generating non-Gaussian forcing functions, but he made no mention of the problem of realizability. He did so, however, in his 1987 paper. He remarked in Section 2.3 that a Langevin model which requires non-Gaussian forcing "... must either be non-existent (in the sense that no random forcing exists with the required moments) or have discontinuous phase-space trajectories." He continued with the observation that the former applies to his 1984 solution for the LR model because it "... requires the first three moments of the random increments to be $O(\Delta t)$ with higher moments $O\left(\Delta t^{2}\right)$. Now any random variable $X$ must satisfy $\left(\overline{X^{3}}\right)^{2} \leq \overline{X^{2} X^{4}}$ Feller, 1971, pp. 152153 and 155 ]. Hence there is no random forcing with the required moments." (Thomson actually cited p. 151 in the 1966 edition of Feller.) This material in Feller concerns the Schwarz inequality, which is what Pawula used in his proof.

Sawford (1986) demonstrated that the results of van Dop et al. and Thomson (1984) are equivalent. He also discussed the possible use of the Kramers-Moyal expansion to higher order than the second (than the Fokker-Planck equation): "Truncation at any higher order is not self-consistent and results in nonphysical behaviour such as negative $P$. ... the theorum of Marcinkiewicz shows that either all but the first two cumulants of $[d W(t)]$ vanish or else there are an infinite number of nonvanishing cumulants [Gardiner, p. 36]. This automatically ensures that [the Kramers-Moyal 
expansion] is either truncated at $\left[\partial^{2} P / \partial w^{2}\right]$ or not at all and therefore that $F$-orrectly represents a probability distribution, even though in practice [truncation to only a few moments] it may only approximate the correct distribution." With respect to "in practice," Pawula and van Kampen agreed that higher-than-second order truncations are not necessarily useless, but they should be used with caution.

Robinson (1965) independently proved the theorem of Marcinkiewicz-and then was informed that he had duplicated some work published in 1938! We begin our study of this theorem by rewriting Eq. (37b) for this characteristic function (van Kampen, p. 6; Gardiner, p. 32):

$$
\hat{f}(\theta)=\int_{\infty}^{\infty} e^{i \theta w} p(w) d w=<\exp (i \theta w)>=\exp \left\{\sum_{m=1}^{\infty} \frac{(i \theta)^{m}}{m !} \mu_{m}\right\}=\sum_{m=0}^{\infty} \frac{(i \theta)^{m}}{m !} \mu_{m} .
$$

Here $w, \theta$, and $\mu$ have the same meanings as above in our expanded treatment of Thomson's (1984) solutions for the WTK/WLT and LR models. The last term on the right in Eq. (64) is the Taylor's series expansion of the preceding term. For $0 \leq m \leq 2$, Eq. (64) is equivalent to Eq. (55) for the WRK/WLT model and, for $0 \leq m \leq 3$, it is equivalent to Eq. (60) for the LR model. Robinson stated the Marcinkiewicz theorum as follows: "If $\exp [P(x)]$, where $P(x)$ is a polynomial, is a characteristic function then it follows that $P(x)=i a x-b x^{2}$ where $a$ and $b$ are real and $b>0$." Here $\exp [P(x)]$ corresponds to the next-to-the-last term in Eq. (64). We see here that Thomson's solution for the WTK/WLT model is in accord with the theorum and that his solution for the LR model violates the theorum.

In closing, Hänggi and Talkner (1980) emphasize that Marcinkiewicz's theorum implies the theorum of Pawula.

\section{Definitions of $\sigma_{w}$ and $\tau_{L}$ for Inhomogeneous Turbulence}

In view of the above, we have only one realizable, self-consistent Langevin model for use in stationary but inhomogeneous turbulence, the WTK/WLT model which may be written in the differential form of Eq. (30b),

$$
d w=\left\{-\frac{w}{\tau_{L}}+\sigma_{w}\left[\left(\frac{w}{\sigma_{w}}\right)^{2}+1\right] \frac{d \sigma_{w}}{d z}\right\} d t+\sigma_{w}\left(\frac{2}{\tau_{L}}\right)^{1 / 2} d W(t)
$$

or the finite-difference form of Eq. (58),

$$
w_{i+1}=\left(1-\frac{\Delta t}{\tau_{L i}}\right) w_{i}+\Delta t \sigma_{w i}\left[\left(\frac{w_{i}}{\sigma_{w i}}\right)^{2}+1\right] \frac{d \sigma_{w_{i}}}{d z}+\sigma_{w i}\left(\frac{2 \Delta t}{\tau_{L i}}\right)^{1 / 2} \Delta W(t)_{i+1}
$$

We need to reexamine the definitions of $\sigma_{w}$ and $\tau_{L}$ for this model because they were originally defined in the context of Eqs. (3) and (12)-(19) for stationary, homogeneous turbulence. Specifically, $\sigma_{w}^{2}$ (as $\sigma_{u}^{2}$ ) was defined as the variance of the Lagrangian velocity and $\tau_{L}$ was defined as the integral time scale of the Lagrangian velocity.

WTK (Wilson, Thurtell, and Kidd, 1981) called $\sigma_{w}$ the Eulerian turbulent velocity scale or rootmean-square vertical velocity which was height-dependent. They also stated that it was expected that satisfactory particle trajectories would be obtained with $\sigma_{L}(z(x))=\sigma_{w}(z)$, but $\sigma_{L}$ (apparently 
the Lagrangian velocity scale) does not appear explicitly in any of their equations. LR (Legg and Raupach, 1982) stated that $\sigma_{w}^{2}$ is the Lagrangian velocity variance, their drift correction term is $\partial\left\langle w_{E}^{2}\right\rangle / \partial z$ where $\left\langle w_{E}^{2}\right\rangle$ is the Eulerian velocity variance, and they assumed that $\sigma_{w}^{2}=\left\langle w_{E}^{2}\right\rangle$. WLT (Wilson, Legg, and Thomson, 1983) stated that $\sigma_{w}^{2}$ is the Eulerian velocity variance and that the Lagrangian length scale is $l(z)=\sigma_{w}(z) \tau_{L}(z)$ where $\tau_{L}$ is the Lagrangian time scale. (We will consider the various definitions of $\tau_{L}$ in a later paragraph.) Thomson (1984) defined $\sigma_{w}^{2}$ as the Eulerian velocity variance. WTK, LR, WLT, and Thomson were principals in the work that led to Eqs. (65a-b). Except for some ambiguity in LR, there is agreement among the above that $\sigma_{w}^{2}$ is the Eulerian velocity variance.

The survey paper of Sawford (1985) is confusing with respect to the definition of $\sigma_{w}$. In the Appendix (Nomenclature) $\sigma_{w}$ is defined as the Langevin [sic] velocity scale and $\sigma_{w E}^{2}$ is called the Eulerian vertical velocity variance. He cited Lumley (1962) for the equality $\sigma_{w}^{2}=\sigma_{w E}^{2}$ in stationary, homogeneous turbulence; Lumley showed that the Langrangian and Eulerian turbulence statistics are identical under these conditions. Sawford went on to state that $\sigma_{w}^{2}$ does not correspond to the Lagrangian velocity variance in inhomogeneous turbulence and that most authors take $\sigma_{w}^{2}=\sigma_{w E}^{2}$. He later stated that in strongly inhomogeneous turbulence $\left\langle w^{2}\right\rangle \neq \sigma_{w}^{2}$ (see the $\left(w / \sigma_{w}\right)^{2}$ terms in Eqs. (65a-b)). Perhaps what Sawford meant in the Appendix by $\sigma_{w}$ being the "Langevin velocity scale" is the meaning of $\sigma_{w}$ in the Langevin equation. If so, $\sigma_{w}^{2}$ is the Eulerian velocity variance which, under special conditions, is equal to the Lagrangian velocity variance.

van Dop et al. (1985) noted that, in inhomogeneous turbulence, the Lagrangian velocity variance $\left\langle w^{2}\right\rangle$ is equal to the fluid velocity variance $\left.\sigma_{w}^{2}\left(\equiv<u_{3}^{2}\right\rangle\right)$ where $u_{3}$ is the Eulerian vertical velocity fluctuation. They used $u$ for the fluid (Eulerian) velocity and $w$ for the particle (Lagrangian) velocity throughout their paper. Sawford (1986) used the same nomenclature for the fluid and particle velocities and gave the relation $\sigma_{w}=\left(\left\langle u_{3}^{2}\right\rangle\right)^{1 / 2}$ in connection with the WLT paper and Thomson (1984).

Novikov (1986) discussed the WTK/WLT model and defined $\sigma_{w}$ as the (density) weighted diffusion [sic] of Eulerian velocity. Elsewhere in connection with a more general form of the Langevin equation, he defined the fluctuation velocity of a particle as

$$
u^{\prime}=u-U
$$

where $u$ is the particle velocity and $U$ is the mean Eulerian velocity. This is consistent with Thomson (1987) who wrote an equation in tensor notation for the probability density function $g(\underline{x}, \underline{u}, t)$ for fluid particles in three-dimensional Gaussian turbulence. For one-dimensional, constant-density turbulence this relation can be written as

$$
g=\frac{1}{\sqrt{2 \pi}\left(\left\langle\left(u_{E}-U\right)^{2}\right\rangle\right)^{\frac{1}{2}}} \exp \left[-\frac{1}{2}\left(\frac{(u-U)^{2}}{\left\langle\left(u_{E}-U\right)^{2}\right\rangle}\right)\right]
$$

where $u$ is the Lagrangian velocity, $u_{E}$ is the Eulerian velocity, and $U$ is the mean Eulerian velocity. In summary, both Novikov and Thomson used the mean Eulerian velocity as the mean for both Lagrangian and Eulerian velocity statistics and $\sigma_{w}^{2}$ in Eqs. $(65 a-b)$ as the variance of the Eulerian velocity.

Now we consider definitions of $\tau_{L}$. WTK (1981) gave a mathematical definition for the Eulerian velocity time scale that is analogous to our Eqs. (12), (14), and (15) for the Lagrangian velocity 
integral time scale. They called $\tau_{L}$ the Lagrangian time scale and assumed it to be closely related to the Eulerian time scale. LR (1982) used relations equivalent to Eqs. (14) and (15) to define $\tau_{L}$ as the Lagrangian integral time scale. WLT (1983) referred back to WTK for the definition of $\tau_{L}$. Thomson (1984) called $\tau_{L}$ a time scale for the particle motion and noted that it is equal to the Lagrangian integral time scale in homogeneous turbulence. Sawford (1985) made the same statement about $\tau_{L}$ in homogeneous turbulence but stated that $\tau_{L}$ is better considered to be the local decorrelation time scale in inhomogeneous turbulence. van Dop et al. called $\tau_{L}$ the time scale over which the particle velocity is correlated; Sawford (1986) did the same. Novikov (1986) called $\tau_{L}$ a relaxation time. Thomson (1987) wrote, "In conditions of stronger inhomogeneity or unsteadiness $\left[\tau_{L}\right]$ is not the integral time scale but simply a (rather loosely defined) 'local decorrelation time scale'."

In summary, $\sigma_{w}^{2}$ in Eqs. $(65 \mathrm{a}-\mathrm{b})$ has an exact definition: it is the Eulerian velocity variance. On the other hand, $\tau_{L}$ in those equations is "loosely defined" as a "local decorrelation timescale." We also note that Novikov (1986) and Thomson (1987) used the mean Eulerian velocity to define the Lagrangian velocity fluctuations (see Eq. (67)).

\section{Interim Review and References}

This is a good point at which to pause and review what we have studied and learned. We need to know where we started, the route we took, and where we are now before we decide on our next course. It is premature to say where our journey will end. As part of our review, we will list the references we cited and call attention to those that are especially significant.

\section{Review}

The Langevin equation was the first stochastic differential equation and is widely used as an example (e.g., throughout Arnold (1974)). It includes a random forcing function (its input) whose statistical properties must be defined in order to obtain its statistical solution (its output). The earlier mathematical model of this function consisted of a series of random impulses with very short correlation times (e.g., Uhlenbeck and Ornstein, 1930). The current mathematical models are Gaussian "white noise" with its covariance defined as a Dirac delta function and its integral, the Wiener process, which is more generally used (Arnold, Ch3). The former is more realistic physically and the latter is mathematically convenient. The Wiener process, as a continuous function, is superior to discontinuous white noise for use in integrating stochastic differential equations. The Langevin equation was in use for more than 40 years before Ito formulated his calculus for integrating stochastic differential equations (Arnold, Ch4).

We use the Langevin equation to model the diffusion of "marked" particles in turbulent fluid flow. For stationary, homogeneous tubulence, the finite-difference form of Eq. (26) is

$$
u_{i+1}=\left(1-\Delta t / \tau_{L}\right) u_{i}+\sigma_{u i}\left(2 \Delta t / \tau_{L}\right)^{1 / 2} \Delta W_{N}(t)_{i+1}
$$

where $u$ is the Lagrangian particle velocity, $\tau_{L}$ is the integral time scale of $u, \sigma_{u}$ is the square root of the Eulerian (and, under these condition, the Lagrangian) velocity variance, $\Delta W_{N}(t)$ is the normalized incremental Wiener process with a variance of unity (instead of a variance of $d t$ 
as in Eq. (26)), and $i$ is the time step index. The first term on the right represents the "fading memory" or decorrelation of the velocity $u$ with the step from $i$ to $i+1$. We have not addressed the criterion for the relative values of $\Delta t$ and $\tau_{L}$; Sawford (1985) states that, typically, $\Delta t<\tau_{L} / 10$ for proper resolution of the memory effects. The last term on the right is the random velocity increment generated by flunctuating pressure forces with short correlation times on the order of the Kolmogorov time scale $\tau_{k}$. Because $\tau_{k}$ is extremely small,

$$
\tau_{L} / 10>\Delta t \gg \tau_{k}
$$

for practical numerical calculations. However, the numerical solutions with $\Delta t=\tau_{k}$ would be mathematically valid, but not physically valid until the integration had proceeded until $t \gg \tau_{k}$.

Turbulence can be approximately stationary, but it is generally not homogeneous. It was soon discovered that the use of Eq. (68) for inhomogeneous turbulence often led to clearly nonphysical results. Specifically, "marked" particles tended to accumulate in regions of low velocity variance. Drift correction terms, based on physical reasoning, were added on an ad hoc basis to the Langevin equation. We studied the WTK correction by Wilson, Thurtell, and Kidd (1981) and the LR version by Legg and Raupach (1982). A modification of the former, the WTK/WLT model, was found by Wilson, Legg, and Thomson (1983) to give the best performance of all in strongly inhomogeneous turbulence, but they could not explain why.

Thomson (1984) began the process of explanation. He used moment-generating functions (which are related to characteristic functions, a Fourier transform) to derive the moments of the random forcing functions for the LR and WTK/WLT versions of the Langevin equation. He found that, assuming Gaussian turbulence as the equation output, the required forcing function for the WTK/WLT model is Gaussian but that for the LR model is not. Specifically, he obtained nonzero values for the first two moments of the WTK/WLT forcing and the first three moments of the LR forcing; he found the values of all the higher moments to be zero for both models. (These values were for terms on the order of $\Delta t$ (see Eq. (68)); terms on the order of $\Delta t^{2}$ and higher were neglected.) In his numerical simulations to compare the two models, he was able to generate the required moments for the Gaussian WTK/WLT model but not for the non-Gaussian LR model. Specifically, he could get good approximations to the first three (nonzero) moments but not the higher (zero) moments of the LR model. He found that the performance of the WTK/WLT model was far superior in simply inhomogeneous turbulence, and he attributed this to the practical difficulties of generating the required non-Gaussian forcing for the LR model.

van Dop et al. (1985) and Sawford (1986) discussed Thomson's (1984) work and commented that approximating non-Gaussian distributions with a truncated series of moments can cause mathematical difficulties such as negative probability distributions. They cited van Kampen (1981) and Gardiner (1983), respectively. These references in turn and respectively, lead to the theorums of Pawula (1967) and Marcinkiewicz (Robinson, 1965; a more accessible paper than that published in 1938 by Marcinkiewicz). The common meaning of these theorems for us is that the probability density function defined by the first three moments of the LR forcing function (see Eqs. (61a-d)) that Thomson (1984) obtained does not exist. A realizeable probability density function must be defined by either an infinite number of moments (non-Gaussian) or a series truncated at the second moment (Gaussian). Thomson (1987) recognized the non-realizability of his 1984 solution and cited the 1966 edition of Feller (1971) as proof. (He used Gaussian forcing exclusively in the new work presented in his 1987 paper.) van Kampen (p. 280) comments that the use of moments higher than 
second-order is not incorrect or useless but that one should be cautious about drawing conclusions from the results. This is demonstrated by Thomson's (1984) numerical simulations in which the original LR model with two-moment random forcing did well in slightly inhomogeneous turbulence and the approximated three-moment version of the LR model did better than the two-moment version in moderately inhomogeneous turbulence. However, the approximated three-moment LR model did poorly in strongly inhomogeneous turbulence where the WTK/WLT model did well.

We therefore have one proven, self-consistent Langevin model for use in Gaussian inhomogeneous turbulence, Eq. (65b):

$$
w_{i+1}=\left(1-\frac{\Delta t}{\tau_{L i}}\right) w_{i}+\Delta t \sigma_{w i}\left[\left(\frac{w_{i}}{\sigma_{w i}}\right)^{2}+1\right] \frac{d \sigma_{w i}}{d z}+\sigma_{w_{i}}\left(\frac{2 \Delta t}{\tau_{L i}}\right)^{1 / 2} \Delta W(t)_{i+1}
$$

where $w$ is the Lagrangian velocity fluctuation referenced to the mean Eulerian velocity, $\sigma_{w}^{2}$ is the Eulerian velocity variance, $\tau_{L}$ is a local decorrelation time, $\Delta t$ is within the limits of Eq. (69), and $z$ is the vertical coordinate. The first moment of the Gaussian random forcing function is

$$
\Delta t \sigma_{w} d \sigma_{w} / d z
$$

and the second moment is

$$
2 \Delta t \sigma_{w}^{2} / \tau_{L}
$$

(see Eqs. $(56 a-b))$. The second moment, in the form of its square root, is the companion to the incremental Wiener process. The first moment is identical to the explicit drift correction of Wilson, Thurtell, and Kidd (1981), and it is associated with the " +1 " in the second term on the right in Eq. (70). The other part of this second term is associated with " $\left(w / \sigma_{w}\right)^{2}$ " and is the implicit drift correction which is a consequence of the normalization of $w$ by $\sigma_{w}$ in the original formulation of the WTK model (see Eqs. (27)-(30b)).

Some of the 32 references cited are especially significant or are worthy of study. The significance of Obukhov (1959) is that he "triggered" the development of the Langevin equation as a model for turbulent diffusion. Lin and Reid (1963) is an excellent summary of the statistical turbulence theory of that time, and it includes a pioneering effort to apply the Langevin equation to turbulent diffusion. Section 2 of Legg and Raupach (1982) is a clear exposition on the solution of the Langevin for stationary, homogeneous turbulence. Uhlenbeck and Ornstein (1930) is a good followon to Legg and Raupach, including their version of "white noise" as a series of random pulses with very short correlation times. Chapters 1 and 2 of the classic Chandrasekhar (1943) could be studied next with considerable benefit, but it would not be easy. For the modern treatment of "white noise" and the related "Wiener process," one should use Chapter VIII of van Kampen (1981), Chapters 1 and 4 of Gardiner (1983), and the Introduction and Chapter 3 of Arnold (1974) in that order. Wilson, Legg and Thomson (1983) is very important because of their "invention" of the WTK/WLT Langevin model for inhomogeneous turbulence, but it is poorly written. Thomson (1984) is perhaps the most important because he pioneered the use of moment-generating functions to obtain consistent inputs and outputs of the Langevin model (for inhomogeneous turbulence) as a stochastic differential equation. His Section 8 on the WTK/WLT model has stood the test of time even through his Section 3 relating to the LR model has not. The study of van Dop, Niewstadt, and Hunt (1985); Sawford (1986), and Thomson (1987) should follow. Robinson (1965) is easy to read and explains the mathematical reasons why we should follow Thomson (1987) in using Gaussian forcing. Sawford (1985) is a good review of the theory and the practical problems of applying the Langevin model. 


\section{Postscript}

One pioneering publication (Durbin, 1983) is not cited in the preceding text because the only copy available at that time of writing was almost completely illegible. This Postscript was written about three years later when a better copy was obtained. What follows is a synopsis of Durbin's (1983) contribution of 69 pages.

After the Introduction, Durbin reviews the Wiener process and continuous Markov processes in terms of a stochastic differential equation for a random walk (random displacements) in Lagrangian coordinates. The Fokker-Planck equation is introduced as the Eulerian equivalent of the Lagrangian stochastic differential equation. The Ito calculus for integrating a stochastic differential equation is explained. This leads into a discussion of numerical integration of a stochastic differential equation for random displacements and criteria for various boundary conditions. The discussion of boundary conditions is more extensive than in many other publications on stochastic models for turbulent diffusion.

Durbin then relates the stochastic model for random displacements to the Eulerian diffusion equation by means of the Fokker-Planck equation before discussing eddy diffusion in (1) shear flow. (2) the atmospheric surface layer, and (3) channel flow. The relations between turbulence statistics and the coefficient for eddy diffusivity are described.

The last section consists of a discussion of the Langevin equation for random velocity increments as a model for dispersion in both homogeneous and inhomogeneous turbulence. The comment is made in a footnote that "the issue lof proper formulation for inhomogeneous and nonstationary turbulence] is not finally resolved." The paper ends with a derivation of the asymptotic form (for increasing time) of the Langevin equation, the random displacement equation. 


\section{References}

Arnold, L., 1974: Stochastic differential equations: Theory and applications. John Wiley and Sons, New York.

Chandrasekhar, S., 1943: Stochastic problems in physics and astronomy. Rev. Mod. Phys., 15, 1-89.

Durbin, P.A., 1983: Stochastic differential equations and turbulent dispersion. NASA Reference Publication 1103.

Feller, W., 1968: An introduction to probability theory and its applications, Vol. 1, 3rd ed. John Wiley and Sons, New York.

Feller, W., 1971: An introduction to probability theory and its applications, Vol. 2, 2nd ed. John Wiley and Sons, New York, 1971.

Gardiner, C.W., 1983: Handbook of stochastic methods. Springer-Verlag, Berlin.

Gifford, F.A., 1982: Horizontal diffusion in the atmosphere: A Lagrangian-dynamical theory. Atmos. Environ., 16, 505-512.

Hänggi, P., and P. Talkner, 1980: A remark on truncation schemes of cumulant hierarchies. J. Stat. Phys., 22, 65-67.

Hanna, S.R., 1979: Some statistics of Lagrangian and Eulerian wind fluctuations. J. Appl. Met., $18,518-525$.

Kolmogorov, A.N., 1968: Local structure of turbulence in an incompressible viscous fluid at very high Reynolds numbers. Dokl. Akad. Nauk. 30, 301-305 (1941). Reprinted in Sov. Phys. Usp., 10, 734-736.

Krasnoff, E., and R.L. Peskin, 1971: The Langevin model for turbulent diffusion. Geophys. Fl. Dynam., 2, 123-146.

Legg, B.J., and M.R. Raupach, 1982: Markov-chain simulation of particle diffusion in inhomogeneous flows: The mean drift velocity induced by a gradient in Eulerian velocity variance. Boundary-Layer Meteorol., 24, 3-13.

Lin, C.C., and W.H. Reid, 1963: Turbulent flow, theoretical aspects. Handbuch der Physik, VIII/2, 438-523.

Lukacs, E., 1960: Characteristic functions. Hafner Publishing Company, New York.

Lumley, J.L., 1962: The mathematical nature of the problem of relating Lagrangian and Eulerian statistical functions in turbulence. Mecanique de la Turbulence, CNRS, Paris.

Monin, A.S., and A.M. Yaglom, 1975: Statistical fluid mechanics: Mechanics of turbulence, Vol. 2. MIT Press, Cambridge.

Novikov, E.A., 1963: Random force method in turbulence theory. Sov. Phys. JETP, 17, 1449-1454.

Novikov, E.A., 1986: The Lagrangian-Eulerian probability relations and the random force method for nonhomogeneous turbulence. Phys. Fluids, 29, 3907-390৬. 
Obukhov, A.M., 1959: Description of turbulence in terms of Lagrangian variables. Adv. Geophys., 6, 113-116.

Papoulis, A., 1962: The Fourier integral and its applications. McGraw-Hill Book Company, Inc., New York.

Parzen, E., 1960: Modern probability theory and its applications. John Wiley and Sons, New York.

Pawula, R.F., 1967a: Generalizations and extensions of the Fokker-Planck-Kolmogorov equations. IEEE Trans. Inform. Theory 13, 33-41.

Pawula, R.F., 1967b: Approximation of the linear Boltzmann equation by the Fokker-Planck equation. Phys. Rev., 162, 186-188.

Robinson, D.W., 1965: A theorum concerning the positive metric. Commun. Math Phys., 1, 89-94.

Sawford, B.L., 1984: The basis for, and some limitations of the Langevin equation in atmospheric relative diffusion modelling. Atmos. Environ., 18, 2405-2411.

Sawford, B.L., 1985: Lagrangian statistical simulation of concentration mean and fluctuation fields. J. Clim. Appl. Meteorol., 24, 1152-1166.

Sawford, B.L., 1986: Generalized random forcing in random-walk turbulent diffusion models. Phys. Fluids., 29, 3582-3585.

Taylor, G.I., 1921: Diffusion by continuous movements, Proc. Lond. Math. Soc. Ser. 2, 20, 196-211.

Thomson, D.J., 1984: Random walk modelling of diffusion in inhomogeneous turbulence. Quart. J. R. Met. Soc., 110, 1107-1120.

Thomson, D.J., 1987: Criteria for the selection of stochastic models of particle trajectories in turbulent flows. J. Fluid Mech., 180, 529-556.

Uhlenbeck, G.E., and L.S. Ornstein, 1930: On the theory of the Brownian motion. Phys. Rev., 36, 823-841.

van Dop, H., F.T.M. Nieuwstadt, and J.C.R. Hunt, 1985: Random walk models for particle displacements in inhomogeneous unsteady turbulent flows. Phys. Fluids, 28, 1639-1653.

van Kampen, N.G., 1981: Stochastic processes in physics and chemistry. North-Holland Publishing Company, Amsterdam.

Wilson, J.D., B.J. Legg, and D.J. Thomson, 1983: Calculation of particle trajectories in the presence of a gradient in turbulent-velocity variance. Boundary-Layer Meteorol., 27, 163-169.

Wilson, J.D., G.W. Thurtell, and G.E. Kidd, 1981: Numerical simulation of particle trajectories in inhomogeneous turbulence, II: Systems with variable turbulence velocity scale. Boundary-Layer Meteorol., 21, 423-441. 


\section{Two "Relatives" of the Langevin Equation}

\section{The Markov or Diffusion Equation Limit}

Durbin (1984) stated that one of the constraints on a consistently formulated Langevin model is that it must reduce to an eddy diffusion model as the correlation time scale $\tau_{L} \rightarrow 0$ (or as $t / \tau_{L} \rightarrow \infty$ ). He showed that the Langevin model for inhomogeneous turbulence proposed by Wilson, Thurtell, and Kidd (1981) and modified by Wilson, Legg, and Thomson (1983), the WTK/WLT model, meets that requirement. (Durbin did not explicitly identify it as the WTK/WLT model.) Equation (48) in van Dop, Nieuwstadt, and Hunt (1985) is presented as the Markov limit of a Langevin model that, for stationary inhomogeneous turbulence, is that of Legg and Raupach (1982), the LR model. It can be shown that the Markov limit of van Dop et al., is, for stationary turbulence, identical to the diffusion equation limit obtained by Durbin. deBaas and Troen (1989) presented derivations of the Markov limits of the WTK/WLT and LR models and showed them to be equal. These Markov or diffusion equation limits are equivalent to the random displacement model for turbulent diffusion proposed by Boughton, Delaurentis, and Dunn (1987). They gave a different demonstration of the relation between the Langevin equation and its Markov limit, one that is similar to the derivation on pp. 134-136 of Arnold (1974) of the Ornstein-Uhlenbeck-process for Brownian motion. We will follow deBaas and Troen, for the most part, in the following.

We begin with Eq. (27) for the WTK/WLT model,

$$
d\left(\frac{w}{\sigma_{w}}\right)=-\frac{w d t}{\sigma_{w} \tau_{L}}+\left(\frac{2}{\tau_{L}}\right)^{1 / 2} d W(t)+\frac{d \sigma_{w}}{d z} d t
$$

and Eq. (31a) for the LR model,

$$
d w=-\frac{w d t}{\tau_{L}}+\sigma_{w}\left(\frac{2}{\tau_{L}}\right)^{1 / 2} d W(t)+\frac{d \sigma_{w}^{2}}{d z} d t .
$$

We follow deBaas and Troen in using the scaling relations

$$
\tau_{L}=\alpha T
$$

and

$$
\sigma_{w}=\beta S,
$$

keeping $S$ and $T$ constant. Then Eqs. (71a-b) become, respectively,

$$
d\left(\frac{w}{\beta S}\right)=-\frac{w d t}{\alpha \beta S T}+\left(\frac{2}{\alpha T}\right)^{1 / 2} d W(t)+\beta \frac{d S}{d z} d t
$$

and

$$
d w=-\frac{w d t}{\alpha T}+\beta S\left(\frac{2}{\alpha T}\right)^{1 / 2} d W(t)+\beta^{2} \frac{d S^{2}}{d z} d t
$$


we get the following by first integrating and then multiplying by $\alpha \beta$ and $\alpha$, respectively:

$$
\alpha\left[\left(\frac{w}{S}\right)_{\imath}-\left(\frac{w}{S}\right)_{0}\right]=\int_{0}^{t}\left[-\frac{w d t}{S T}+\left(\frac{2 \alpha \beta^{2}}{T}\right)^{1 / 2} d W(t)+\alpha \beta^{2} \frac{d S}{d z} d t\right]
$$

and

$$
\alpha\left(w_{t}-w_{o}\right)=\int_{0}^{t}\left[-\frac{w d t}{T}+\left(\frac{2 \alpha \beta^{2} S^{2}}{T}\right)^{1 / 2} d W(t)+\alpha \beta^{2} \frac{d S^{2}}{d z} d t\right] .
$$

deBaas and Troen stated that cert::n constraints must be met in order that the Markov or diffusion equation limits with $\tau_{L} \rightarrow 0$ ( $t$ fixed) and $t \rightarrow \infty\left(\tau_{L}\right.$ fixed) be equivalent. They (and in a similar manner both Arnold and Boughton et al., ) required that

$$
\alpha \rightarrow 0 \text { and } \beta \rightarrow \infty \text { such that } \alpha \beta^{2}=\sigma_{w}^{2} \tau_{L}=K=\text { a constant } .
$$

Then, as stated in Durbin as well as in deBaas and Troen, the left sides of Eqs. (74 a-b) are bounded for all time in a "mean square" sense and approach zero as $\alpha \rightarrow 0$. Related material is given in Arnold pp. 12-14 and Gardiner (1983) pp. 40 and 83-84. We let

$$
d z=w d t
$$

and combine Eqs. (74 a-b) with Eqs. (75) and (76) to get

$$
\int_{z=}^{z} \frac{d z}{S T}=\int_{0}^{t}\left[\left(\frac{2 K}{T}\right)^{1 / 2} d W(t)+K \frac{d S}{d z} d t\right]
$$

and

$$
\int_{z_{0}}^{z} \frac{d z}{T}=\int_{0}^{t}\left[\left(\frac{2 S^{2} K}{T}\right)^{1 / 2} d W(t)+K \frac{d S^{2}}{d z} d t\right]
$$

We follow Durbin and deBaas-Troen in using the rules of the Ito calculus (Arnold, pp. 88-99: Gardiner, pp. 88-96) to differentiate the left side of Eqs. (77a-b). This involves the use of the first two terms of the Taylor's series,

$$
f(x)-f(a)=(x-a) f^{\prime}(a)+\frac{(x-a)^{2}}{2 !} f^{\prime \prime}(a)+\ldots,
$$

where $(x-a) f^{\prime}(a)$ corresponds to $d z / S T$ in Eq. (77a) and $d z / T$ in Eq. (77b). Then we get the following:

$$
\frac{d z}{S T}-\frac{1}{2}\left(\frac{d z}{S T}\right)^{2} \frac{d(S T)}{d z}=\left(\frac{2 K}{T}\right)^{1 / 2} d W(t)+K \frac{d S}{d z} d t
$$

for the WTK/WLT model and

$$
\frac{d z}{T}-\frac{1}{2}\left(\frac{d z}{T}\right)^{2} \frac{d T}{d z}=\left(\frac{2 S^{2} K}{T}\right)^{1 / 2} d W(t)+K \frac{d S^{2}}{d z} d t
$$


for the LR model. We rewrite the above as follows:

$$
d z-\frac{(d z)^{2}}{2 S T} \frac{d(S T)}{d z}=\left(2 K S^{2} T\right)^{1 / 2} d W(t)+K S T \frac{d S}{d z} d t
$$

and

$$
d z-\frac{(d z)^{2}}{2 T} \frac{d T}{d z}=\left(2 K S^{2} T\right)^{1 / 2} d W(t)+K T \frac{d S^{2}}{d z} d t .
$$

We use Eqs. (72a-b) and (75) to get Eqs. (80a-b) in terms of $\sigma_{w}$ and $\tau_{L}$ :

$$
\frac{(d z)^{2}}{2 \sigma_{w} \tau_{L}} \frac{d\left(\sigma_{w} \tau_{L}\right)}{d z}-d z+\left(2 \sigma_{w}^{2} \tau_{L}\right)^{1 / 2} d W(t)+\sigma_{w} \tau_{L} \frac{d \sigma_{w}}{d z} d t=0
$$

and

$$
\frac{(d z)^{2}}{2 \tau_{L}} \frac{d \tau_{L}}{d z}-d z+\left(2 \sigma_{w}^{2} \tau_{L}\right)^{1 / 2} d W(t)+\tau_{L} \frac{d \sigma_{w}^{2}}{d z} d t=0
$$

We let

$$
\begin{aligned}
& A_{a}=\frac{1}{2 \sigma_{w} \tau_{L}} \frac{d\left(\sigma_{w} \tau_{L}\right)}{d z}=\frac{1}{2}\left[\frac{1}{\tau_{L}} \frac{d \tau_{L}}{d z}+\frac{1}{\sigma_{w}} \frac{d \sigma_{w}}{d z}\right] \\
& A_{b}=\frac{1}{2 \tau_{L}} \frac{d \tau_{L}}{d z}, \\
& B=-1, \\
& C=D W(t)+E d t \\
& D=\left(2 \sigma_{w}^{2} \tau_{L}\right)^{1 / 2}, \\
& E_{a}=\sigma_{w} \tau_{L} \frac{d \sigma_{w}}{d z}, \\
& E_{b}=\tau_{L} \frac{d \sigma_{w}^{2}}{d z}=2 \sigma_{\omega} \tau_{L} \frac{d \sigma_{w}}{d z} .
\end{aligned}
$$

Equations (81a-b) in combination with Eq. (82) give a quadratic equation in $d z$ :

$$
A(d z)^{2}+B(d z)+C=0 .
$$

The solutions are

$$
d z=\frac{-B \pm\left(B^{2}-4 A C\right)^{1 / 2}}{2 A} .
$$

With $B=-1$ from Eq. (82), we select the root

$$
d z=\frac{1-(1-4 A C)^{1 / 2}}{2 A}
$$

We assume that $(1-4 A C)^{1 / 2}$ can be approximated by the truncated series

$$
(1-4 A C)^{1 / 2}=1-\frac{4 A C}{2}-\frac{(4 A C)^{2}}{8}-\ldots
$$

Then from Eqs. (85) and (86),

$$
d z=C+A C^{2}+\ldots
$$


Then Eqs. (82) and (87) give

$$
d z=D d W(t)+E d t+A\left[D^{2}(d W(t))^{2}+2 D E d W(t) d t+E^{2}(d t)^{2}\right] .
$$

Per Ito's calculus (Arnold, p. 91; Gardiner, p. 89),

$$
\begin{aligned}
(d t)^{2} & =0, \\
d W(t) d t & =0 \\
(d W(t))^{2} & =d t
\end{aligned} .
$$

Then Eq. (88) reduces to

$$
d z=\left(E+A D^{2}\right) d t+D d W(t) .
$$

From Eqs. (82) and (90) for the WTK/WLT model:

$$
d z=\left(\sigma_{w} \tau_{L} \frac{d \sigma_{w}}{d z}+\sigma_{w} \frac{d\left(\sigma_{w} \tau_{L}\right)}{d z}\right) d t+\left(2 \sigma_{w}^{2} \tau_{L}\right)^{1 / 2} d W(t)
$$

Similarly for the LR model:

$$
d z=\left(\tau_{L} \frac{d \sigma_{w}^{2}}{d z}+\sigma_{w}^{2} \frac{d \tau_{L}}{d z}\right) d t+\left(2 \sigma_{w}^{2} \tau_{L}\right)^{1 / 2} d W(t)
$$

Equations (91a-b) reduce to the same equation:

$$
d z=\frac{d\left(\sigma_{w}^{2} \tau_{L}\right)}{d z} d t+\left(2 \sigma_{w}^{2} \tau_{L}\right)^{1 / 2} d W(t) \quad !
$$

This is consistent with Eq. (10) in Durbin (1984); Eqs. (34), (35), and (48) of van Dop et al. (1985) for stationary inhomogeneous turbulence; and Eqs. (22) and (34) of deBaas and Troen (1989). If we let (Sawford, 1985), Table 1; van Dop et al., Eq. (50))

$$
K=\sigma_{w}^{2} \tau_{L}
$$

we get Eq. (92) in the same form as Eq. (4) in Durbin and the combination of Eqs. (3), (7a) with $w_{s}(t)=0$, and (7b) of Boughton et al., (1987):

$$
d z=\frac{d K}{d z} d t+(2 K)^{1 / 2} d W(t)
$$

We have noted that the Langevin equation, e.g., Eqs. (71a-b), is applicable for $\tau_{k} \ll t$. Boughton et al., and deBaas and Troen stated that the Markov limit equation, Eq. (92), is valid for $t \gg \tau_{L}$. deBaas and Troen also made the comment that $\tau_{i}$, is a time scale related to the inhomogeneity of the turbulence is also relevant, and they assumed in their development that $\tau_{i} \gg \tau_{L}$.

This closing paragraph is analogous to the preceding section titled "Postscript" in that it was written long after the above on the Markov limit of the Langevin equation. Durbin (1980) presented results for both the Langevin and random displacement models for turbulent dispersion, without describing the mathematical process that transforms the first model into the second. Durbin and Hunt (1980) also presented results from both models and likewise omitted the derivation of the second from the first. However, Durbin (1983) presented such a derivation. 


\section{The Fokker-Planck Equation: Introduction}

We return to the Fokker-Planck equation which we mentioned briefly in the Introduction to these Notes. It was originally developed as an alternate to the Langevin model of Brownian motion. Obukhov (1959) made the pioneering suggestion that the Fokker-Planck equation be used as a model for turbulent diffusion. However, according to the available literature, those who followed his suggestion during the 1960's and into the early 1980's chose to use the Langevin equation instead. The following from Gardiner (1983) p. viii probably explains why:

"It is true that there is nothing in a stochastic differential equation that is not in a FokkerPlanck equation, but the stochastic differential equation is so much easier to write down and manipulate that only an excessively zealous purist would try to eschew the technique. On the other hand, only similar purists of an opposing camp would try to develop the theory without the Fokker-Planck equation ..."

This apparent neglect of the Fokker-Planck equation ended when van Dop, Nieuwstadt, and Hunt (1985) used it as an aid in their analysis of the Langevin model for turbulent diffusion. Sawford (1986) and Thomson (1987) made similar use of the Fokker-Planck equation. These authors took advantage of the different perspectives given by these two equations as explained by van Kampen (1981) p. 209,

"The Fokker-Planck equation is a special type of master equation, which is often used as an approximation to the actual one. The Langevin equation is a different but mathematically equivalent formulation."

and by Arnold (1974) p. 156,

"...the solutions of stochastic differential equations and diffusion processes represent essentially the same classes of processes despite their completely different definitions" (emphasis in original).

The following quotations from van Dop et al. are pertinent to our study of the Fokker-Planck equation:

"...the Eulerian equivalent of the Langevin equation ... [is] the Fokker-Planck equation,"

"A stochastic process is Markovian when its future state depends only on its present state (and some transition rule),"

"The Markovian property is essential when we want to derive from the Langevin equation an expression which describes the evalution of the probability density corresponding with the process, the 'Fokker-Planck' equation" (emphasis in original),

"In the derivation of the Fokker-Planck equation it is assumed that this random forcing is Gaussian," 
"Its derivation is standard, though not quite trivial" (emphasis added).

We copy two equations from van Dop et al: their Eq. (18), the Langevin equation,

$$
d w=\left(-w \tau_{L}^{-1}+a_{1}\right) d t+a_{2}^{1 / 2} d W(t)
$$

and their Eq. (22), the Fokker-Planck equation,

$$
\frac{\partial P}{\partial t}+w \frac{\partial P}{\partial z}=\frac{\partial}{\partial w}\left[\left(\frac{w}{\tau_{L}}-a_{1}\right) P\right]+\frac{1}{2} a_{2} \frac{\partial^{2} P}{\partial w^{2}} .
$$

Here $a_{i}=a_{i}(z, t), \tau_{L}=\tau_{L}(z, t)$, and the probability density $P=P(z, w, t)$. The latter is the joint probability that a particle is located in the interval $(z, z+d z)$ and in the velocity range $(w, w+d w)$. We compare Eq. (65a) for the WTK/WLT model of Wilson, Thurtell, and Kidd (1981) that was modified by Wilson, Legg, and Thomson (1983) to get

$$
\begin{gathered}
a_{1}=\sigma_{w}\left[\left(\frac{w}{\sigma_{w}}\right)^{2}+1\right] \frac{\partial \sigma_{w}}{\partial z} \\
a_{2}=2 \sigma_{w}{ }^{2} / \tau_{L} .
\end{gathered}
$$

Here $a_{i}=a_{i}(z)$ and $\tau_{L}=\tau_{L}(z)$ because the WTK/WLT model was developed for stationary homogeneous turbulence. We recall from the development in Eqs. (35)-(58) that $a_{1}$ and $a_{2}$ in Eqs. (95) and (97a-b) are equal to the first and second moments, respectively, of the random forcing function associated with $W(t)$. We will find that $a_{1}$ and $a_{2}$ in Eq. (96) are equal to the first and second moments of the random forcing function defined in another way. For the present, we ake the word of Arnold, Gardiner, van Dop et al., and van Kampen that $a_{1}$ and $a_{2}$ in Eq. (95) are respectively equal to $a_{1}$ and $a_{2}$ in Eq. (96).

We have made the following physical interpretations of the terms in Eq. (95), the Langevin equation:

$w \tau_{L}^{-1}$ represents the "fading memory" of the particle velocity,

$a_{1}$ is the "drift correction" in inhomogeneous turbulence, and

$a_{2}$ is the variance of the amplitude of the random forcing if the covariance of the random increments $d W(t)$ is a Dirac delta function.

van Kampen makes the following comments on p. 208:

"The first term on the right of [Eq. (96)] has been called "transport term', 'convective term', or 'drift term'; the second one 'diffusion term' or 'fluctuation term'. Of course these names should not prejudge their physical interpretation."

In view of the meanings given in the case of the Langevin equation for turbulent diffusion, we make the following interpretations for the corresponding Fokker-Planck model:

$$
w \tau_{L}^{-1} \text { is a "transport" term, }
$$


$w \tau_{L}^{-1}$ is a "transport" term,

$a_{1}$ is a "drift" term, and

$a_{2}$ is a "fluctuation" term.

However, "diffusion" is more generally used than "fluctuation" in the literature and we see below that "diffusion" is more appropriate for the Fokker-Planck equivalent of the Markov limit of the Langevin equation.

We found in the preceding section that the Markov limit of Eq. (95) in combination with Eqs. $(97 a-b)$ is (see Eqs. (93)-(94))

$$
d z=\frac{d K}{d z} d t+(2 K)^{1 / 2} d W(t)
$$

where

$$
K=\sigma_{w}^{2} \tau_{L}
$$

The corresponding Fokker-Planck equation is given by the combination of Eqs. (4) and (7) in Boughton, Delaurentis, and Dunn (1987) as

$$
\frac{\partial P}{\partial t}=-\frac{\grave{o}}{\partial z}\left(P \frac{\partial K}{\partial z}\right)+\frac{1}{2} \frac{\partial^{2}}{\partial z^{2}}(2 K P)
$$

where the first term on the right is the "drift" term and the second is the "diffusion" term. This equation reduces to the classic diffusion equation,

$$
\frac{\partial P}{\partial t}=\frac{\partial}{\partial z}\left(K \frac{\partial P}{\partial z}\right)
$$

which, fur stationary Gaussian turbulence, is equivalent to Eqs. (49) and (50) in van Dop et a!.

\section{The Fokker-Planck Equation: Derivation}

We have seen that the form of the Langevin equation comes from assuming a combination of deterministic and random processes with different characteristic times and that the coefficients in Eqs. (95) and (97) are equal to the first two moments of the random forcing function. How do we get the form and coefficients of the Fokker-Planck equation, Eq. (96)? If we begin with fundamentals, we must consider three processes and their equations in the following order:

the Markov process and the Chapman-Kolmogorov equation,

the jump process and the master equation, and

the diffusion process and the Fokker-Planck equation.

These three items are covered by Wang and Uhlenbeck (1945) in their Secs. 3, 5, and 8, respectively. They use the name "Smoluchowski" instead of the currently-accepted "Chapman-Kolmogorov." They treat the jump process and the master equation, but they do not use the terms "jump" and "master." Arnold (1974) discusses the first item in Secs. 2.1-2.2 and the third in Secs. 2.5-2.6, but 
he does not cover the second. van Kampen (1981) presents material on all three in Secs. IV.2, V. 1, and VIII. 2, respectively. He notes on p. 82 that some have used the name "Smoluchowski" for the Chapman-Kolmogorov equation, but he avoids that name because "it is used indiscriminantly for several closely related but not identical equations." He describes the master equation as a differential or difference form of the Chapman-Kolmogorov equation. Gardiner (1983) covers these three items in Secs. 3.2.1, 3.5.1, and 3.5.2, respectively. However his Eq. (3.4.22) in Sec. 3.4.1 that he calls the "differential Chapman-Kolmogorov equation" is not to be confused with van Kampen's description of the master equation. Gardiner reduces his Eq. (3.4.22) to the master equation in Sec. 3.5.1 and to the Fokker-Planck equation in Sec. 3.5.2. We found van Kampen to be the easiest to understand among these four references, so the following is based largely on his material.

We begin, of necessity, by defining the process formulated in 1906 by Markov that is now named after him. It can be defined in words as "the 'future' is independent of the 'past' when we know the 'present'" and "the past and future are statistically independent when the present is known" (Arnold, pp. 27 and 29). The Markov process is defined by two probability functions: the probability density

$$
P_{1}\left(y_{1}, t_{1}\right) \geq 0
$$

for the process to be in state $y$, at time $t_{1}$, and the transition probability

$$
P_{1 \mid 1}\left(y_{2}, t_{2} \mid y_{1}, t_{1}\right) \geq 0
$$

for the change from $y_{1}$ at $t_{1}$ to $y_{2}$ at $t_{2}$. The times in the Markov process are ordered as

$$
t_{1}<t_{2}<t_{3} \text {, etc. }
$$

Equation (102) is the basis of the parenthetical remark in the above quotation from van Dop. Nieuwstadt, and Hunt (1985): "A stochastic process is Markovian when its future state depends only on its present state (and some transition rule)." The probability functions Eqs. (101)-(102) cannot be arbitrary but must follow the following two constraints if the process is to be Markovian. First, the probability density of $y_{2}$ at time $t_{2}$ is equal to the integral over all values of $y_{1}$ of the product of the probability density Eq. (101) and the transition probability Eq. (102):

$$
P_{1}\left(y_{2}, t_{2}\right)=\int P_{1 \mid 1}\left(y_{2}, t_{2} \mid y_{1}, t_{1}\right) P_{1}\left(y_{1}, t_{1}\right) d y_{1} \text {. }
$$

Second, a process starting at time $t_{1}$ from a value $y_{1}$ reaches a value $y_{3}$ at time $t_{3}$ by way of any of the possible values of $y_{2}$ at time $t_{2}$ (van Kampen, p. 83):

$$
P_{1 \mid 1}\left(y_{3}, t_{3} \mid y_{1}, t_{1}\right)=\int P_{1 \mid 1}\left(y_{3}, t_{3} \mid y_{2}, t_{2}\right) P_{1 \mid 1}\left(y_{2}, t_{2} \mid y_{1}, t_{1}\right) d y_{2}
$$

In other words, this condition for a Markov process statistically decouples the process value $y_{3}$ at time $t_{3}$ (the future) from its value $y_{1}$ at time $t_{1}$ (the past) because, for these values of $y_{1}$ and $y_{3}$, there is a wide range of possible values for $y_{2}$ at time $t_{2}$ (the present). Equation (105) is the Chapman-Kolmogorov equation.

Our next step in the derivation of the Fokker-Planck equation involves Markov processes whose transition properly depends on the time difference alone, not on the successive times $t_{1}$, and $t_{2}$. From Eq. (102) for the transition probability,

$$
P_{1 \mid 1}\left(y_{2}, t_{2} \mid y_{1}, t_{1}\right)=T_{\tau}\left(y_{2} \mid y_{1}\right)
$$


where

$$
\tau=t_{2}-t_{1}
$$

The Chapman-Kolmogorov equation, Eq. (105), becomes

$$
T_{\tau+\tau^{\prime}}\left(y_{3} \mid y_{1}\right)=\int T_{\tau}^{\prime}\left(y_{3} \mid y_{2}\right) T_{\tau}\left(y_{2} \mid y_{1}\right) d y_{2}
$$

with

$$
\tau, \tau^{\prime}>0
$$

The above four relations are given on p. 87 of van Kampen for stationary Markov processes, but he notes on p. 92 that nonstationary Markov processes can be homogeneous in time with transition probabilities dependent on the time difference alone.

We now come to a difficult part of the derivation. It involves the behavior of the ChapmanKolmogorov equation for $T_{\tau+\tau}^{\prime}$ as $\tau^{\prime} \rightarrow 0$. van Kampen gives the following on p. 101 for the transition probability $T_{\tau}^{\prime}$ for small values of $\tau^{\prime}$ :

$$
T_{\tau}^{\prime}\left(y_{2} \mid y_{1}\right)=\left(1-a_{o} \tau^{\prime}\right) \delta\left(y_{2}-y_{1}\right)+\tau^{\prime} W\left(y_{2} \mid y_{1}\right)+O\left(\tau^{\prime}\right)
$$

where $O(\tau) / \tau^{\prime} \rightarrow 0$ as $\tau^{\prime} \rightarrow 0 . W\left(y_{2} \mid y_{1}\right)$ is the transition probability per unit time for the process from $y_{1}$ to $y_{2}$ with

$$
W\left(y_{2} \mid y_{1}\right) \geq 0
$$

and

$$
a_{o}\left(y_{1}\right)=\int W\left(y_{2} \mid y_{1}\right) d y_{2}
$$

van Kampen states on pp. 101-102 that Eq. (110) is adopted in his book as generally valid. van Kampen's Eq. (V.1.1), our Eq. (110), is based on his Eq. (IV.6.8) in Sec. IV.6 on the process of radioactive decay. Similar material on the Poisson process, a Markov process used to model radioactive decay, is given in Sec. XVII in Feller (1968), and his Eq. (XVII.2.5) is equivalent to our Eq. (110). His explanation on pp. 447-448 of that equation is more clear than that of van Kampen. The first term on the right is the probability that the process is in state $y_{2}$ at the beginning of the interval $\tau^{\prime}$ and stays there. The second term on the right is the probability that the process jumps from $y_{1}$ to $y_{2}$ during $\tau^{\prime}$. The third term, $O\left(\tau^{\prime}\right)$, is the much smaller probability that the process will make more than one jump during $\tau^{\prime}$ to get to $y_{2}$. We have noted above that $O(\tau) / \tau^{\prime} \rightarrow 0$ and $\tau^{\prime} \rightarrow 0$. Equation (110) has been clearly known for a long time because it, with different notation, is on p. 272 of the still-in-print classic by Doob (1953). It appears in all three references (Doob, Feller, van Kampen) that Eq. (110) is "assembled" on the basis of mathematical reasoning, not explicitly derived from another equation.

van Kampen apparently continues by changing from $\left(y_{2} \mid y_{1}\right)$ to $\left(y_{3} \mid y_{2}\right)$ in Eq. (110) and combining the result with Eq. (108), the Chapman-Kolmogorov equation, to get

$$
\begin{aligned}
T_{\tau+\tau^{\prime}}\left(y_{3} \mid y_{1}\right)= & \int\left[1-a_{o}\left(y_{3}\right) \tau^{\prime}\right] \delta\left(y_{3}-y_{2}\right) T_{\tau}\left(y_{2} \mid y_{1}\right) d y_{2} \\
& +\int \tau^{\prime} W\left(y_{3} \mid y_{2}\right) T_{\tau}\left(y_{2} \mid y_{1}\right) d y_{2} \\
& +\int O\left(\tau^{\prime}\right) T_{\tau}\left(y_{2} \mid y_{1}\right) d y_{2} .
\end{aligned}
$$


It appears that van Kampen lets $\delta\left(y_{3}-y_{2}\right)=T_{\tau^{\prime} \rightarrow 0}\left(y_{3} \mid y_{2}\right)$ so

$$
\int\left[1-a_{o}\left(y_{3}\right) \tau^{\prime}\right] \delta\left(y_{3}-y_{2}\right) T_{\tau}\left(y_{2} \mid y_{1}\right) d y_{2}=\left[1-a_{o}\left(y_{3}\right) \tau^{\prime}\right] T_{\tau}\left(y_{3} \mid y_{1}\right)
$$

From Eqs. (113)-(114) we get

$$
\begin{aligned}
T_{\tau+\tau^{\prime}}\left(y_{3} \mid y_{1}\right)= & {\left[1-a_{o}\left(y_{3}\right) \tau^{\prime}\right] T_{\tau}\left(y_{3} \mid y_{1}\right) } \\
& +\int \tau^{\prime} W\left(y_{3} \mid y_{2}\right) T_{\tau}\left(y_{2} \mid y_{1}\right) d y_{2} \\
& +\int O\left(\tau^{\prime}\right) T_{\tau}\left(y_{2} \mid y_{1}\right) d y_{2}
\end{aligned}
$$

This is identical, except for the last term on the right which will be dropped, to the top unnumbered equation on p. 102 in van Kampen. We change $y_{1}$ to $y_{3}$ in Eq. (112) and substitute the result into Eq. (115) to get

$$
\begin{aligned}
& T_{\tau+\tau^{\prime}}\left(y_{3} \mid y_{1}\right)-T_{\tau}\left(y_{3} \mid y_{1}\right)=-\int \tau^{\prime} W\left(y_{2} \mid y_{3}\right) T_{\tau}\left(y_{3} \mid y_{1}\right) d y_{2} \\
& \quad+\int \tau^{\prime} W\left(y_{3} \mid y_{2}\right) T_{\tau}\left(y_{2} \mid y_{1}\right) d y_{2}+\int O\left(\tau^{\prime}\right) T_{\tau}\left(y_{2} \mid y_{1}\right) d y_{2}
\end{aligned}
$$

We divide by $\tau^{\prime}$ and let $\tau^{\prime} \rightarrow 0$ to get van Kampen's Eq. (V.1.4):

$$
\frac{\partial}{\partial t} T_{\tau}\left(y_{3} \mid y_{1}\right)=\int\left[W\left(y_{3} \mid y_{2}\right) T_{\tau}\left(y_{2} \mid y_{1}\right)-W\left(y_{2} \mid y_{3}\right) T_{\tau}\left(y_{3} \mid y_{1}\right) d y_{2}\right.
$$

This is the master equation. van Kampen continues on p. 102 with a reference back to Sec. IV.4 that the transition probability " $T_{r}\left(y_{2} \mid y_{1}\right)$ is identical with the distribution function $P . *\left(y_{2}\right)$ of the subensemble determined by the initial value $y_{1}$." (This is proven in Sec. 9.2 of Arnold.) Then van Kampen rewrites Eq. (117) as

$$
\frac{\partial P(y, t)}{\partial t}=\int\left[W\left(y \mid y^{\prime}\right) P\left(y^{\prime}, t\right)-W\left(y^{\prime} \mid y\right) P(y, t)\right] d y^{\prime}
$$

He also writes it in difference form (which we will not copy) and makes the comment that its meaning becomes particularly clear in that form: it is a gain-less equation for the probability of each state $y^{\prime}$. In other words, it is a balance equation for jumps into and out of a given state.

We now follow pp. 214-215 of van Kampen for the last part of our derivation of the FokkerPlanck equation. We first redefine the transition probability per unit time $\mathrm{W}$, as a function of the size $r$ of the jump and the starting point $y^{\prime}$ :

$$
W\left(y \mid y^{\prime}\right)=W\left(y^{\prime} ; r\right)
$$

with

$$
r=y-y^{\prime} .
$$

Then the master equation, Eq. (118), can be rewritten as

$$
\frac{\partial P(y, t)}{\partial t}=\int W\left(y_{r} ; r\right) P(y-r, t) d r-P(y, t) \int W(y ;-r) d r
$$


We assume that only small jumps occur so $W\left(y^{\prime} ; r\right)$ is a sharply peaked function of $r$ but varies slowly with $y^{\prime}$ :

$$
\begin{aligned}
W\left(y^{\prime} ; r\right) & \approx 0 \text { for }|r|>\delta, \\
W\left(y^{\prime}+\Delta y ; r\right) & \approx W\left(y^{\prime} ; r\right) \text { for }|\Delta y| \delta .
\end{aligned}
$$

We also assume that $P(y, t)$ varies slowly with $y$ in the same sense as $W\left(y^{\prime} ; r\right)$ in Eq. (122b). These assumptions make it possible to expand the product $[W(y-r ; r) P(y-r, t)]$ in a Taylor's series:

$$
\begin{aligned}
W(y-r ; r) & P(y-r, t)=W(y ; r) P(y, t) \\
& -r \frac{\partial}{\partial y}[W(y ; r) P(y, t)]+\frac{r^{2}}{2} \frac{\partial^{2}}{\partial y^{2}}[W(y ; r) P(y, t)] .
\end{aligned}
$$

Then we combine Eqs. (121) and (123) to get

$$
\begin{aligned}
\frac{\partial P(y, t)}{\partial t}=\int & W(y ; r) P(y, t) d r \\
& -\int r \frac{\partial}{\partial y}[W(y ; r) P(y, t)] d r \\
& +\frac{1}{2} \int r^{2} \frac{\partial^{2}}{\partial y^{2}}[W(y ; r) P(y, t)] d r \\
& -P(y, t) \int W(y ;-r) d r .
\end{aligned}
$$

The first and last terms on the right cancel. In analogy to Eq. (112), we let

$$
a_{\nu}(y)=\int_{-\infty}^{\infty} r^{\nu} W(y ; r) d r
$$

Then we get the Fokker-Planck equation,

$$
\frac{\partial P(y, t)}{\partial t}=-\frac{\partial}{\partial y}\left[a_{1}(y) P\right]+\frac{1}{2} \frac{\partial^{2}}{\partial y^{2}}\left[a_{2}(y) P\right]
$$

which is Eq. (V.1.5) on p. 102 in van Kampen.

Now that we have it, of what use is it? We take our answer from pp. 211-212 of van Kampen. The main use of the Fokker-Planck equation is an approximate description of Markov processes $y(t)$ with small steps. The word "approximate" means that the Fokker-Planck equation, Eq. (126). is an approximation to the master equation, Eq. (118). The Fokker-Planck equation has two features that make it easier to use than the master equation: (1) it is a partial differential equation instead of differentio-integral equation, and (2) it does not require specification of the kernel $W\left(y \mid y^{\prime}\right)$ but the much simpler task of determining the moments $a_{1}$ and $a_{2}$ per Eq. (125). 


\section{The Fokker-Planck Equation: Relation to the Langevin Equation}

In the preceding section, we started with the probability density, transition probability, and Chapman-Kolmogorov equation that define the Markov process. We used these to derive the master equation, which van Kampen describes as a differential form of the Chapman-Kolmogorov equation, for the jump process. We concluded by deriving the Fokker-Planck equation from the master equation. We now address the question, "What is the relation between the Fokker-Planck and Langevin equations?"

The relation between these equations is discussed in Sec. 9 of Wang and Uhlenbeck (1945), Sec. VI.3 in Doob (1953), Secs. 9.3-9.4 in Arnold (1974), Sec. VIII.8 in van Kampen, and Secs. 4.3.3-4.3.4 in Gardiner (1983). Of these, the treatment by Wang and Uhlenbeck is brief and perhaps too simple, those by Doob and Arnold are lengthy and highly mathematical, that by Gardiner is brief but rather mathematical, and that by van Kampen on pp. 239-246 appears to be the best for our purposes. The common theme of all these references is that the coefficients $a_{1}$ and $a_{2}$ that appear in the Langevin and Fokker-Planck equations (see Eqs. (95) and (96) for example) are identical. That is, the first two moments of the random forcing function associated with the process $d W(t)$ in the Langevin equation are respectively equal to the first two moments of jump transition probability $W(y ; r)$ in the Fokker-Planck equation.

van Kampen begins by showing that, if $a_{1}$ and $a_{2}$ are constants and the forcing function is Gaussian (the Fokker-Planck equation is Gaussian by definition and we found in the first part of these notes that Gaussian forcing is required for the Langevin equation), the coefficients $a_{1}$ and $a_{2}$ are identical in the two equations. He finishes with the nonlinear case in which $a_{1}=a_{1}(y)$ and $a_{2}=a_{2}(y)$ to give the "generally nonlinear" Langevin equation,

$$
d y=a_{1}(y) d t+\left[a_{2}(y)\right]^{1 / 2} d W(t)
$$

He states that Eq. (127) "as it stands has no meaning." This is because $d W(t)$ is a random forcing function that results in jumps in $y(t)$. With Eq. (127) alone, neither the value of $y$ when a pulse arrives or the appropriate value of $y$ for determing $a_{2}$ is known. If Ito's rule (that the value of $y$ before the jump is used to determine the value of $a_{2}$ ) is followed, then Eq. (127), the Langevin equation is equivalent to Eq. (126), the Fokker-Planck equation. van Kampen cites Doob, presumably Sec. VI.3 on pp. 273-291, for proof of this equivalence. In the Appendix on p. 629 in Doob, major credit for the results presented in Sec. VI.3 is given to Kolmogorov, Feller and Ito. (Actually, van Kampen's Eq. (8.15) on p. 244 is for $d y / d t$ instead of $d y$ and the forcing function is white noise $\xi(t)$ instead of the incremental Wiener process $d W(t)$.)

\section{References}

Arnold, L., 1974: Stochastic differential equations: Theory and applications. John Wiley and Sons, New York.

Boughton, B.A., J.M. Delaurentis, and W.E. Dunn, 1987: A stochastic model of particle diffusion in the atmosphere. Boundary-Layer Meteorol., 40, 147-163.

deBaas, A.F., and I. Troen, 1989: A stochastic equation for diffusion in inhomogeneous conditions. Physica Scripta, 40, 64-72. 
Doob, J.L., 1953: Stochastic processes. John Wiley and Sons, New York.

Durbin, P.A., 1980: A random fight model of inhomogeneous turbulent dispersion. Phys. Fluids 23, 2151-2153.

Durbin, P.A., 1983: Stochastic differential equations and turbulent dispersion. NASA Reference Publication 1103.

Durbin, P.A., 1984: Comments on paper by Wilson et al. (1981) and Legg and Raupach (1982). Boundary-Layer Meteorol., 29, 409-411.

Durbin, P.A., and J.C.R. Hunt 1984: Dispersion from elevated sources in turbulent boundary layers. J. de Mechanique, 19, 679-695.

Feller, W., An introduction to probability theory and its application, Vol. 1. John Wiley and Sons, New York, 1968.

Gardiner, C.W., 1983: Handbook of stochastic methods. Springer-Verlag, Berlin.

Legg, B.J., and M.R. Raupach, 1982: Markov-chain simulation of particle diffusion in inhomogeneous flows: The mean drift velocity induced by a gradient in Eulerian velocity variance. Boundary-Layer Meteorol., 24, 3-13.

Obukhov, A.M., 1959: Description of turbulence in terms of Lagrangian variables. Adv. Geophys., 6, 113-116.

Sawford, B.L., 1986: Generalized random forcing in random-walk turbulent diffusion models. Phys. Fluids, 29, 3585.

Thomson, D.J., 1987: Criteria for the selection of stochastic models of particle trajectories in turbulent flows. J. Fluid Mech., 180, 529-556.

van Dop, H., F.T.M. Nieuwstadt, and J.C.R. Hunt, 1985: Random walk models for particle displacements in inhomogeneous unsteady turbulent flows. Phys. Fluids, 28, 1639-1653.

van Kampen, N.G., 1981: Stochastic processes in physics and chemistry. North-Holland Publishing Company, Amsterdam.

Wang, M.C., and G.E. Uhlenbeck, 1945: On the theory of the Brownian motion II. Rev. Mod. Phys., 17, 323-342.

Wilson, J.D., B.J. Legg, and D.J. Thomson, 1983: Calculation of particle trajectories in the presence of a gradient in turbulent-velocity variance. Boundary-Layer Meteorol., 27, 163-169.

Wilson, J.D., G.W. Thurtell, and G.E. Kidd, 1981: Numerical simulation of particle trajectories in inhomogeneous turbulence II: Systems with variable turbulence velocity scale. Boundary-Layer Meteorol., 21, 423-441. 


\section{Present Applications of the Langevin Model}

\section{Introduction}

The preceding emphasized the physical basis and mathematical theory of the Langevin model for diffusion in turbulent flows. We now turn to applications of this model to diffusion in the atmospheric boundary layer. The available literature indicates that the development of the Langevin model is relatively "mature" for vertical diffusion in those regimes for which the atmospheric turbulence is well-characterized by proven scaling relations. One is the surface layer and the other is the convective boundary layer.

We will study selected publications on applications of the Langevin model to turbulent diffusion in the surface and convective boundary layers. As a preface to the discussion of each group of papers, we will review the applicable theory for each case: neutral surface layer, stable and unstable surface layers, and (the unstable) convective boundary layer. It is assumed in almost all of the application papers that the turbulence is stationary and horizontally homogeneous; these conditions are used in the theoretical models of each turbulent boundary layer considered. The exceptions are two papers in which the Langevin model is applied to the surface layer over complex terrain.

We will discover in these applications papers how the parameterizations of the input parameters like the time scale $\tau_{L}$ and the variance $\sigma_{w}^{2}$ of the vertical velocity fluctuations evolved as the authors of successive papers improved on the results of earlier workers. We will give special attention to the parameterization of the timescale $\tau_{L}$ in inhomogeneous turbulence (in which it does not have a precise definition).

\section{The Surface Boundary Layer}

The simplest inhomogeneous case of turbulence is the constant-stress region in the neutral boundary layer in which the turbulence is horizonally homogeneous but vertically inhomogeneous (Sawford, 1985). An introductory account of this case is given on pp. 135-143 and 150-156 of Townsend (1976) and a more detailed discussion is presented on pp. 257-295 of Monin and Yaglom (1971). From p. 138 of Townsend for the neutral constant-stress region outside the thin viscous sublayer,

$$
\begin{gathered}
-<u w>_{0}=u_{*}^{2}, \\
\varepsilon=u_{*}^{3} / k z
\end{gathered}
$$

and

$$
\partial U / \partial z=u_{*} / k z
$$

Here $u$ and $w$ are, respectively, the longitudinal and vertical velocity fluctuations, $\left\langle u w>_{0}\right.$ is the Reynolds-averaged turbulent kinematic shear stress at the surface, $u_{*}$ is the friction velocity, $\varepsilon$ is the rate of turbulent kinetic energy dissipation, $k$ as the van Karman constant for momentum $(k \approx 0.4), z$ is the height above the surface, and $U$ is the mean longitudinal velocity. We note from Fig. 5.2 and the related discussion on pp. 135-139 of Townsend that the vertical (called "lateral" in Townsend) diffusion of turbulent kinetic energy is assumed to be negligible in the constant-stress 
layer. In addition, the product of Eqs. (128) and (130) is equal to Eq. (129). Therefore, under these conditions, the production rate of turbulent kinetic energy $-\langle u w\rangle \partial U / \partial z$ is equal to its dissipation rate $\varepsilon$.

We will use the Reynolds analogy between the turbulent diffusion of momentum and that of a scalar and write a companion to Eq. (130):

$$
\partial \Theta / \partial z=\theta_{*} / k_{\Theta} z
$$

where

$$
\theta_{*}=-<w \theta>_{0} / u_{*} .
$$

Here $\theta$ is the mean value of the scalar, $\theta$ is the turbulent fluctuation of the scalar, and $k_{\theta}$ is the von Karman constant for the scalar $\left(k_{\theta} \approx 0.4\right.$, but $k_{\theta}$ is not necessarily equal to $k$ ). The eddy diffusivity model is valid in the constant-stress region so we can use Eqs. (128) and (130)-(132) to write (Monin and Yaglom, pp. 262-264).

$$
-<u w>_{0}=K \partial U / \partial z
$$

and

$$
-<w \theta>_{0}=K_{\theta} \partial \Theta / \partial z
$$

where

$$
K=k u_{*} z
$$

and

$$
K_{\theta}=k_{\theta} u . z .
$$

The scalar in the following application is the mass concentration of a material species, the "marked" particles of the Langevin model.

The Langevin equation for diffusion in the neutral constant-stress layer has the same form as that for homogeneous turbulence, Eq. (22),

$$
d w=-\left(w / \tau_{L}\right) d t+\sigma_{w}\left(2 / \tau_{L}\right)^{1 / 2} d W(t)
$$

where $\tau_{L}$ is the time scale, $\sigma_{w}$ is the standard deviation of $w, t$ is time, and $d W(t)$ is a Wiener process. In the constant-stress layer, the length and time scales of the turbulence increase linearly with height but the velocity scale $\sigma_{w}$ is constant according to a relation given by Sawford (1985):

$$
\sigma_{w}=b_{1} u \text {. }
$$

where $b_{1}$ is a constant and

$$
\tau_{L}=b_{2} z(t) / \sigma_{w}
$$

where $b_{2}$ is another constant. The values of these constants are determined from physical and numerical experiments. Sawford reviewed several papers in which Eqs. (135)-(137) on their equivalents were used to model diffusion in this neutral surface layer. Most of these authors used a value for $b_{1}$ of about 1.3, and they also required that the Langevin equation match the Markov or diffusion equation limit (see Eqs. (71a)-(94) and the related discussion). This requirement. in combination with many of Eqs. (128)-(137), gives

$$
b_{1} b_{2}=K_{\theta}(z) / u_{*} z=\sigma_{w}^{2} \tau_{L} / u_{*} z=k_{\theta} .
$$


Sawford found that Ley (1982), of those papers reviewed, was probably the most successful in matching Langevin simulations to both experimental data and theoretical solutions.

Ley applied Eqs. (135)-(138) to "the diffusion of a passive pollutant over level terrain in the surface layer of a neutral boundary layer." The mean wind profile for these conditions is given by the integral of Eq. (130), the familiar logarithmic relation

$$
U(z)=\left(u_{*} / k\right) \ln \left(z / z_{0}\right)
$$

Where $z_{0}$ is the surface roughness height. Ley modeled the turbulent fluctuations of both the longitudinal and vertical velocities, but we will consider only the vertical fluctuations. She used the recommended values of $b=1.3$ and got the best matches to experimental data, Lagrangian similarity theory, and solutions of the diffusion equation with values of $k=0.41$ and $k_{\theta}=0.39$. The corresponding value of $b_{2}$ in Eq. (137) is, from Eq. (138), 0.3.

The next case in order of increased complexity is the diabatic surface layer described by MoninObukhov scaling (Calder, 1966; Monin and Yaglom, 1971, pp. 417-430; Townsend, 1976, pp. 375378). Townsend gives a brief summary, Monin and Yaglom present a more detailed discussion, and Calder gives an excellent introductory explanation. The diabatic surface layer is characterized by both constant shear stress (like the neutral surface layer) and constant vertical heat flux (which is equal to zero in the neutral layer). The following equations for the neutral surface layer are applicable without change to the diabatic surface layer: Eqs. (128), (129), (132), and (133a-b) where the symbol $\theta$ now represents temperature as well as mass concentration of a species. We expand the definition of $\theta_{*}$ in Eq. (132) as follows:

$$
\theta_{*}=-<w \theta>_{0} / u_{*}=-H_{0} / \rho_{0} C_{p} u_{*}
$$

where $H_{0}$ is the surface heat flux, $p_{0}$ is the air density, and $C_{p}$ is the specific heat at constant pressure. The other equations for the neutral surface layer (Eqs. (130), (131), and (134a-b)) are made applicable to the diabatic surface layer by means of a simple modification:

$$
\begin{gathered}
\partial U / \partial z=u_{*} \phi(z / L) / k z_{1} \\
\partial \theta / \partial z=\theta_{*} \phi_{\theta}(z / L) / k_{\theta} z_{\theta} \\
K=k_{*} z / \phi(z / L), \\
K_{\theta}=k_{\theta} u_{*} z / \phi_{\theta}(z / L),
\end{gathered}
$$

where $\phi$ and $\phi_{\theta}$ are universal non-dimensional functions that are equal to unity if the surface layer is neutral. The parameter $L$ is the Monin-Obukhov scale length which we will define and discuss below.

We will approach the Monin-Obukhov scale length in two ways. First, as stated in Monin and Yaglom and, with greater clarity, in Calder whom we quote:

"... for the region of fully turbulent flow in the horizonally homogeneous surface layer the vertical gradients of mean velocity and temperature are uniquely determined by just the three parameters $\left[f / T, u_{*}\right.$, and $\left.\theta_{*}\right] "$

where the symbols in the brackets are ours, not those used by Calder. The friction velocity $u_{0}$ is related to the turbulent shear stress or the vertical turbulent diffusion of momentum. The scalar 
parameter $\theta_{*}$ is, in this case, the ratio of the vertical temperature flux to the friction velocity. Finally, $g / T$ is a buoyancy parameter assuming an ideal gas with $g$ representing the acceleration of gravity and $T$ the absolute air temperature. These three parameters can be combined to form the Monin-Obukhov length scale:

$$
L=U_{*}^{2} T / k g \theta_{*}=-u_{*}^{3} \rho_{0} C_{p} T / k g H_{0}
$$

The parameter $L$ is more convenient to use than the quantity $g / T$; furthermore in stable conditions $L>0$ and $H_{0}$ is negative (downward heat flux, cooling of surface layer) and in unstable conditions $L<0$ and $H_{0}$ is positive (upward heat flux, warming of surface layer), Monin and Yaglom (p. 427) note that the van Karman constant $k$ was introduced into the definition of $L$ by Obukhov in 1946 and that its use has been preserved by tradition. Calder comments that it is introduced solely as a matter of convenience.

The above is based on dimensional analysis; our second approach emphasizes the physics and follows van Ulden and Holtslag (1985). The product of the three parameters $g / T, u_{*}$, and $\theta_{*}$ is, from Eq. (140),

$$
B=-g u_{*} \theta_{*} / T=g H_{0} / \rho_{0} C_{p} T
$$

where $B$ is the rate of buoyancy production $\left(H_{0}>0\right)$ or destruction $\left(H_{0}<0\right)$ of turbulent kinetic energy. For the neutral surface layer $\left(H_{0}=0\right)$, we have noted that Eq. (129) is the equation for both the production (by shear stress) and the dissipation (by viscosity) of turbulent kinetic energy. We rewrite Eq. (129) as

$$
S=u_{*}^{3} / k z
$$

where $S$ is the shear-stress production rate of turbulent kinetic energy. Then the ratio of the rates of buoyant to shear-stress production of turbulent kinetic energy is, from Eqs. (143)-(145),

$$
B / S=-k g z \theta_{*} / u_{*}^{2} T=-z / L
$$

This shows that the dimensionless ratio $-z / L$ is an indicator of the relative importance of buoyancy and shear stress in the turbulent boundary layer. The effect of buoyancy approaches zero as $|z / L| \rightarrow O$, and the effect of shear stress becomes negligible for sufficiently large values of $|z / L|$. Unlike the derivation of Eq. (143) for $L$ based on dimensional analysis, this approach introduces the use of the von Karman constant $k$ in a logical way via Eq. (145). We now have two questions:

What are the ranges of values for $z$ and $z / L$ for which Monin-Obukhov scaling is valid?

What are the forms and values of the functions $\phi(z / L)$ and $\phi_{\theta}(z / L)$ in Eqs. (141a)$(142 \mathrm{~b}) ?$

The answers to the first question are relatively straight forward. According to Townsend (pp. 139-143), Eqs. (128)-(130) and (139) for the neutral boundary layer are applicable for $z \gg z_{0}$ where $z_{0}$ is the surface roughness length. We will also apply this rule to the diabatic surface layer. Holtslag and Nieuwstadt (1986) define the (fuzzy) boundaries between the different scaling regimes. some well-established and others poorly understood, of the idealized atmospheric boundary layer. The surface layer, with its Monin-Obukhov scaling, is the bottom tenth of the turbulent boundary layer $(z / h<0.1$ where $h$ is the height of the turbulent flow) and is bounded by the stability limits 
$z / L=-1$ and $h / L=10$. In physical terms, these limits mean that the effect of the surface shear stress is at least equal to, if not greater than, the effect of the buoyancy forces.

It is more difficult to answer the second question about the values and forms of the function $\phi(z / L)$ and $\phi_{\theta}(z / L)$. As noted in the lengthy discussion on pp. 430-456 of Monin and Yaglom, there is no definitive theory for these functions and they are based on empirical fits to experimental data. Many investigators have proposed about as many different relations. Yaglom (1977) wrote one of the most recent surveys of this situation and commented, "... the existing experimental data are apparently insufficient for the reliable determination of the universal functions entering fluxprofile relationships." He noted several contributing factors. First, the values of the von Karman constants in Eqs. (141a)-(142b) are known from experiments "with rather low precision," and different workers have usf $d$ values ranging from 0.35 to 0.435 . If the data for $\phi(z / L)$ and $\phi_{\theta}(z / L)$ are plotted using a common value (e.g., $k=k_{\theta}=0.4$ ), there is still significant scatter. Yaglom discussed several probable causes: instrument errors (e.g., different instruments deployed on joint experiments have given quite different results), uncertainty about the averaging times required for reliable determination of turbulent fuxes and other parameters, and the idealized conditions assumed for Monin-Obukhov theory are never strictly satisfied. However, there appears to be general agreement on the following about $\phi(z / L)$ and $\phi_{\theta}(z / L)$ :

These functions approach a value of unity as stability conditions are changed from either stable or unstable to neutral $\left(\phi(z / L) \rightarrow 1\right.$ and $\phi_{\theta}(z / L) \rightarrow 1$ as $|z / L| \rightarrow 0$,

These functions are significantly different for stable $(z / L>0)$ and unstable $(z / L<0)$ conditions,

The function for momentum flux $\phi(z / L)$ is different from that for scalar flux $\phi_{\theta}(z / L)$.

We will defer recommending specific formulas for these functions and return to applications of the Langevin model to the surface laver.

Ley and Thomson (1983) extended Ley's (1982) Langevin model to diabatic surface layers over level homogeneous terrain. They independently used heuristic arguments to obtain the LR drift correction (Legg and Raupach, 1982) and used the finite-difference equivalent of Eq. (31b),

$$
d w=\left(-\frac{w}{\tau_{L}}+2 \sigma_{w} \frac{d \sigma_{w}}{d z}\right) d t+\sigma_{w}\left(\frac{2}{\tau_{L}}\right)^{1 / 2} d W(t)
$$

They were aware of the WTK drift correction (Wilson, Thurtell, and Kidd, 1981) and showed in an appendix that the two corrections are equivalent if the turbulence is only slightly inhomogeneous (see our comparison of Eqs. (30b) and (31b)). Ley and Thomson used Monin-Obukhov theory: Eqs. (141a) and (142b) together with empirical relations to $\phi(z / L)$ and $\phi_{\theta}(z / L)$, the former for the mean wind profile and the latter in combination with the diffusion equation or Markov limit relation,

$$
K_{\theta}=\sigma_{w}^{2} \tau_{L}
$$

to get values for $\tau_{L}$ for use in Eq. (147). They used empirical relations based on field experiments to get height-dependent values for $\sigma_{w}$ and $d \sigma_{w} / d z$ for use in Eqs. (147)-(148). Their results are in good agreement with two sets of concentration data from diffusion experiments, one on the Kansas 
prairie in 1956 and the other on Porton Down in southern England in 1962-1963. Ley (1982) used neutral-condition data from these experiments for comparison with her results.

We have not found any subsequent published applications of the Langevin equation to diffusion in the surface layer over flat ground. Thomson, in the Introduction to his 1986 paper stated that the ability of the Langevin model to simulate diffusion uder these conditions was well established. The subject of Thomson (1986) is the application of the Langevin model to diffusion in the complex three-dimensional surface layer over rough terrain. He compared his results with data from diffusion conditions under approximately neutral conditions with westerly and easterly winds across an asymmetrical NNW-SSE valley in South Wales. His results were encouraging, but he found that the complex mean-flow pattern was fully as important as the turbulent diffusion in determining the concentration patterns.

Thomson began with a review of his 1984 work in which he showed that, for Gaussian turbulence, Eq. (147) requires a specific non-Gaussian forcing function while the WTK model as reformulated by WLT (Wilson, Legg, and Thomson, 1983), Eq. (30b),

$$
d w=\left\{-\frac{w}{\tau_{L}}+\sigma_{w}\left[\left(\frac{w}{\sigma_{w}}\right)^{2}+1\right] \frac{d \sigma_{w}}{d z}\right\} d t+\sigma_{w}\left(\frac{2}{\tau_{L}}\right)^{1 / 2} d W(t)
$$

requires Gaussian forcing. He had no experimental evidence for use in deciding whether Eq. (147) or (149) was the better model so he used the finite-difference version of the latter because Gaussian random numbers are easier to generate. (At that time he apparently was not aware that his rigorous solution for the non-Gaussian forcing required for Eq. (147) - see our Eqs. (61a-d)-represented a nonrealizable probability distribution.) Thomson used an available numerical solution for the steady three-dimensional flow over and in a somewhat idealized valley. His height-dependent values for the velocity variance $\sigma_{w}{ }^{2}$ and time scale $\tau_{L}$ were based on turbulence measurements at the site.

We found only one other application of the Langevin model to diffusion in the surface layer over complex terrain. Yamada (Yamada and Bunker, 1988) developed a two-part numerical model for transport and diffusion of pollutants over rugged terrain. The first part is a three-dimensional hydrodynamic model which calculates the mean velocity field and the standard deviations of the turbulent velocity fluctuations. These quantities are, in turn, used by the second part of the model which includes the equivalent of the Legg and Raupach (1982) version of the Langevin model (see Eq. (147)). Selected fixed values for the time scale $\tau_{L}$ in three dimensions are used. Yamada and Bunker commented that more study is needed on the turbulence scales of airflow-over complex terrain. They used this model to simulate one data set from the 1982 ASCOT experiment at Brush Creek, Colorado. They selected a period during the early morning when the surface inversion broke up, the nocturnal drainage flow stopped, and the wind reversed direction. They showed that differential warming of the canyon walls by the rising sun had a major effect on the mean flow field and, therefore, on the pollutant concentration profiles. This is consistent with the above finding of Thomson (1986) that it is necessary to simulate correctly both the mean flow field and the turbulent diffusion over complex terrain.

\section{The Convective Boundary Layer}

Like the surface layer, the unstable convective boundary layer (CBL) has been extensively studied theoretically and experimentally. The greatest recent advances in Langevin model development 
have been made in applying this model to the CBL. We will begin with a brief description of the $\mathrm{CBL}$ and then note the principal contributional of several papers in which the Langevin model is applied to the CBL.

According to the review by Holtslag and Nieuwstadt (1986), the CBL (there and in other references called the "mixed layer") is in the broad mid-portion of the turbulent atmospheric boundary layer $(0.1<z / h<0.8)$ under significantly unstable conditions $(h / L<-10)$ where $z$ is the elevation, $h$ is the height of the turbulent flow, and $L$ is the Monin-Obukhov scaling length. The above two numerical ranges combine to give $z / L<-1$. Therefore the CBL exists at higher elevations and under more unstable conditions than the surface layer. For the surface layer, the friction velocity $u$. (a function of the surface shear stress), the vertical temperature flux $\langle w \theta\rangle$, and the buoyancy parameter $g / \mathrm{T}$ are combined to define the Monin-Obukhov scaling length $L$ (see Eqs. (140) and (143)). The effects of the surface shear stress are negligible in the CBL but those of the temperature flux and the buoyancy parameter are not. Therefore $u_{*}$ and $L$ are not appropriate for scaling the CBL. Instead the turbulent boundary layer height $h$ is taken as the CBL scaling length and, in combination with the temperature flux and the buoyancy parameter, is used to define the velocity scale for the CBL:

$$
w_{*}=(h<w \theta>0 g / T)^{1 / 3}=(B h)^{1 / 3}
$$

where $B$ is defined by Eqs. (140) and (144).

The probability distribution function (PDF) of the vertical velocity fluctuations in the CBL is of special importance in applying the Langevin model. The PDF of the CBL is non-Gaussian because of the organized motion consisting of updrafts and downdrafts. The PDF has a negative mode and a positive tail such that the third moment (skewness) is positive. This is because the updrafts have higher velocities but occupy less area than the downdrafts with lower velocities.

Baerentsen and Berkowicz (1984), generally referred to hereafter as BB, made the valuable contribution of approximating the observed non-Gaussian PDF of the CBL with the sum of two Gaussian distributions, one for the updrafts and the other for the downdrafts. They required that certain relations for the zeroth through third moments of the PDF be satisfied. This technique was used by de Baas et al. (1986), Sawford and Guest (1987), Luhar and Britter (1989), and Weil (1990).

BB constructed a skewed PDF from the sum of two Gaussian distributions as follows:

$$
P_{a}(w \mid z)=\frac{1}{\sqrt{2 \pi}}\left\{\frac{\lambda_{1}}{\sigma_{1}} \exp \left[-\frac{1}{2}\left(\frac{w-m_{1}}{\sigma_{1}}\right)^{2}\right]+\frac{\lambda_{2}}{\sigma_{2}} \exp \left[-\frac{1}{2}\left(\frac{w-m_{2}}{\sigma_{2}}\right)^{2}\right]\right\}
$$

where $\lambda_{1}$ and $\lambda_{2}$ are the respective probabilities of occurrence of updrafts and downdrafts. They may also be interpreted as the respective areas occupied by updrafts and downdrafts. Here with $i=1,2$ (no summation), the mean velocity is

$$
m_{i}=<w_{i}>
$$

and the standard deviation of the velocity is

$$
\sigma_{i}=\left(\left\langle w_{i}^{2}\right\rangle\right)^{1 / 2}
$$


The parameters $\lambda, m$, and $\sigma$ are functions of $z$, the elevation. For a given value of $z, P_{\mathrm{a}}(w \mid z)$ is defined by six parameters: $\lambda_{1}, \lambda_{2}, m_{1}, m_{2}, \sigma_{1}, \sigma_{2}$. We note that $m_{1}>0$ (updraft) and $m_{2}<0$ (downdraft). BB equated the zeroth through third moments of $P_{a}$,

$$
<w^{n}>=\int_{-\infty}^{\infty} w^{n} P_{a}(w \mid z) d w,
$$

(with $n=0$ to 3 ) to the corresponding moments specified for the PDF of the CBL. Evaluation of these moments using Eqs. (151)-(153) gives the following:

$$
\begin{gathered}
\lambda_{1}+\lambda_{2}=1, \\
\lambda_{1} m_{1}+\lambda_{2} m_{2}=0, \\
\lambda_{1}\left(m_{1}^{2}+\sigma_{1}^{2}\right)+\lambda_{2}\left(m_{2}^{2}+\sigma_{2}^{2}\right)=\sigma_{w}^{2}, \\
\lambda_{1}\left(m_{1}^{3}+3 m_{1} \sigma_{1}^{2}\right)+\lambda_{2}\left(m_{2}^{3}+3 m_{2} \sigma_{2}^{2}\right)=<w^{3}>.
\end{gathered}
$$

We combine Eqs. (154a-b) to get solutions for $\lambda_{1}$ and $\lambda_{2}$ :

$$
\begin{aligned}
& \lambda_{1}=m_{2}\left(m_{2}-m_{1}\right)^{-1}, \\
& \lambda_{2}=-m_{1}\left(m_{2}-m_{1}\right)^{-1} .
\end{aligned}
$$

We follow Weil (1990) by introducing the ratio $R_{1}$

$$
R=\sigma_{1} / m_{1}=-\sigma_{2} / m_{2}
$$

and the skewness $S$,

$$
S=<w^{3}>/ \sigma_{w}^{3}
$$

(BB, deBaas et al., Sawford and Guest, and Luhar and Britter assumed $R=1$.) We use Eqs. (154cd) in combination with Eqs. $(155 \mathrm{a}-\mathrm{b})$ and $(156 \mathrm{a}-\mathrm{b})$ to give

$$
\begin{aligned}
& -m_{1} m_{2}\left(1+R^{2}\right)=\sigma_{w}{ }^{2} \\
& -m_{1} m_{2}\left(m_{1}+m_{2}\right)\left(1+3 R^{2}\right)=S \sigma_{w}^{3} .
\end{aligned}
$$

For convenience Weil introduces

$$
\begin{aligned}
& \propto=\left(1+R^{2}\right)\left(1+3 R^{2}\right)^{-1} \\
& \beta=1+R^{2} .
\end{aligned}
$$

Then Eqs. (154c') and (157b) give

$$
m_{1} m_{2}=-\sigma_{w}^{2} / \beta
$$

and Eqs. (154 $\left.\mathrm{c}^{\prime}-\mathrm{d}^{\prime}\right)$ and (157a) give

$$
m_{1}+m_{2}=\propto S \sigma_{w}
$$

We combine Eqs. $\left(154 c^{\prime \prime}-\mathrm{d}^{\prime \prime}\right)$ to get one quadratic equation in terms of $m_{1} / \sigma_{w}$,

$$
\left(\frac{m_{1}}{\sigma_{w}}\right)^{2}-\propto s\left(\frac{m_{1}}{\sigma_{w}}\right)-\frac{1}{\beta}=0
$$


and another in terms of $m_{2} / \sigma_{w}$,

$$
\left(\frac{m_{2}}{\sigma_{w}}\right)^{2}-\propto S\left(\frac{m_{2}}{\sigma_{w}}\right)-\frac{1}{\beta}=0 .
$$

Remembering that $m_{1}>0>m_{2}$, we get Weil's solutions:

$$
\begin{aligned}
& \frac{m_{1}}{\sigma_{w}}=\frac{1}{2}\left[\propto S+\left(\alpha^{2} S^{2}+\frac{4}{\beta}\right)^{1 / 2}\right], \\
& \frac{m_{2}}{\sigma_{w}}=\frac{1}{2}\left[\propto S-\left(\alpha^{2} S^{2}+\frac{4}{\beta}\right)^{1 / 2}\right] .
\end{aligned}
$$

With $R=1$ so $\propto=1 / 2$ and $B=2$, the above solutions are equivalent to those given by $\mathrm{BB}$ and by Luhar and Britter. This completes our solution for the non-Gaussian turbulence in the CBL in terms of $\mathrm{R}, \mathrm{S}$, and $\sigma_{w}$.

Baerentsen and Berkowicz (1984) used Gaussian forcing with two Langevin-type equations, one for the updrafts and the other for the downdrafts, so as to give an overall non-Gaussian PDF for the turbulence as described above. They chose to use the LR drift correction (Eq. (147)) instead of the WTK/WLT correction (Eq. (149)). They were apparently unaware of Thomson's (1984) work which showed that Gaussian forcing gives a Gaussian output only with the WTK/WLT correction. They claimed good agreement with experimental data for convection in water tanks.

deBaas et al. (1986) used the above solution of $\mathrm{BB}$ to define the first three moments of a non-Gaussian forcing function for a single Langevin equation. Their version of this equation for non-Gaussian inhomogeneous turbulence is equivalent to one developed by Thomson (1984)Sec. 3 whom they cited. The drift correction in this form of the equation reduces to that of LR (Eq. (147)) if the turbulence is Gaussian. As we have already noted, the corresponding non-Gaussian forcing function given by Thomson in 1984 is not realizable as he recognized in his 1987 paper. deBaas et al. did not mention the problem of realizability. deBaas et al. improved the CBL parameterization of $\mathrm{BB}$ and favorably compared their results with those of $\mathrm{BB}$ and data for experiments in water tanks, wind tunnels, and the field.

Sawford and Guest (1987) reviewed the work of BB and deBaas et al. and noted that the Langevin model of the latter is the better of the two. They observed that the deBaas et al. model is flawed in "that it is not asymptotic to a vertically homogeneous concentration for downstream, but rather, reduces to 0.7 times the homogeneous concentration in two thin layers along the top and bottom." Sawford and Guest took note that Thomson (1984) and Sawford (1986) had shown that (for Gaussian forcing and turbulence) the WTK/WLT drift correction (see Eq. (149)) gives a homogeneous asymptotic concentration so they used that correction. The CBL turbulence is non-Gaussian so they chose to use non-Gaussian forcing as did deBaas et al., but they were aware that a realizable non-Gaussian PDF has nonzero values for the third and higher moments. They stated that it is impractical to specify all the moments, and that only in rare circumstances are more than the first three moments of turbulence known. They used the above procedure of $\mathrm{BB}$ to define non-Gaussian PDFs (with known values for the first three moments) for both the initial turbulence field and the random forcing, realizing that they did not have an exact match between input and output. (We will find that Sawford was one of those who later solved the problem of consistent input and output for non-Gaussian turbulence.) They obtained better results than did 
$\mathrm{BB}$ and deBaas et al. Some of this improvement comes from the model and some from better turbulence parameterizations with more nearly correct boundary conditions at the bottom and (the more difficult) top.

The definitive Langevin model with Gaussian forcing that is consistent with a known output of non-Gaussian inhomogeneous turbulence was given independently by Luhar and Britter (1989) and Weil (1990). The BB procedure is used in both papers to define the first three moments of the non-Gaussian turbulence field. Both recognized the problems associated with non-Gaussian forcing and cited Thomson (1987). Both presented equivalent forms of the same model. Luhar and Britter present a complete derivation of this Langevin model; Weil gives the result and credits the derivation to Sawford who provided it in a private communication in 1987. The complete deriviation is lengthy and complex so we will give only a sketch of what is presented by Luhar and Britter.

We start with the Langevin equation

$$
d w=a(z, w) d t+b(z, w) d W(t)
$$

and its companion

$$
d z=w d t
$$

For stationary conditions the corresponding Fokker-Planck equation is

$$
w \frac{\partial P_{a}}{\partial z}+\frac{\partial}{\partial w}\left(a P_{a}\right)=\frac{1}{2} \frac{\partial^{2}}{\partial w^{2}}\left(b^{2} P_{a}\right)
$$

where $P_{a}=P_{a}(w, z)=P_{a}(w \mid z) P_{a}(z)$ where $P_{a}(z)=h^{-1}$ (the well-mixed condition) and $P_{a}(w \mid z)$ is defined by Eq. (151)(Weil, 1990). The above can be written in two parts as

$$
a P_{a}=\frac{\partial}{\partial w}\left(\frac{1}{2} b^{2} P_{a}\right)+\phi
$$

where $\phi$ satisfies

$$
\frac{\partial \phi}{\partial w}=-w \frac{\partial P_{a}}{\partial z}
$$

and $\phi \rightarrow 0$ as $|w| \rightarrow \infty$. We will simplify the PDF in Eq. (151) to the following for convenience:

$$
P_{a}(w \mid z)=\lambda_{1}(z) P_{1}(w \mid z)+\lambda_{2}(z) P_{2}(w \mid z) .
$$

We let

$$
b=\sigma_{w}\left(2 / \tau_{L}\right)^{1 / 2}
$$

as in the basic Langevin equation (Eq. (135)). We use Eq. (164) to rewrite one of the terms in Eq. (162a):

$$
\frac{\partial}{\partial w}\left(\frac{1}{2} b^{2} P_{a}\right)=-\frac{\sigma_{w}^{2}}{\tau_{L}} Q
$$

so

$$
a=\left(-\frac{\sigma_{w}^{2}}{\tau_{L}} Q+\phi\right) P_{a}^{-1}
$$


where

$$
Q=\frac{\lambda_{1}\left(w-m_{1}\right) P_{1}}{\sigma_{1}^{2}}+\frac{\lambda_{2}\left(w-m_{2}\right) P_{2}}{\sigma_{2}^{2}}
$$

and $\lambda_{1}, \lambda_{2}, m_{1}, m_{2}, \sigma_{1}, \sigma_{2}$ are as in Eqs. (151)-(159b). Then Eq. (160a) becomes

$$
d w=\left[\frac{-\left(\sigma_{w}^{2} / \tau_{L}\right) Q+\phi}{P_{a}}\right] d t+\sigma_{w}\left(\frac{2}{\tau_{L}}\right)^{1 / 2} d W(t)
$$

The random forcing is the Gaussian Wiener process with zero mean and variance $d t$. The solution for $\phi$ is

$$
\begin{array}{r}
\phi=\sigma_{i}{ }^{2} P_{i} \frac{d \lambda_{i}}{d z}+\frac{\lambda_{i} P_{i}}{2}\left(\frac{w^{2}}{\sigma_{i}^{2}}+1\right) \frac{d \sigma_{i}^{2}}{d z} \\
-\frac{1}{2} \frac{d}{d z}\left(\lambda_{i} m_{i}\right) \operatorname{erf}\left(\frac{w-m_{i}}{\sqrt{2} \sigma_{i}}\right)
\end{array}
$$

where $i=1,2$ (with summation). The above is the form given by Weil which is more compact than the equivalent in Luhar and Britter. Luhar and Britter explicitly state that Eqs. (168)-(169) reduce to Eq. (149) for inhomogeneous Gaussian turbulence and it is implied by Weil.

We now have three definitive forms of the Langevin model: Eq. (135) for the neutral surface layer, Eq. (149) for the diabatic surface layer, and Eqs. (168)-(169) for the CBL. The eddy diffusivity or $\mathrm{K}$-theory model is valid for the first two cases (providing $t \gg \tau_{L}$ ), but not for the CBL as demonstrated by Weil. We are not aware of any other Langevin models developed for other regimes such as the upper part of the boundary layer above the surface layer in near-neutral and stable conditions.

\section{Parameterization of the Time Scale}

We have noted that the time scale $\tau_{L}$ has an exact definition for only stationary, homogeneous turbulence (see Eqs. (14)-(18). This definition is approximate for weakly inhomogeneous or slightly nonstationary turbulence. Otherwise $\tau_{L}$ is loosely defined as a "local decorrelation time scale" (Sawford, 1985; Thomson, 1987). We will now review how $\tau_{L}$ was determined in the applications of the Langevin model to the surface and convective boundary layers that we reviewed in the two preceding sections.

Ley (1982) and Ley and Thomson (1983) used Eq. (134b) and (142b), respectively, in combination with Eq. (93) for the Markov or diffusion equation limit to determine the value for $\tau_{L}$. The more general relation is that for the diabatic surface layer studied by Ley and Thomson,

$$
\tau_{L}=k_{\theta} u_{*} z / \sigma_{w}^{2} \phi_{\theta}(z / L)
$$

For the neutral surface layer considered by Ley, $\phi_{\theta}(z / L)=1$ and Eq. (170) reduces to the relation she used. Here $k_{\theta}, u_{*}$, and $\sigma_{w}$ are constants for the neutral layer but $\sigma_{w}=\sigma_{w}(z)$ in the diabatic case.

For the surface layer over complex terrain, Thomson (1986) used empirical relations for $\tau_{L}=$ $\tau_{L}(z)$ based on turbulence measurements at the site while Yamada and Bunker (1988) used constant values for $\tau_{L}$. 
For the convective boundary layer (CBL), Baerentsen and Berkowicz (1984) used

$$
\tau_{i}=\sigma_{i}^{2} / \varepsilon
$$

to define a total of four different timescales associated with updrafts, downdrafts, and the transitive from one to another. Here $\varepsilon$ is the rate of dissipation of turbulent kinetic energy and $\sigma_{\varepsilon}$ is the standard deviation of turbulent velocity fluctuations. Subsequent workers did not choose to use such a complex scheme for the timescales. deBaas et al. (1986) used a constant value for $\tau_{L}$ :

$$
\tau_{L}=c h / w_{*}
$$

where $c$ is a dimensionless constant (after experimentation they chose $c=1, h$ is the height of the turbulent boundary layer, and $w_{*}$ is the CBL scale velocity given by Eq. (15). Sawford and Guest (1987) noted that Eq. (172) does not satisfy CBL similarity and chose an equation that does:

$$
\tau_{L}=\propto \sigma_{w}^{2} h / w_{*}^{3}
$$

They selected $\propto=2.5$ as a good fit to experimental data. They observed that Eq. (173) implies that $\varepsilon=$ constant throughout the CBL, but they did not give a relation for $\varepsilon$. Luhar and Britter (1989) also criticized Eq. (172), pointing out that it implies an unrealistic variation of $\varepsilon=\varepsilon(z)$. In addition, they noted that Eq. (173) implies that

$$
\varepsilon h / w_{*}^{3}=1 / \propto,
$$

which is a much better approximation to reality. However Luhar and Britter replaced the right side of Eq. (174) with an empirical function of $z / h$,

$$
\frac{\varepsilon h}{w_{*}^{3}}=1.5-1.2\left(\frac{z}{h}\right)^{1 / 3}
$$

which they showed to be an even better fit to the data. They used Eq. (150) for $w_{*}$ and Eq. (175) in combination with

$$
\tau_{L}=2 \sigma_{u}^{2} / C_{0} \varepsilon
$$

where $C_{0}$ is a universal constant to determine $\tau_{L}$, for use in Eq. (168). They let $C_{0}=2$ in most of their work; this reduces Eq. (176) to the same form as Eq. (171). They experimented with other values of $C_{0}$ and reported that the use of $C_{0}=5$ to 7 resulted in underprediction of maximum ground level concentrations from elevated sources in a CBL. Weil (1990) was apparently unaware of the work by Luhar and Britter and used Eq. (176) with $C_{0}=2$ and 3; the latter gave a better fit of Langevin model results to some Large Eddy Simulation (LES) data.

We will investigate the problem of the value of $C_{0}$ in the next section. In so doing, we will gain an understanding of the basis for Eq. (176). 


\section{The Lagrangian Structure Function and Its "Universal" Constant}

Luhar and Britter (1989) and Weil (1990) stated that $C_{0}$ in Eq. (176) is a universal constant from Kolmogorov's theory of locally isotropic turbulence in the inertial subrange and that it is related to the Lagrangian structure function. Both sources referred to Monin and Yaglom (1975) concerning the structure function and to Thomson (1987) for using $C_{0}$ in the Langevin model for scalar diffusion. Thomson similarly cited Monin and Yaglom for the structure function, but he credited van Dop et al. (1985) for originally relating $C_{0}$ to the Langevin diffusion model.

However, Pope (1983) may have priority in the use of $C_{0}$ in a different application of the Langevin model to turbulent flow: writing the Navier-Stokes equation for the incremental change of Lagrangian particle velocity as the sum of mean and fluctuating components. From Eqs. (10) and (11) of that paper,

$$
\begin{aligned}
& d \hat{U}_{i}=\left(\nu \nabla^{2}<U_{i}>-\frac{1}{\rho} \frac{\partial<\rho>}{\partial x_{i}}\right) d t \\
& +G_{i j}\left(\hat{U}_{j}-<U_{j}>\right) d t+\left(C_{0} \varepsilon\right)^{1 / 2} d W_{i}(t)
\end{aligned}
$$

where $\hat{U}$ is the Langrangian velocity, $U$ is the Eulerian velocity, $\nu$ is the kinematic viscosity, $\rho$ is the fluid density, $p$ is the pressure, $G_{i j}$ is a second-order tensor function of the local mean quantities, $C_{0}$ is the universal constant, $\varepsilon$ is the rate of dissipation of turbulent kinetic energy, and $d W_{i}(t)$ is an isotropic Wiener process. The first line on the right is the mean component of the velocity and the second line is the fluctuating component. The fluctuating component consists of the deterministic term $G_{i j}\left(\hat{U}_{j}-<U_{j}>\right) d t$ and the random term $\left(C_{0} \varepsilon\right)^{1 / 2} d W_{i}(t)$. Equation (177), without the mean flow term, has the same general form as the various Langevin equations that we have studied.

The Lagrangian structure function is defined as the ensemble average of the square of the change in Lagrangian velocity in the time interval $\Delta t$ (Monin and Yaglom, p. 83):

$$
D(\Delta t)=\left\langle w(t+\Delta t)-w(t)^{2}\right\rangle=\left\langle(\Delta w)^{2}>.\right.
$$

For Kolmogorov's inertial subrange $\tau_{k} \ll \Delta t \ll \tau_{L}$ where $\tau_{k}$ is the Kolmogorov time scale (Eq. (4)) and $\tau_{L}$ is the local Lagrangian decorrelation time (Monin and Yaglom, p. 359),

$$
D(\Delta t)=C_{0} \varepsilon \Delta t
$$

where $C_{0}$ and $\varepsilon$ are defined as for Eq. (177). We introduce a general Langevin equation,

$$
d w=a(z, w) d t+b(z, w) d W(t)
$$

where $d W(t)$ is an incremental Wiener process with zero mean and variance $d t$. We square both sides of Eq. (180) and take the ensemble average with $d t=\Delta t$ :

$$
<(\Delta w)^{2}>=<a^{2} d t^{2}+2 a b \Delta t \Delta w(t)+b^{2} \Delta t>=<b^{2}>\Delta t
$$

Similarly, from Eq. (177),

$$
<\left(\Delta \hat{U}_{i}\right)^{2}>=C_{0} \varepsilon \Delta t
$$


We combine Eqs. (178), (179), and (181a) to get

$$
<b^{2}>=C_{0} \varepsilon
$$

From Eq. (180) and other appearances of the Langevin equation going back to Eq. (22),

$$
<b^{2}>=2 \sigma_{w}^{2} / \tau_{L}
$$

We get Eq. (176) from Eqs. (182) and (183),

$$
\tau_{L}=2 \sigma_{w}^{2} / C_{0} \varepsilon
$$

The equivalent of the above derivation is in van Dop et al. (1985).

Monin and Yaglom (1975) did not give any values for $C_{0}$. Thomson (1987) cited Hanna's (1981) observations of Lagrangian (neutral balloon) trajectories in the atmosphere which indicate $C_{0}=4.0 \pm 2.0$ (Thomson's $C_{0}$ is Hanna's $2 \pi^{2} B$ ). van Dop et al. (1985) gave the equivalent of $C_{0}=1.6 \pm 0.6$ (their $2 \pi B=C_{0}$ ), but they did not identify the source of these values. Anand and Pope (1985) used the ideas of Pope (1983), including Eq. (177), to model the thermal wake behind a heated wire in grid turbulence and obtained $C_{0}=2.1$. Sawford and Guest (1988) were aware of this work by Anand and Pope, but they found that a value of $C_{0}$ of 5 to 10 in a Langevin model gave a much better fit to data for diffusion from a line source in the constant stress region of a neutral boundary layer. They ended their paper with the comment that these values of $C_{0}=2.1$ for grid turbulence and $C_{0}=5$ to 10 for the neutral surface layer " are clearly inconsistent with the concept of universality." However Pope and Chen (1990) returned to the data used by Anand and Pope and found that the results from a modified Langevin model suggested that $C_{0}$ is in the range of 2 to 5 . They tentatively specified $C_{0}=3.5$.

We have the means to calculate a value for $C_{0}$ in the neutral surface layer. We combine Eq. (184) with Eq. (138) to eliminate $\tau_{L}$ and get an equation for $\varepsilon$ which, in turn, we combine with Eq. (129) for $\varepsilon$. The results is

$$
C_{0}=2\left(k / k_{\theta}\right)\left(\sigma_{w} / u_{*}\right)^{4} .
$$

We set $k=k_{\theta}$ and use Eq. (136) with $b=1.3$ (see Ley (1982) and Sawford (1985)) to get $C_{0}=$ 5.7. This is within the 5 to 10 range mentioned by Sawford and Guest, and it is not significantly out of the 2 to 5 range given by Pope and Chen. However, the value of $C_{0}$ is still an open matter. An expanded version of the above has been published (Rodean, 1991).

\section{Other Turbulence Parameterizations}

"The quality of a diffusion model is strongly influenced by its meteorological input" which includes the vertical profiles of wind, temperature, and atmospheric turbulence (van Ulden and Holtslag, 1985). The first two profiles are important, but we are concerned here about the turbulence profile for the entire range of atmospheric conditions pertinent to diffusion modelling. Holtslag and Nieuwstadt (1986) reviewed the scaling of the different turbulence regimes in the atmospheric boundary layer and presented two diagrains illustrating these regimes for the unstable and stable boundary layers. They chose the dimensionless scale lengths $z / h$ and $h / L$ as the ordinate and abscissa, respectively, for their diagrams where $z$ is the elevation, above the surface, $h$ is the height 
of the turbulent boundary layer, and $L$ is the Monin-Obukhov scaling length defined by Eq. (143). They used logarithmic scales for the wide range of unstable conditions and linear scales for the more restricted range of turbulence in the stable boundary layer. Gryning et al. (1987) improved upon the two figures in Holtslag and Nieuwstadt by combining them as shown in Fig. 1. Note that logarithmic scales are used for the ordinate $z / h$ for both the unstable and unstable cases and for the abscissa $h /-L$ for the unstable case. However, the abscissa $h / L$ for the stable case is linear.

Seven different turbulent layers are identified in Fig. 1: surface, free convection, mixed (what we have called the "convective boundary layer" or "CBL"), entrainment, near-neutral upper, local scaling, z-less scaling, and intermittency. Gryning et al. applied various diffusion models (none based on the Langevin equation) to all but the entrainment and intermittency layers for which no scaling relations are known. They noted that their results included jumps of species concentration values across the borders between different turbulence regimes. They remarked in closing, "Improvements in the models proposed here may be introduced by continuous description of the vertical diffusion between the different scaling regions of the boundary layer." We have, in some preceding sections, studied applications of the Langevin model to the surface and mixed layers. We will now review height-dependent turbulence parameterizations used in these applications. Following Gryning et al. we will also consider the continuity (or otherwise) of turbulence parameterizations across the boundaries between the different turbulence regimes. We must remember that the boundaries in nature are fuzzy, not sharp like those in Fig. 1.

For the constant-stress region of the neutral surface layer $(h / L \approx 0)$, Ley $(1982)$ used

$$
\sigma_{w} / u_{*}=1.3
$$

where $\sigma_{w}$ is the standard deviation of the vertical velocity fluctuations and $u_{*}$ is the friction velocity. We will find that the above is a useful reference condition for other parameterizations.

For the unstable surface layer, Ley and Thomson (1983) chose the following from Panofsky et al. (1977):

$$
\sigma_{w} / u_{*}=1.3[1+(3 z /-L)]^{1 / 3}
$$

Panofsky et al. selected this empirical fit to data instead of another in order to meet two conditions. First, that Eq. (187) reduce to Eq. (186) as $(z /-L) \rightarrow 0$ Second, that Eq. (187) approach CBL scaling for large values of $(z /-L)$ :

$$
\sigma_{w} / u_{*} \sim(z /-L)^{1 / 3}
$$

We get Eq. (2) in Panofsky et al. from our Eqs. (140), (143), and (150):

$$
w_{*} / u_{*}=(h /-k L)^{1 / 3}
$$

where $w_{*}$ is the convective scaling velocity and $k$ is the von Karman constant.

We reviewed the parameterizations for $\sigma_{w}$ used by Baerentsen and Berkowicz (1984), de Baas et al. (1986) Sawford and Guest (1987), Luhar and Britter (1989), and Weil (1990). Of these, only Eqs. (36a-b) in Weil appear to offer the promise of compatibility with Eqs. (186) and (187):

$$
\frac{\sigma_{w}^{2}}{w_{*}^{2}}=\left[\frac{u_{*}^{3}}{w_{*}^{3}}\left(1.6-\frac{z}{h}\right)^{3 / 2}+1.2 \frac{z}{h}\left(1-0.98 \frac{z}{h}\right)^{3 / 2}\right]^{2 / 3}
$$




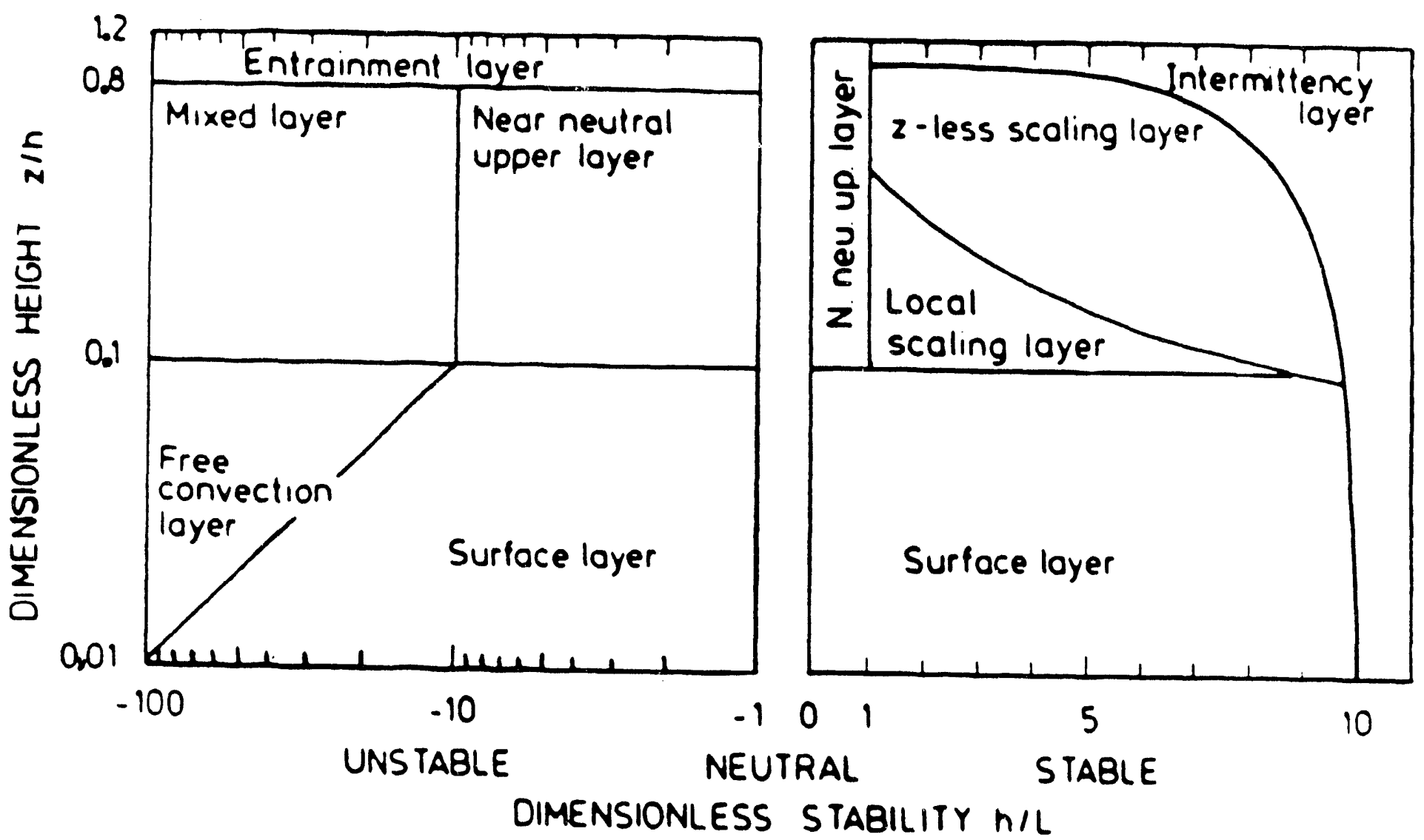

Fig. 1. The scaling regions of the atmospheric boundary layer, shown as function of the dimensionless height $z / h$, and the stability parameter $h / L$. A detailed discussion can be found in Holtslag and Nieuwstadt (1986). When used to determine diffusion regions, the dimensionless height is replaced by $z / h$, where $z$ is the source height. Reprinted by permission of Kluwer Academic Publishers and A.A.M. Holtslag. 
and

$$
\frac{<w^{3}>}{w^{3}}=0.84 \frac{z}{h}\left(1-\frac{z}{h}\right) 1
$$

where $\left\langle u^{3}\right\rangle$ is the third moment of the vertical velocity fluctuations in the CBL. Weil used the value 1.6 in Eq. (190a) to give " $\sigma_{w}=1.26 u_{*}$ at the surface in [approximate] agreement with Panofsky et al." If we change 1.6 to 1.69 , Eq. (190a) reduces to Eq. (186), exactly, as $z \rightarrow 0$. The Monin-Obukhov scaling length $L$ does not appear explicitly in any of the parameterizations for $\sigma_{w}$ for the CBL given by Baerentsen and Berkowicz through Weil, but $L$ is in there implicitly because of the relation between $u_{*}$ and $w$. given by Eq. (189).

We also need a parameterization for the rate of turbulent kinetic energy dissipation $\varepsilon$ in order to determine $\tau_{L}$ for the CBL from Eq. (184). Weil used

$$
\frac{\varepsilon h}{w_{*}^{3}}=1.15 \exp \left(-12.5 \frac{z}{h}\right)-0.2 \exp \left[-50\left(1-\frac{z}{h}\right)\right]+0.3 \text {. }
$$

We now return to Ley and Thomson (1983) for the relation they used for the stable surface layer:

$$
\sigma_{w} / u_{*}=1.5[1-(z / h)]
$$

They got this relation from Caughey et al. (1979) who analyzed stable boundary layer data from the 1973 experiment on the flat, sparsely populated prairie of northwestern Minnesota. Equation (192) is not a scaling relation but merely an empirical fit to the data with the conditions that $\sigma_{w} / u_{*} \rightarrow 1.5$ as $z / h \rightarrow 0$ and $\sigma_{w} / u_{*} \rightarrow 0$ as $z / h \rightarrow 1$. We note that Eq. (192) with $z=0$ is not consistent with Eq. (186); it would be if we changed 1.5 in Eq. (192) to 1.3.

Nieuwstadt (1984a) analyzed stable boundary layer data from Cabauw in the Netherlands as well as the Minnesota data used by Caughey et al. The Cabauw mast is in flat and fairly homogeneous terrain, but the surface roughness is not uniform at all azimuths (which was used to advantage in the paper by Beljaars et al. (1983)). Nieuwstadt's fit to the Cabauw data is

$$
\sigma_{w} / u_{*}=1.4[1-(z / h)]^{3 / 4},
$$

and he noted that the Minnesota data of Caughey et al. tend to lie above that of Cabauw. We see that Eq. (193a) does not reduce to Eq. (186) as $z \rightarrow 0$. However, in a lecture at a conference (Nieuwstadt, 1984b) he gave

$$
\sigma_{w} / u_{*}=1.3[1-(z / h)]^{3 / 4}
$$

"where $1.3 u$. is the value of $\sigma_{w}$ in the surface layer."

Nieuwstadt (1984a) is a key paper worthy of further study because it and a companion paper (Nieuwstadt, 1985) are still, despite some known shortcomings, a definitive frame-work for the idealized stable turbulent boundary layer (Derbyshire, 1990). This is important because the turbulent structure of the stable boundary layer is not as well known as that of the unstable counterpart (Holtslag and Nieuwstadt, 1986; Gryning et al. 1987). Nieuwstadt (1984a) developed a set of seven simplified conservation equations for variances and covariances for the turbulent stable boundary layer. He wrote these equations in terms of eight dimensionless quantities including $\sigma_{w}^{2} / \tau_{u w}$ and $z / \Lambda$ which we will define later. One of these quantities can be independent, leaving a system of seven equations and seven variables. He selected $z / \Lambda$ as the independent variable, and solved the seven equations for the other seven dimensionless variables as functions of $z / \Lambda$. 
Here we pause to define $\tau_{u w}$ and $\Lambda$. The local kinematic shear stress at a given elevation is

$$
\tau_{1 w}=-\langle u w\rangle
$$

where $\langle u w\rangle$ is the local covariance of the longitudinal and vertical velocity fluctuations. The local scaling length at elevation $z$ is

$$
\Lambda=-\tau_{u w}^{3 / 2} \rho C p T / k g H
$$

With reference to Eqs. (128) and (143), $\tau_{u w}^{1 / 2}$ and $\Lambda$ are, respectively, analagous to the friction velocity $u_{*}$ and the Monin-Obukhov scaling length $L$. At $z=0, \tau_{u w}^{1 / 2}=u_{*}$ and $\Lambda=L$.

Nieuwstadt plotted his theoretical solutions, together with the Cabauw data and some of the Minnesota data from Caughey et al. (9), for the seven dependent variables as functions of $z / \Lambda$. In general, he got remarkable agreement between his theoretical model and the data, considering the approximations in the former and the scatter of the latter. Some of his dependent variables varied with $z / \Lambda$ for $z / \Lambda<1$; this corresponds to the local scaling layer shown in Fig. 1 . In all cases, the seven dependent variables approached a constant value as $z / \Lambda \rightarrow \infty$; this corresponds to the $z$-less scaling layer in Fig. 1. It is of special interest to us that Nieuwstadt's theoretical solution as well as the data showed $\sigma_{w} / \tau_{u w}^{1 / 2}$ equal to a constant and independent of $z / \Lambda$ ! His Fig. 2 shows that the fit to the Cabauw data gives approximately

$$
\sigma_{w} / \tau_{u w}^{1 / 2}=1.4
$$

and that the Minnesota data of Caughey et al. give a somewhat higher value (see Eq. (192). Nieuwstadt does not comment on this, but his theoretical solution for $\sigma_{w} / \tau_{u w}^{1 / 2}$ is plotted in his Fig. 2 as a horizontal line at approximately

$$
\sigma_{w} / \tau_{u w}^{1 / 2}=1.3
$$

Nieuwstadt also solved a set of simplified equations for the vertical profiles of the local shear stress $\tau_{u w}$ and the temperature flux, assuming that both the gradient and flux Richardson numbers are equal to 0.2 . He also imposed the boundary conditions that the turbulent fluxes be equal to their surface values at $z=0$ and that they vanish at $z=h$. His result for the momentum flux (shear stress) profile is

$$
\tau_{u w} / u_{*}^{2}=[1-(z / h)]^{3 / 2} .
$$

We get Eqs. (193a-b) from the combinations of Eqs. (196a-b) and (1971). The origins of the exponents 3/4 in Eqs. (193a-b) and 3/2 in Eq. (197) are the mathematical solutions for these profiles, given the selected boundary conditions. This is probably the basis for the following comment in Holtslag and Nieuwstadt (1986): "There is no theoretical justification for [these profiles] and, therefore, they should only be interpreted as convenient approximations and not as generalized turbulence profiles for the stable ABL. As a matter of fact, such profiles would be in contradiction with local scaling, because they depend on surface values of turbulent quantities and they adopt $h$ as a scaling height," However, we have nothing better available for $\sigma_{w} / u_{*}$ in the stable boundary layer so we choose Eq. (193b) which reduces to Eq. (186) as $z \rightarrow 0$. Therefore, with reference to Fig. 1, we have a single height-dependent parameterization for $\sigma_{w} / u_{*}$ for the several stable layers (surface, local scaling, $z$-less scaling) up to but not including the intermittency layer. 
To summarize our selection of parameterizations for $\sigma_{w}$ for use in Langevin diffusion models, we have Eq. (186) from Ley (1982) for the neutral surface layer, Eq. (187) from Panofsky et al. (1977) and Ley and Thomson (1983) for the unstable surface layer, and Eq. (190a) - but with 1.6 changed to 1.69 -from Weil (1990) for the convective boundary layer. We substituted Eq. (193b) from Nieuwstadt (1984b) for the stable surface layer and we discovered that it applies to all of the stable boundary layer up to the intermittency layer. These selected equations have a common solution on the surface where $z=0$, but they do not at the (fuzzy) boundaries between the different scaling regimes shown in Fig. 1-except Eq. (193b) in the stable boundary layer up to the intermittency layer. One of our challenges will be to construct smooth mathematical transitions across these boundaries.

We found several parameterizations for $\varepsilon$ in the applications of the Langevin equation to the convective boundary layer, and we chose Eq. (190b) from Weil (1990) because it is a companion to Eq. (190a). If we use the Langevin equation with $\left(C_{0} \varepsilon\right)^{1 / 2}$ as the coefficient of the stochastic terms, we will need other parameterizations for $\varepsilon$ and we will need to provide transitions across the boundaries from one turbulent scaling regime to another.

\section{Boundary Conditions}

We now concentrate on conditions at two of the boundaries illustrated in Fig. 1: the bottom and the top of the turbulent boundary layer. The former is a "hard" boundary formed by the land or a body of water, and the latter is a relatively "soft" boundary that is more difficult to define. The former can be a "sticky" boundary with surface deposition, and the latter can be "leaky" with transport upward into the entrainment layer or intermittency layer. In diffusion simulations the ground or water surface generally coincides with the bottom of the computational mesh, but the top of the mesh may or may not coincide with the top of the boundary layer. The following discussion is in the context of two-dimensional simulations with vertical $(z)$ and longitudinal $(x)$ coordinates.

In the following papers which we have studied, boundary conditions for the trajectories of marked particles are described, but there is no mention of any problems associated with these conditions. Ley (1982) and Ley and Thomson (1983) assumed perfect reflection at $z=z_{0}$, the surface roughness height, in their simulations of diffusion in the surface layer. Thomson (1984) required perfect reflection at the bottom $(z=0)$ and top $(z=h)$ of turbulent flow in a channel. Thomson (1987) assumed perfect reflection at $z=0$ at the bottom of a free convection layer (see Fig. 1). (We did not include this part of Thomson (1987) in our review of diffusion models for the convective boundary layer because, as noted by Luhar and Britter (1989), that model of Thomson's is valid only for the lower part of the CBL where the turbulence skewness is very small.) Baerentsen and Berkowicz (1984) and deBaas et al. (1986) assumed perfect reflection at both the bottom $(z=0)$ and top $(z=h)$ of the CBL.

We studied Boughton et al. (1987) in connection with the Markov or diffusion equation limit of the Langevin equation. Their diffusion model is based on random displacement increments instead of random velocity increments. They commented, "Previous stochastic models of atmospheric diffusion have had considerable difficulty correctly simulating the boundary case for other than perfect reflection ...," but they did not identify any specific cases. They used a transition probability density in their boundary condition for particle deposition on the ground. This was a principal 
contribution of their paper, and they recently published a note (1989) to clarify some questions that had been raised.

However, perfectly-reflecting boundary conditions can cause problems. Two examples were mentioned by van Dop et al. (1985). First, numerical accuracy requires that $\Delta t \ll \tau_{L}$ where $\Delta t$ is the incremental time step and $\tau_{L}$ is the Lagrangian decorrelation time scale. This condition may be violated near a boundary if $\tau_{L}$ is a function of position (see, for example, Eqs. (136)-(137) for Ley's (1982) simulation of diffusion in the neutral surface layer). van Dop et al. also observed that the vanishing of $\tau_{L}$ near a boundary (as in Ley) raises the question whether or not particles can reach such a boundary. Under some conditions (e.g., Eqs. (136)-(137)), the answer is "no." They cautioned that care should therefore be used in predicting surface concentrations and commented that it is likely that finite-difference solutions of surface concentrations are dependent on grid size.

Sawford and Guest (1987) were specific in discussing problems associated with the perfectlyreflecting boundaries at the top and bottom in deBaas et al. (1986). They observed that the model of deBaas et al. did not preserve the well-mixed condition for downwind, but gave concentrations about 0.7 times the homogeneous value in two thin layers along the top and bottom boundaries. They noted that the reflection condition requires that the skewness of the turbulence be zero at the boundary and that the model of deBaas et al. does not meet this condition. They demonstrated that a modified deBaas et al. model with zero skewness gave much better results at the top reflecting boundary (and worse results at the bottom because of the turbulence inhomogeneity near that boundary). Their model gave better results in inhomogeneous turbulence, and they used parameterizations for the vertical velocity variance and skewness that vanished at the reflecting

boundaries. Consequently these boundaries could not be reached so they presented near-surface concentrations, not surface concentrations, as results. They also admitted that the perfect reflection condition at the top of the CBL is not strictly correct.

Luhar and Britter (1989) used the same boundary conditions and the same parameterizations for velocity variance and skewness as Sawford and Guest. Their only cumment about problems at the boundaries was that the use of large values of $\Delta t$ did not give the proper behavior of particles near boundaries.

Weil's (1990) principal contribution to the boundary condition problem is given for homogeneous skewed turbulence in Sec.3.b and Appendix B of his paper. He modified the reflection boundary condition to account for the asymmetry of the probability distribution function (PDF) of the vertical velocity fluctuations in the CBL.

We close this section with two observations. First, there are several dimensions to the croblem of designing appropriate boundary conditions for the Langevin model for turbulent diffusion. Second, none of the references found contain a treatment of the problem in much depth or detail.

\section{Experimental Data Sets and Test Problems}

We next review experimental data sets and test problems that have been used for evaluating and demonstrating the several versions of the Langevin model for turbulent diffusion. We will consider test problems first and then data sets from experiments (field, laboratory, and numerical). We will also note data sets that have not been used with Langevin models but could be. 
Two different kinds of test problems with known solutions have been used. The first kind involves idealized problems with analytic solutions; the second is based on the second law of thermodynamics.

If the eddy-diffusivity or K-theory of turbulent diffusion is applicable, analytic solutions of the diffusion equation can legitimately be compared to Markov or diffusion limit solutions of the Langevin equation. This approach was used by Boughton et al. (1987) who compared results from their random-displacement model with analytic solutions obtained by Ermak (1977) and Rounds (1955). This approach was also used by Wilson et al. (1983) in their first comparison of the different. "drift corrections" in Eqs. (30b) and (31b). This comparison did not give a clear indication that one correction was better than the other for use in inhomogeneous turbulence, but a second comparison (which we will now describe) clearly indicated the superiority of Eq. (30b) for strongly inhomogeneous turbulence.

One of the consequences of the second law of thermodynamics is that a population of "marked" particles released into inhomogeneous turbulence will eventually become "well-mixed" and will stay that way. This principle makes it possible to design a problem with complex initial and boundary conditions whose long-term solution is known from physics, not necessarily from mathematics. This technique was used by Wilson et al. (1983) in their second test of Eq. (30b) vs. Eq. (31b). It was also used by Legg and Raupach (1982) to test Eq. (31b) and by Thomson (1984) for several comparisons including Eq. (30b) vs. Eq. (31b). We have seen in preceding sections that Thomson $(1984,1987)$ put Eq. (30b) on a firm theoretical basis thereby eliminating one need for test problems. Thomson's principles were used by Luhar and Britter (1989) and Weil (1990) in developing their common form of the Langevin model for inhomogeneous non-Gaussian turbulence from Gaussian forcing in the CBL.

We found only four sources o development and application of the chemical traces, two over flat terrain and two over complex terrain. Ley (1982) and Ley and Thomson (1983) compared data from the Kansas prairie and Porton Down in England with their numerical results for diffusion in the surface layer. These data are plotted as points in figures in these papers. Barad (1985) is cited as the source for the Kansas data; this reference may not be readily available. T, e data from Porton Down was published by Thompson (1971). Thomson (1986) presented both diffusion data and simulation results for experiments in a valley in South Wales. Yamada and Bunker (1988) compared their model results with data from the experiment at Brush Creek in the Colorado mountains. Again, these data are plotted as points in figures in these papers.

The review by Gryning et al. (1987) does not include use of the Langevin model but does cite a number of diffusion measurements in the field, including the above Kansas data. They did not cite Barad (1958) but several other papers in which Kansas data were plotted and used; the last reference for our purposes appears to be Gryning et al. (1983). The Kansas data are presented in scaled form in the papers cited by Gryning et al. (1987) so they may have to be rescaled for our purposes. Scaled data from otr 2 r experiments mentioned by Gryning et al. (1987) include Irwin (1983), Gryning anc: Lyck (1984), and Doran and Horst (1985). Finally, we at LLNL have participated in several field programs with which we are quite familiar. 
To return to data used with Langevin models, Sawford and Guest (1988) used data for temperature diffusion downwind of a heated wire in a neutral surface layer in a wind tunnel (Legg, 1983).

The water tank simulations of a convective boundary layer that were conducted by Willis and Deardorff $(1974,1976,1978,1981)$ and Deadorf and Willis (1985) are the principal source of experimental data for comparison with Langevin model results. Turbulent convection was initiated and maintained by heating the bottom of the tank. Neutrally-buoyant oil droplets were injected from a line source at various heights. The diffusion of these droplets were recorded by photographs taken every few seconds. One or more of these papers were cited and used for model comparisons by Baerentsen and Berkowicz (1984), van Dop et al. (1985), deBaas et al. (1986), Sawford and Guest (1987), Luhar and Britter (1989), and Weil (1990).

The authors of the above CBL papers also cited numerical simulations of the convective boundary layer by Lamb $(1978,1982)$. Lamb compared his results with some of the above water tank data of Willis and Deardorff. Lamb's Lagrangian model is based on particle velocities that are the sum of a deterministic component and a stochastic component. The deterministic component is taken from Large Eddy Simulations (LES) and the stochastic component represents the sub-grid fluctuations. The particle velocity equation in Lamb (1978) is analogous to the Langevin equation but that in Lamb (1982) is not because there is some persistence of "memory" for the stochastic component; see Lamb (1981) for his description of various levels of the Markov process. Lamb's contributions were highly valued at the time they were published, but it appears that his line of modeling has not been continued.

\section{Summary}

The following applies to turbulent diffusion in one dimension (the vertical) unless otherwise stated. Some specific problems associated with extension of the Langevin model to two and three dimensions are identified.

The structure of turbulence in the idealized, horizontally-homogeneous atmospheric boundary layer can be described in terms of various scaling parameters for different turbulence regimes (see Fig. 1 and the short review by Holtslag and Nieuwstadt (1986). Monin-Obukhov scaling has long been established for the surface layer (unstable, neutral, stable) but there is still uncertainty about the "best" empirical fits of data to the scaling functions (I. Iglom, 1977). The scaling for the unstable convective boundary layer has been verified both by experiments and numerical simvlations (e.g., the companion papers by Willis and Deardorff (1978) and Lamb (1978). There is no generally accepted scaling for the stable boundary layer, but Nieuwstadt's theory is the most promising (Nieuwstadt, 1984a, 1985; Derbyshire, 1990). There are, at present, no scaling relations for the near-neutral upper layer, the entrainment layer, and the intermittency layer. There are relatively few data and publications concerning scaling of horizontal turbulence, vith the possible exception of the unstable surface layer (e.g., Panofsky, et al. 1977).

The capability of the Langevin model to simulate turbulent scalar diffusion in the surface layer over flat terrain was proven by the mid-1980s (Ley, 1982; Ley and Thomson, 1983), but there is need for further development for application to the surface layer over complex terrain (Thomson, 1986; Yamada and Bunker, 1988). The greatest advances during recent years have been made in applying the Langevin model to diffusion in the convective boundary layer, beginning with 
Baerentsen and Berkowicz (1984) and continuing through Weil (1990). The relative success (or failure) of these efforts has been a complex function of (1) the form of the Langevin equation, (2) the turbulence parameterizations, (3) the boundary conditions, and (4) the experimental data sets and test problems used for verification. In addition, the accuracy of the mean flow field is important in complex terrain.

The ultimate form of the Langevin to date, the combination of Eqs. (168)-(169), was derived independently by Luhar and Britter (1989) and Sawford (Weil, 1990) for the convective boundary layer. It is based on criteria by Thomson (1987), including the necessity for Gaussian forcing (the input) and the method of Baerentsen and Berkowicz (1984) for specifying the first three moments of non-Gaussian turbulence (the output) in terms of the sum of two Gaussian distributions. For Gaussian inhomogeneous turbulence, Eqs. (168)-(169) reduce to Eq. (30b) which includes the drift correction of Wilson et al. (1981) as modified by Wilson et al. (1983). This drift correction was put on a rigorous basis by Thomson (1984) and Sawford (1986). Equation (30b) should be used for the surface layer instead of that used by Ley and Thomson (1983). For the constant-stress region in the neutral surface layer, Eq. (30b) reduces to Eq. (19) which was used by Ley (1982).

There may be cases of inhomogeneous turbulence in addition to the convective boundary layer, for which Eqs. (168)-(169) should be used instead of Eq. (30b). Pope (1987) was critical of both Thomson (1984) and Sawford (1986) for their use of Gaussian inhomogenous turbulence as a test case (e.g., with Eq. (30b)). He stated that such a specification is physically incorrect because inhomogenous turbulence is not Gaussian. van Dop et al. (1985) also commented that inhomogeneous turbulence is generally observed to be non-Gaussian.

The above applies only to diffusion in one dimension. As noted on p. 543 of Thomson (1987) and demonstrated by Sawford and Guest (1988), there are presently no criteria for obtaining a unique form of the Langevin equation for turbulent diffusion in more than one dimension.

Given the proper form of the Langevin equation, the next requirement is for values of the input parameters. These are, as a minimum, the local decorrelation time scale $\tau_{L}$ and the velocity scale $\sigma_{w}$ where $\sigma_{w}{ }^{2}$ is the variance of the velocity fluctuations. These inputs are function of the scaling parameters for the different turbulence regimes of the atmospheric boundary layer.

Assuming a parametric relation for $\sigma_{w}$ is available, it is relatively simple to determine $\tau_{L}$ for the surface layer because Monin-Obukhov scaling is applicable and the Markov or diffusion equation limit of the Langevin equation is valid (see Eq. (170)). The trend during the development of the Langevin equation for the convective boundary layer was toward substituting $C_{0} \varepsilon$ for $2 \sigma_{w}{ }^{2} / \tau_{L}$ as the coefficient of the stochastic term (see Eq. (176)) where $\varepsilon$ is the rate of dissipation of turbulent kinetic energy and $C_{0}$ is the universal constant for the Lagrangian structure function. This substitution can be used for all turbulence regimes in the atmospheric boundary layer, providing that a parametric relation for $\varepsilon$ is available and the value of $C_{0}$ is known. There has been, and still is, uncertainty about the value of $C_{0}$. Estimates have ranged from about 2 to 10 ; we have calculated $C_{0}=5.7$ (see Eq. (185)).

We have a set of equations for $\sigma_{w} / u_{*}$ where $u_{*}$ is the surface friction velocity: Eq. (136) for the constant-stress region in the neutral surface layer, Eq. (187) for the unstable surface layer, Eq. (190a) for the convective boundary layer, and Eq. (193b) for the stable boundary layer. These equations have the common solution of $\sigma_{w}=1.3 u_{*}$ on the ground surface $(z=0)$, but their solutions are not continuous across the transitions from one turbulence regime to another (see 
Fig. 1). Furthermore, Eq. (193b) for the stable boundary layer is not a true scaling relation but merely a convenient approximation. There is a great need for additional development in this area.

We have empirical parametric relations for the third moment of the velocity fluctuations $\left\langle w^{3}\right\rangle$ (Eq. (190b)) and the dissipation rate $\varepsilon$ (Eq. (191)) for only the convective boundary layer.

We clearly have an imperfect and incomplete set of parametric equations for the inputs to the Langevin equation. Furthermore, our algorithms for calculating $\tau_{L}, \sigma_{w}, \varepsilon$, etc. must include the means to determine the values of scaling parameters like the friction velocity $u_{*}$, the convective velocity scale $w_{*}$, the Monin-Obukhov scaling length $L$, the boundary layer height $h$, etc.

Assuming that the appropriate form of the Langevin equation and parametric relations for its inputs are on hand, our modeling system is incomplete without boundary conditions. The treatment of boundary conditions in the literature is uneven. There is evidence that incorrect boundary conditions can cause serious errors in the results, that defining correct boundary conditions for imperfectly reflecting boundaries and non-Gaussian probability distributions are not trivial tasks, and that there are complex interactions among the three components of the system (Langevin equation, parameterizations for the inputs, and boundary conditions).

Finally, there are experimental data sets and test problems for use in verifying our diffusion model. There are a few test problems with known analytic solutions for specific cases. There is one general test problem based on physics: the dispersing material will eventually become well-mixed and will stay that way. There are some experimental results (field, laboratory, numerical) for some, but not all, meteorological conditions of interest.

\section{References}

Anand, M.S., and S.B. Pope, 1985: Diffusion behind a line source in grid turbulence. Turbulent Shear Flows 4, edited by L.J.S. Bradbury, F. Durst, B.E. Launder, F.W. Schmidt, and J.H. Whitelaw. Springer-Verlag, Berlin, pp. 46-61.

Baerentsen, J.H., and R. Berkowicz, 1984: Monte Carlo simulation of plume diffusion in the convective boundary layer. Atmiss. Environ., 18, 701-712.

Barad, M.L., 1958: Project Prairie Grass, A Field Program in Diffusion, Vol. 1. Geophysics Research Paper, 59, Air Force Cambridge Research Center AFCRC-TR-58-235.

Beljaars, A.C.M., P. Schotames, and F.T.M. Nieuwstadt, 1983: Surface layer similarity under nonuniform fetch conditions. J. Climate Appl. Meteorol., 22, 1800-1820.

Boughton, B.A., J.M. Delaurentis, and W.E. Dunn, 1987: A stochastic model of particle diffusion in thr atmosphere. Boundary-Layer Meteorol., 40, 147-163. 1989: 48, 443-444.

Calder, K.L., 1966: Concerning the similarity theory of A.S. Monin and A.M. Obukhov for the turbulent struture of the thermally stratified surface layer of the atmosphere. Quart. J.R. Met. Soc., 92, 141-146.

Caughey, S.J., and S.G. Palmer, 1979: Some aspects of turbulence scructure through the depth of the convective boundary layer. Quart. J.R. Met. Soc., 105, 811-827. 
deBaas, A.F., H. van Dop, and F.T.M. Nieuwstadt, 1986: An application of the Langevin equation for inhomogeneous conditions to diffusion in the convective boundary layer. Quart. J.R. Met. Soc., 112, 165-180.

Deardorff, J.W., and G.E. Willis, 1985: Further results from a laboratory model of the convective planetary boundary layer. Boundary-Layer Meteorol., 32, 205-236.

Derbyshire, S.H., 1990: Nieuwstadt's stable boundary layer revisited. Quart. J.R. Met. Soc., 116, 127-158.

Doran, J.C., and T.W. Horst, 1985: An evaluation of Gaussian plume-depletion models with dualtracer field measurements. Atmos. Environ., 19, 939-951.

Ermak, D.L., 1977: An analytical model for air pollutant transport and deposition from a point source. Atmos. Environ., 11, 231-237.

Gryning, S.E., A.P. van Ulden, and S. Lassen, 1983: Diffusion from a continuous ground-level source investigated by a K-model. Quart. J.R. Met. Soc., 109, 355-364.

Gryning, S.E., and E. Lyck, 1984: Atmospheric diffusion from elevated sources in an urban area: Comparison between tracer experiments and model calculations. J. Climate Appl. Meteorol., 23, 651-660.

Gryning, S.E., A.A.M. Hotslag, J.S. Irwin, and B. Sivertsen, 1987: Applied diffusion modeling based on meteorological scaling parameters. Atmos. Environ., 21, 79-89.

Hanna, S.R., 1981: Lagrangian and Eulerian time-scale relations in the daytime boundary layer. J. Appl. Meteorol., 20, 242-249.

Holtslag, A.A.M., and F.T.M. Nieuwstadt, 1986: Scaling the atmospheric boundary layer. BoundaryLayer Meteorol., 36, 201-209.

Irwin, J.S., 1983: Estimating plume diffusion-A comparison of several sigma schemes. J. Climate Appl. Meteorol., 22, 92-114.

Lamb, R.G., 1978: A numerical simulation of diffusion from an elevated point source in the convective planetary boundary layer. Atmos. Environ., 12, 1297-1304.

Lamb, R.G., 1981: A scheme for simulating particle pair motions in turbulent fluid. J. Comp. Physics, 39, 329-346.

Lamb, R.G., 1982: Diffusion in the convective boundary layer. Atmospheric Turbulence and Air Pollution Modeling, edited by F.T.M. Nieuwstadt and H. van Dop. D. Reidel, Dortrecht.

Legg, B.J., 1983: Turbulent diffusion from an elevated lines sources: Markov chain simulations of concentration and flux profiles. Quart. J.R. Met. Soc., 109, 645-660.

Legg, B.J., and M.R. Raupach, 1982: Markov-chain simulation of particle diffusion in inhomogeneous flows: The mean drift velocity induced by a gradient in Eulerian velocity variance. Boundary-Layer Meteorol., 24, 3-13.

Ley, A.J., 1982: A random walk simulation of two-dimensional turbulent diffusion in the neutral surface layer. Atmos. Environ., 16, 2799-2808.

Ley, A.J., and D.J. Thomson, 1983: A random walk model of diffusion in the diabatic surface layer. Quart. J.R. Met. Soc., 109, 867-880. 
Luhar, A.K., and R.E. Britter, 1989: A random walk model for diffusion in inhomogeneous turbulence in a convective boundary layer. Atmos. Environ., 23, 1911-1924.

Monin, A.S., and A.M. Yaglom, 1971, 1975: Statistical Fluid Mechanics: Mechanics of Turbulence, Vol. 1 and 2. MIT Press, Cambridge.

Nieuwstadt, F.T.M., 1984a: The turbulent structure of the stable, nocturnal boundary layer. $J$. Atmos. Sci., 41, 2202-2216.

Nieuwstadt, F.T.M., 1984b: Some aspects of the turbulent stable boundary layer. Boundary-Layer Meteorol., 30, 31-55.

Nieuwstadt, F.T.M., 1985: A model for the stationary, stable boundary layer. Turbulence and diffusion in stable environments, edited by J.C.R. Hunt. Oxford University Press, pp. 149-179.

Panofsky, H.A., H. Tennekes, D.H. Lenschow, and J.C. Wyngaard, 1977: The characteristics of turbulent velocity components in the surface layer under convective conditions. BoundaryLayer Meteorol., 14, 235-246.

Pope, S.B., 1983: A Lagrangian two-time probability density function equation for inhomogeneous turbulent flows. Phys. Fluids, 26, 3448-3450.

Pope, S.B., 1987: Consistency conditions for random-walk models of turbulent diffusion. Phys. Fluids, 30, 2374-2379.

Pope, S.B., and Y.L. Chen, 1990: The velocity-dissipation probability density function model for turbulent flows. Phys. Fluids, 42, 1437-1449.

Rodean, H.C., 1991: The universal constant for the Lagrangian structure function. Phys. Fluids.. A3, 1479-1480.

Rounds, W., 1955: Solutions of the two-dimensional diffusion equation. Trans. Amer. Geophys. Union, 36, 395-405.

Sawford, B.L., 1985: Lagrangian statistical simulation of concentration mean and fluctuation fields. J. Climate Appl. Meteorol., 24, 1152-1166.

Sawford B.L., 1986: Generalized random forcing in random-walk turbulent diffusion models. Phys. Fluids, 29, 3582-3585.

Sawford, B.L., and F.M. Guest, 1987: Lagrangian stochastic analysis of flux-gradient relationships in the convective boundary layer. J. Atmos. Sci., 44, 1152-1165.

Sawford, B.L., and F.M. Guest, 1988: Uniqueness and universality of Lagrangian stochastic models of turbulent diffusion. Proceedings of the 8th Symp. on Turbulence and Diffusion. Amer. Meteorol. Soc., Boston, 96-99.

Thomson, D.J., 1984: Random walk modeling in diffusion in inhomogeneous turbulence. Quart. J.R. Met. Soc., 110, 1107-1120.

Thomson, D.J., 1986: A random walk model of diffusion in turbulent flows and its application to diffusion in a valley. Quart. J.R. Met. Soc., 112, 511-530.

Thomson, D.J., 1987: Criteria for the selection of stochastic models of particle trajectories in turbulent flows. J. Fluid Mech., 180, 529-556. 
Thompson, N., 1965: Short range vertical diffusion in stable conditions. Quart. J.R. Mct. Soc., 91, 175-183.

Townsend, A.A., 1976: The structure of turbulent shear flow. Cambridge University Press.

van Dop, H., F.T.M. Nieuwstadt, and J.C.R. Hunt, 1985: Random walk models for particle displacements in inhomogeneous unsteady turbulent flows. Phys. Fluids, 28, 1639-1653.

van Ulden, A.P., and A.A.M. Holtslag, 1985: Estimation of atmospheric boundary layer parameters for diffusion applications. J. Climate Appl. Meteorol., 24, 1196-1207.

Weil, J.C., 1990: A diagnosis of the asymmetry in top-down and botton-up diffusion using a Lagrangian stochastic model. J. Atmos. Sci., 47, 501-515.

Willis, G.E., and J.W. Deardorff, 1974: A laboratory model of the unstable planetary boundary layer. J. Atmos. Sci., 31, 1297-1307.

Willis, G.E., and J.W. Deardorff, 1976: A laboratory model of diffusion into the convective planetary boundary layer. Quart. J.R. Met. Soc., 102, 427-445.

Willis, G.E., and J.W. Deardorff, 1978: A laboratory study of diffusion from an elevated source within a modeled convective planetary boundary layer. Atmos. Environ., 12, 1305-1311.

Willis, G.E., and J.W. Deardorff, 1981: A laboratory study of diffusion from a source in the middle of the convective mixed layer. Atmos. Environ., 15, 109-117.

Wilson, J.D., B.J. Legg, and D.J. Thomson, 1983: Calculation of particle trajectories in presence of a gradient in turbulent-velocity variance. Boundary-Layer Meteorol., 27, 163-169.

Wilson, J.D., G.W. Thurtell, and G.E. Kidd, 1981: Numerical simulation of particle trajectories in inhomogeneous turbulence, II: Systems with variable turbulence velocity scale. BoundaryLayer Meteorol., 21, 423-441.

Yaglom, A.M., 1977: Comments on wind and temperature flux-profile relationships. BoundaryLayer Meteorol., 11, 89-102.

Yamada, T., and S. Bunker, 1988: Development of a neutral grid, second moment turbulence closure model and application to the 1982 ASCOT Brush Creek simulation. J. Appl. Meteorol., 27, 562-578.

Langevin Notes, Part II.tex 


\title{
Part III: Toward Implementation of Stochastic Models for Diffusion
}

\begin{abstract}
Two Lagrangian stochastic models for turbulent diffusion, Langevin equation and random displacment, are described and compared. The comparison is extended to include the ranges of time $t$ and value of the time increment $\Delta t$ that are appropriate for each model. Assuming stationary, horizontally homogeneous, vertically inhomogeneous, Gaussian turbulence, the stochastic differential equations for both models of diffusion in one, two, and three dimensions are presented. Parameterizations of the turbulence statistics used as model inputs are proposed for the full range of dimensionless height and stability of the atmospheric boundary layer. The Ito Calculus for integrating these stochastic differential equations is described and applied, as an illustration, to the Langevin model for vertical diffusion.
\end{abstract}

\section{Introduction}

This part of the manuscript consists of materials that were gathered or developed in preparation for implementation of two stochastic models, the Langevin model and the random dispacement model, in the ADPIC dispersion model (Lange, 1978). Some of the following is based on independent work and some is the result of joint work with my colleagues Don Ermak, Vladimir Gavrilov, Rolf Lange, and John Nasstrom. The emphasis in Part II of this manuscript is on the Langevin model, but the random displacement model and its relation to the Langevin model are described in the Section, "The Markov or Diffusion Equation Limit." The decision to implement the random displacement model as well as the Langevin model in ADPIC is a result of a cooperative research effort between the Lawrence Livermore National Laboratory and the Institute of Experimental Meteorology (see Part I).

\section{The Langevin and Random Displacement Models for Diffusion}

For the purposes of illustration in this Section, we assume a Gaussian distribution of random velocity fluctuations in stationary turbulent flow that is horizontally homogeneous and vertically inhomogeneous. We consider one-dimensional turbulent diffusion with zero mean flow along the vertical coordinate. 


\section{The Relation between the Langevin and Random Displacement Models}

The Langevin model consists of a pair of stochastic differential equations for $w(z, t)$ and $z(t)$ that describe the trajectories of neutrally-buoyant "marked" particles:

$$
d w=\left[-\frac{w}{\tau}+\frac{1}{2}\left(1+\frac{w^{2}}{\sigma_{w}^{2}}\right) \frac{\partial \sigma_{w}^{2}}{\Gamma z}\right] d t+\left(\frac{2 \sigma_{w}^{2}}{\tau}\right)^{\frac{1}{2}} d W(t)
$$

and

$$
d z=w d t
$$

Here $w$ is the vertical Lagrangian velocity $\sigma_{w}^{2}$ is the Eulerian variance of $w, \tau$ is a local velocity decorrelation time scale that has an exact definition only in homogeneous turbulence, $z$ is the vertical coordinate, $t$ is time, and $d W(t)$ is the incremental Wiener process with zero mean and variance $d t$. The $d t$ term in Eq. (1a) is deterministic and the $d W(t)$ term is stochastic. The deterministic term has two components, the first is a "fading memory" term associated with particle velocity decorrelation, the second component is a "drift correction" which accounts for the vertical inhomogeneity of the turbulence. The stochastic term is a model of the effect of the random pressure fluctuations on the particle velocity,

Equations (1a-b) were first proposed by Wilson et al. (1983) and were given a rigorous foundation by Thomson (1984), Sawford (1986), and Thomson (1987). These equations or their equivalent are discussed at more length in Part I (The Langevin Model) and Pari II (Drift Corrections for Inhomogeneous Turbulence).

Under the condition that the time scale $\tau \rightarrow 0$ (or $t / \tau \rightarrow \infty$ ), the Langevin model Eqs. (1a-b) can be transformed into a stochastic differential equation for $z(t)$, the random displacement model. This transformation was given by Durbin (1984) and, in more detail, by de Baas and Troen (1989). The procedure of de Baas and Troen is given in Part II (The Markov or Diffusion Equation I.imit). The transformation includes a rescaling of the turbulence with $\tau \rightarrow 0$ and $\sigma_{w} \rightarrow \infty$ such that the quantity $\sigma_{w}^{2} \tau=K$ remains constant. The result is

$$
d z=\frac{\partial}{\partial z}\left(\sigma_{w}^{2} \tau\right) d t+\left(2 \sigma_{w}^{2} \tau\right)^{\frac{1}{2}} d W(t)
$$

which is the Lagrangian equivalent of the Eulerian eddy diffusivity model. Here $z(t)$ is the only variable and the deterministic term consists only of a "drift correction" for inhomogeneous turbulence.

\section{Ranges of Time $t$ and Time Increment $\Delta t$ for the Two Models}

The above stochastic differential equations, Eqs. (1a-b) and (2), are integrated according to the rules of the Ito Calculus (Arnold, 1974, pp 60 and 71; van Kampen, 1981, p 244; Gardiner, 1983 , pp 84 and 88-92). These equations have valid mathematical solutions for all values of time $t \geq 0$, but these solutions are not valid models of turbulent diffusion for all positive values of time. The reason for this discrepancy between mathematics and physics is to be found in the difference between the properties of the stochastic terms involving the incremental Wiener process $d W(t)$ and the properties of the turbulence field. 
The stochastic terms of Eqs. (1a) and (2) containing the incremental Wiener process $d W(t)$ result in outputs $d w_{s}$ and $d z_{s}$, respectively, that are continuous but are not differentiable (the subscript $s$ refers to the stochastic portion of the solution) (see Arnold, pp 35, 45, and 134-136; van Kampen, pp 84 and 218; Gardiner, pp 66-70). Consequently the solutions of Eqs. (1a) and (2) imply infinite particle accelerations and velocities, respectively. In finite-difference calculations, the outputs of these equations may be visualized as the sum of (1) the change in $w$ (or $z$ ) due to the deterministic term acting over the $\Delta t$ and (2) the change in $w$ (or $z$ ) due to the instantaneous action of the stochastic term (see Fig. 4.2 on p 93 of Gardiner).

The ranges of validity of the Langevin and random displacement models are related to Kolmogorov's inertial subrange,

$$
\tau \gg t \gg \tau_{k}
$$

for high Reynolds number, three dimensional turbulence where $\tau_{k}$ is the Kolmogorov time scale (Lin and Reid, 1963, pp 515-516; Monin and Yaglom, 1975, pp 345-377 and 547-551; Sawford, 1984; Thomson, 1984, 1987; Boughton et al., 1987). The Langevin Model, Eqs. (1a-b) is assumed to be valid for

$$
t \gg \tau_{k}
$$

because $\tau_{k}$ is the time scale over which particle accelerations are correlated. The random displacement model is assumed to be valid for

$$
t \gg \tau
$$

because $\tau$ is the time scale over which particle velocities are correlated.

The time increment $\Delta t$ which is physically appropriate for numerical solutions of the Langevin model is related to the Lagrangian structure function $D(\Delta t)$ (Monin and Yaglom, 1975, pp 83 and 359; Thomson, 1987) where

$$
D(\Delta t)=\left\langle(\Delta w)^{2}\right\rangle=C_{0} \varepsilon \Delta t
$$

with

$$
\tau \gg \Delta t \gg \tau_{k}
$$

Here $C_{0}$ is a universal constant and $\varepsilon$ is the mean rate of dissipation of turbulence kinetic energy. However, the properties of the incremental Wiener process $d W(t)$ are such that, in effect, $\tau_{k}=0$ in Eq. (1a) so $\Delta t$ is constrained by only the upper limit of Eq. (6b). Similarly, $\tau=0$, in effect, in Eq. (2) for the random displacement model so $\Delta t$ is not constrained by a finite lower limit in numerical calculations (we remember that $\tau \rightarrow 0$ in the transformation of Eqs. (1a-b) into Eq. (2)). The upper limit for $\Delta t$ for the random displacement model is a presently ill-defined function of the scale of inhomogeneity of the turbulent flow field.

\section{Langevin Models for Diffusion in Gaussian Turbulence}

Our objective is to implement a Langevin model for turbulent diffusion in three dimensions in ADPIC. Mathematically rigorous Langevin models for three dimension are available for Gaussian turbulence but not for the non-Gaussian case. Therefore we consider only Gaussian turbulence in this section. We begin by considering the model for three dimensions. We continue by reducing it to two dimensions (lateral and vertical) and then to one dimension (vertical). In our implementation, w. will begin with the one dimensional model and then proceed to the two and three dimensional cases. This is in the interest of simplying the debugging and testing procedures. 


\section{"Simplest" Model for Diffusion in Three Dimensions}

The following is based on the work of Thomson (1987) who stated that it is possible to obtain a unique solution to the Fokker-Planck equation (and therefore a unique form for the Langevin equation) for turbulent diffusion in one dimensions but not in two or three. He presented what he called "the simplest solution" for three dimensions. Sawford and Guest (1988) used this model and another three dimensional model developed by M.S. Borgas. We will use the "simplest" model by Thomson.

We start with the Langevin type, stochastic differential equations

$$
\begin{gathered}
d x_{i}=u_{i} d t \\
d u_{i}=a_{i}(\mathbf{x}, \mathbf{u}, t) d t+b_{i j}(\mathbf{x}, \mathbf{u}, t) d W_{j}(t)
\end{gathered}
$$

where $x_{i}$ is displacment; $u_{i}$ is velocity; $\mathbf{a}$ and $\mathbf{b}$ are functions of $\mathbf{x}, \mathbf{u}$, and $\mathrm{t}$; and the $d W_{j}(t)$ are the increments of a vector-valued Wiener piocess with independent increments. The mean of $d W_{j}$ is zero and its variance is $d t$; the increments of $d W_{j}$ at different times, or at the same time with $i \neq j$ are independent. Unlike the solution for $a_{i}$, that for $b_{i j}$ is simple:

$$
b_{i j}=\delta_{i, j}\left(C_{0} \varepsilon\right)^{1 / 2}
$$

where $\delta_{i j}$ is the Kronecker delta with $\delta_{i j}=1$ if $i=j$ and $\delta_{i j}=0$ if $i \neq j, C_{0}$ is the universal constant associated with the Lagrangian structure function, and $\varepsilon$ is the rate of dissipation of turbulence kinetic energy. For each value of $i$ (where $i=1,2,3$ corresponding to the coordinates $x, y, z$ respectively), the right side of Eq. (8) has one component.

Part of the solution for $a_{i}$ is

$$
a_{i}=-\delta_{i j} \frac{C_{0} \varepsilon}{2} \Gamma_{j k}\left(u_{k}-\overline{u_{k}}\right)+\frac{\phi_{i}}{g_{a}}=-\frac{C_{0} \varepsilon}{2} \Gamma_{i k}\left(u_{k}-\overline{u_{k}}\right)+\frac{\phi_{i}}{g_{a}} .
$$

Here $\bar{u}_{k}$ is a mean Eulerian velocity and $\Gamma_{i k}$ is the inverse of the kinematic Reynolds stress tensor. assuming incompressible flow. Thomson assumed variable-density flow in most of his development. but we will follow Sawford and Guest (1988) in assuming constant-density flow. For each value of $i$, the first term on the right side of Eq. (9) has three components.

Thomson (1987) presented his Eq. (32) as the simplest of the possible forms for $\phi_{i} / g_{a}$ :

$$
\begin{gathered}
\frac{\phi_{i}}{g_{a}}=\frac{\partial \overline{u_{i}}}{\partial t}+\bar{u}_{l} \frac{\partial \overline{u_{i}}}{\partial x_{l}}+\frac{\partial \overline{u_{i}}}{\partial x_{j}}\left(u_{j}-\overline{u_{j}}\right)+\frac{1}{2} \frac{\partial \tau_{i l}}{\partial x_{l}}+\frac{\Gamma_{l j}}{2} \frac{\partial \tau_{i l}}{\partial t}\left(u_{j}-\overline{u_{j}}\right) \\
+\frac{\Gamma_{l j}}{2}\left(\overline{u_{m}} \frac{\partial \tau_{i l}}{\partial x_{m}}\right)\left(u_{j}-\overline{u_{j}}\right)+\frac{\Gamma_{l j}}{2} \frac{\partial \tau_{i l}}{\partial x_{k}}\left(u_{j}-\overline{u_{j}}\right)\left(u_{k}-\overline{u_{k}}\right) .
\end{gathered}
$$

The first term on the right has one index, the next three have two indices, the fifth term has three, and the last two have four. This means that, in the general case, $\phi_{i} / g_{a}$ for each $i$ has a total of 73 components. In other words, with $i=i$ and $j, k, l, m=1,2,3$; the right side of Eq. (10) has a total of 73 terms in its expanded form (we retain the velocity differences $u_{j}-\overline{u_{j}}$ and $u_{k}-\overline{u_{k}}$. It is clear that some simplification is essential if we are to use Eq. (10) in practical applications. 
We ass' 'me stationary turbulent flow $(\partial / \partial t=0)$ that is horizontally homogeneous

$$
\left(\partial / \partial x_{1}=\partial / \partial x_{2}=0 \text { or } \partial / \partial x=\partial / \partial y=0\right)
$$

with zero mean lateral and vertical flow $\left(\bar{u}_{2}=\bar{u}_{3}=0\right.$ or $\left.\bar{v}=\bar{w}=0\right)$. The result is turbulent flow that can be vertically inhomogeneous $\left(\partial / \partial x_{3} \neq 0\right.$ or $\left.\partial / \partial z \neq 0\right)$ and is aligned with the longitudinal $x_{1}$ or $\left.x\right)$ coordinate $\left(\bar{u}_{1} \neq 0\right.$ or $\left.\bar{u} \neq 0\right)$. Under these conditions, it can be shown (see e.g., Calder, $1966)$ that the Reynolds stress tensor reduces to

$$
\tau_{i j}=\left|\begin{array}{ccc}
\tau_{11} & 0 & \tau_{13} \\
0 & \tau_{22} & 0 \\
\tau_{31} & 0 & \tau_{33}
\end{array}\right|
$$

with

$$
\tau_{31}=\tau_{13}
$$

for a total of only four quantities to define $\tau_{i j}$.

The resulting stochastic differential equations for the three components of the Lagrangian velocity are

$$
\begin{aligned}
d u_{1} & =\left\{-\frac{C_{0} \varepsilon}{2}\left[\Gamma_{11}\left(u_{1}-\bar{u}_{1}\right)+\Gamma_{13} u_{3}\right]+\frac{\partial \bar{u}_{1}}{\partial x_{3}} u_{3}+\frac{1}{2} \frac{\partial \tau_{13}}{\partial x_{3}}\right. \\
& \left.+\left[\frac{\partial \tau_{11}}{\partial x_{3}}\left(\Gamma_{11}\left(u_{1}-\bar{u}_{1}\right)+\Gamma_{13} u_{3}\right)+\frac{\partial \tau_{13}}{\partial x_{3}}\left(\Gamma_{13}\left(u_{1}-\bar{u}_{1}\right)+\Gamma_{33} u_{3}\right)\right] \frac{u_{3}}{2}\right\} d t \\
& +\delta_{1 j}\left(C_{0} \varepsilon\right)^{1 / 2} d W_{j}(t), \\
d u_{2} & =\left[-\frac{C_{0} \varepsilon}{2}\left(\Gamma_{22} u_{2}\right)+\frac{\partial \tau_{22}}{\partial x_{3}}\left(\frac{\Gamma_{22} u_{2} u_{3}}{2}\right)\right] d t+\delta_{2 j}\left(C_{0} \varepsilon\right)^{1 / 2} d W_{j}(t), \\
d u_{3} & =\left\{-\frac{C_{0} \varepsilon}{2}\left[\Gamma_{13}\left(u_{1}-\bar{u}_{1}\right)+\Gamma_{33} u_{3}\right]+\frac{1}{2} \frac{\partial \tau_{33}}{\partial x_{3}}\right. \\
& \left.+\left[\frac{\partial \tau_{13}}{\partial x_{3}}\left(\Gamma_{11}\left(u_{1}-\bar{u}_{1}\right)+\Gamma_{13} u_{3}\right)+\frac{\partial \tau_{33}}{\partial x_{3}}\left(\Gamma_{13}\left(u_{1}-\bar{u}_{1}\right)+\Gamma_{33} u_{3}\right)\right] \frac{u_{3}}{2}\right\} d t \\
& +\delta_{3 j}\left(C_{0} \varepsilon\right)^{1 / 2} d W_{j}(t) .
\end{aligned}
$$

The components of the inverse of $\tau_{i j}, \Gamma_{i j}$, as defined in Eq. (11a-b), are as follows (Barnett, 1990):

$$
\begin{aligned}
& \Gamma_{11}=\left(\tau_{11}-\tau_{13}^{2} / \tau_{33}\right)^{-1} \\
& \Gamma_{22}=\left(\tau_{22}\right)^{-1} \\
& \Gamma_{33}=\left(\tau_{33}-\tau_{13}^{2} / \tau_{11}\right)^{-1} \\
& \Gamma_{13}=\left(\tau_{13}-\tau_{11} \tau_{33} / \tau_{13}\right)^{-1}
\end{aligned}
$$

Note that $\Gamma_{22}$ is equal to the reciprocal of the Reynolds stress $\tau_{22}$. Also note that if $\tau_{13} \rightarrow 0, \Gamma_{13} \rightarrow 0$, $\Gamma_{11} \rightarrow\left(\tau_{11}\right)^{-1}$, and $\Gamma_{33} \rightarrow\left(\tau_{33}\right)^{-1}$. I appreciate the help of Brian Sawford who explained the meaning of the symbols for the inverse of a tensor in Thomson (1987) and Sawford and Guest (1988). There is coupling between these equations: between $u_{1}$ and $u_{3}$ in Eq. (12a), between $u_{2}$ and $u_{3}$ in Eq. (12b), and between $u_{1}$ and $u_{3}$ in Eq. (12c). 
For convenience, we give the components of the Reynolds stress tensor $\tau_{i j}$ in terms of more familiar symbols:

$$
\begin{aligned}
& \tau_{11}=\overline{u u}=\sigma_{u}^{2}, \\
& \tau_{22}=\overline{v v}=\sigma_{v}^{2}, \\
& \tau_{33}=\overline{w w}=\sigma_{w}^{2}, \\
& \tau_{13}=\overline{u w}=\tau_{u w},
\end{aligned}
$$

Here $u, v, w$ replace $u_{1}-\bar{u}_{1}, u_{2}, u_{3}$, the velocity fluctuations in the longitudinal, lateral, and vertical directions, respectively. The overbar indicates a mean quantity. $\sigma^{2}$ is both a velocity variance and a kinematic normal Reynolds stress. $\tau_{u w}$ is a Reynolds shear stress.

\section{Reduction for Diffusion in Two Dimensions}

For turbulent diffusion in only the lateral and vertical dimensions ( $x_{2}$ and $x_{3}$ or $y$ and $z$ ), we consider only the stochastic differential equations for $d u_{2}$ and $d u_{3}$ (or $d v$ and $d w$ ). Equation (12b) is unchanged; we repeat it here for convenience, making use of Eq. (13b),

$$
d u_{2}=\left[-\frac{C_{0} \varepsilon}{2}\left(\frac{u_{2}}{\tau_{22}}\right)+\frac{\partial \tau_{22}}{\partial x_{3}}\left(\frac{u_{2}}{\tau_{22}}\right) \frac{u_{3}}{2}\right] d t+\delta_{2 j}\left(C_{0} \varepsilon\right)^{1 / 2} d W_{j}(t)
$$

Equation (13c) is drastically changed because the quantities $u_{1}=\bar{u}_{1}$ and $\tau_{13}$ associated with longitudinal diffusion vanish. We use Eq. (13a) with $\tau_{13}=0$ to get the familiar

$$
d u_{3}=\left\{-\left(\frac{C_{0} \varepsilon}{2}\right) \frac{u_{3}}{\tau_{33}}+\frac{1}{2}\left(1+\frac{u_{3}^{2}}{\sigma_{w}^{2}}\right) \frac{\partial \tau_{33}}{\partial x_{3}}\right\} d t+\delta_{3},\left(C_{0} \varepsilon\right)^{1 / 2} d W_{j}(t)
$$

Equation (15b) is equivalent to Eq. (1a), the Langevin model first proposed by Wilson et al. (1983) for vertical diffusion in vertically inhomogeneous turbulence.

\section{Reduction for Diffusion in One Dimension}

For turbulent diffusion in only the vertical dimension $\left(x_{3}\right.$ or $z$ ), we are left with only the above stochastic differential equation for $d u_{3}$ (or $d w$ ), Eq. (15b). For convenience, we rewrite it using the more familiar symbols:

$$
d w=\left\{-\left(\frac{C_{0} \varepsilon}{2}\right) \frac{w}{\sigma_{w}^{2}}+\frac{1}{2}\left(1+\frac{w^{2}}{\sigma_{w}^{2}}\right) \frac{\partial \sigma_{w}^{2}}{\partial z}\right\} d t+\left(C_{0} \varepsilon\right)^{1 / 2} d W(t)
$$




\section{Random Displacement Models for Diffusion in Gaussian Turbulence}

We begin with the random displacement equivalent of Eqs. (7a-b),

$$
d x_{i}=a_{i}(\mathbf{x}, t) d t+b_{i j}(\mathbf{x}, t) d W_{j}(t)
$$

where $x_{i}$ is displacement; $\mathbf{a}$ and $\mathbf{b}$ are functions of $\mathbf{x}$ and $t$, and the $d W_{j}(t)$ are increments of a vector-valued Wiener process with independent increments. As with Eqs. (7a-b), the mean of $d W_{j}$ is zero and the variance is $d t$; the increments of $d W_{j}$ at different times, or at the same time with $i \neq j$ are independent. The analog to Eq. (8) for Eq. (17) is

$$
K_{i j}=\frac{1}{2} b_{i k} b_{k j}
$$

where $K_{i j}$ is the eddy diffusivity tensor. It is often assumed that $K_{i j}$ is diagonal;

$$
K_{i j}=0 \text { if } i \neq j .
$$

The solution for $a_{i}$ is

$$
a_{i}=\partial K_{i j} / \partial x_{j}
$$

Then

$$
d x_{i}=\frac{1}{2}\left(\partial b_{i k} b_{k j} / \partial x_{j}\right) d t+b_{i j} d W_{j}(t)
$$

For our assumed stationary turbulent flow that is horizontally homogeneous, the eddy diffusivities with respect to Eq. (18b) are (no summation)

$$
K_{i i}=2 \tau_{i i}^{2} / C_{0} \varepsilon
$$

where

$$
\begin{aligned}
& \tau_{11}=\sigma_{u}^{2} \\
& \tau_{22}=\sigma_{v}^{2} \\
& \tau_{33}=\sigma_{w}^{2}
\end{aligned},
$$

\section{Langevin Models for Diffusion in Non-Gaussian Turbulence}

\section{Model for One-Dimensional Diffusion in the Convective Boundary Layer}

In Part II we followed, in gुeneral, the chronological development of the model. Here we will, in a sense, work backward in time. We will start with a complex Langevin model for diffusion in non-Gaussian inhomogeneous turbulence. We will simplify it by reducing it to it's form for Gaussian inhomogenecus turbulence. 
We will consider turbulent diffusion in only one spatial dimension for two reasons. First, it simplifies the presentation. Second, there are, at present, insufficient criteria for defining a unique form of the Lagrangian equation for diffusion in more than one dimension (Thomson, 1987; Sawford and Guest, 1988).

The Langevin equation can be written in generalized form as the sum of a deterministic term and a random or stochastic term:

$$
d w=a(z, w, t) d t+b(z, w, t) d W(t)
$$

where $t$ is time, $w$ is the Lagrangian velocity, and $z$ is the spatial coordinate. The deterministic term is $[a(z, w, t) d t]$ and the stochastic tr.rm is $[\zeta(z, w, t) d W(t)]$. We will consider several different forms of the deterministic term in subsequent sections. Different forms, some with non-Gaussian forcing, have been proposed for the stochastic term, but it has been established that the random forcing must be Gaussian (Thomson, 1987, Sec. 2.3; see also van Dop et al., (1985), Sec. V, and Sawford, 1986, Sec. IV). Therefore $d W(t)$ is an incremental Wiener process with

$$
\langle d W(t)\rangle=0,\left\langle[d W(t)]^{2}\right\rangle=d t
$$

It follows that

$$
b^{2}=2 \sigma_{w}^{2} / \tau_{L}=C_{0} \varepsilon
$$

where $\sigma_{w}^{2}$ is the Eulerian variance of $w, \tau_{L}$ is a Lagrangian velocity decorrelation timescale, $C_{0}$ is a universal constant associated with the Lagrangian velocity structure function, and $\varepsilon$ is the mean rate of dissipation of turbulent kinetic energy (Thomson, 1987). As indicated by Eq. (24), $\sigma_{w}, \tau_{L}$, and $\varepsilon$ in Eq. (26) are, in general, function of $z, w$, and $t$. We will assume the turbulence to be stationary so $a=a(z, w)$ and $b=b(z, w)$ in the following.

Baerentsen and Berkowicz (1984) approximated the non-Gaussian turbulence in the convective boundary layer (CBL) by means of the sum of two Gaussian distributions:

$$
P_{a}(w \mid z)=\frac{1}{\sqrt{2 \pi}}\left\{\frac{\lambda_{1}}{\sigma_{1}} \exp \left[-\frac{1}{2}\left(\frac{w-m_{1}}{\sigma_{1}}\right)^{2}\right]+\frac{\lambda_{2}}{\sigma_{2}} \exp \left[-\frac{1}{2}\left(\frac{w-m_{2}}{\sigma_{2}}\right)^{2}\right]\right\} .
$$

Here $P_{a}(w \mid z)$ is the probability distribution function (PDF) for the Lagrangian velocity $w$ at elevation $z, \lambda_{1}$ is the probability of occurrence of updrafts, and $\lambda_{2}$ is that for downdrafts. The parameters $\lambda_{1}$ and $\lambda_{2}$ can also be interpreted as the respective fractional areas occupied by updrafts and downdrafts. With $i=1,2$ (no summation), the mean Lagrangian velocity is

$$
m_{i}=\left\langle w_{i}\right\rangle
$$

and the velocity variance is

$$
\sigma_{i}^{2}=\left\langle w_{i}^{2}\right\rangle
$$

The parameters $\lambda, m$, and $\sigma$ are function of $z$. The PDF for the updrafts is a Gaussian distribution centered at $w=m_{1}$ and that for the downdrafts is a Gaussian distribution centered at $w=$ $m_{2}$. We note that $m_{1}>0>m_{2}$. For a given value of $z, P_{a}(w \mid z)$ is defined by six parameters: $\lambda_{1}, \lambda_{2}, m_{1}, m_{2}, \sigma_{1}, \sigma_{2}$. 
Baerentsen and Berkowicz equated the zeroth through third moments of $P_{a}$,

$$
\left\langle w^{n}\right\rangle=\int_{-\infty}^{\infty} w^{n} P_{a}(w \mid z) d z
$$

(with $n=0$ to 3 ), to the corresponding moments for the CBL. Evaluation of these moments using Eqs. (27)-(29) gives

$$
\begin{gathered}
\lambda_{1}+\lambda_{2}=1, \\
\lambda_{1} m_{1}+\lambda_{2} m_{2}=0, \\
\lambda_{1}\left(m_{1}^{2}+\sigma_{1}^{2}\right)+\lambda_{2}\left(m_{2}^{2}+\sigma_{2}^{2}\right)=\sigma_{w}^{2}, \\
\lambda_{1}\left(m_{1}^{3}+3 m_{1} \sigma_{1}^{2}\right)+\lambda_{2}\left(m_{2}^{3}+3 m_{2} \sigma_{2}^{2}\right)=\left\langle w^{3}\right\rangle .
\end{gathered}
$$

The zeroth moment Eq. (31a) states that the "marked" particle is either in an updraft or a downdraft. The first moment Eq. (318b) can be interpreted as the mass conservation equation for constant-density flow (flow up - flow down). The left sides of the equations for the second (31c) and third (31d) moments are set equal to values based on measurements of $\sigma_{w}^{2}$ and $\left\langle w^{3}\right\rangle$, respectively.

Equations (31a) and (31b) can be combined to give

$$
\lambda_{1}=\left(1-m_{1} / m_{2}\right)^{-1}
$$

and

$$
\lambda_{2}=\left(1-m_{2} / m_{1}\right)^{-1}
$$

Weil (1990) introduced the parameter $R$ which is defined as

$$
R=\sigma_{1} / m_{1}=-\sigma_{2} / m_{2}
$$

Weil made a more general assumption than did Baerentsen and Beckowicz who used $R=1$. The statement made by Eq. (33) is an assumption which seems to be consistent with observations. Weil also introduced the skewness $S$,

$$
S=\left\langle w^{3}\right\rangle / \sigma_{w}^{3}
$$

and the parameters $\alpha$ and $\beta$ where

$$
\propto=\left(1+R^{2}\right)\left(1+3 R^{2}\right)^{-1}
$$

and

$$
\beta=1+R^{2} .
$$

Weil obtained the following solutions from Eqs. (31c)-(36):

$$
\frac{m_{1}}{\sigma_{w}}=\frac{1}{2}\left[\propto S+\left(\propto^{2} S^{2}+\frac{4}{\beta}\right)^{\frac{1}{2}}\right],
$$

and

$$
\frac{m_{2}}{\sigma_{w}}=\frac{1}{2}\left[\propto S-\left(\propto^{2} S^{2}+\frac{4}{\beta}\right)^{\frac{1}{2}}\right] \text {. }
$$


With data for values of $\sigma_{w}^{2}$ and $\left\langle w^{3}\right\rangle$ and an assumed value for $R$ (e.g., unity), all the parameters in Eq. (27) for the PDF of the CBL are determined.

Luhar and Britter (1989) and Sawford (Weil, 1990) independently derived the following Langevin equation for diffusion in the CBL:

$$
d w=a(z, w) d t+\left(C_{0} \varepsilon\right)^{\frac{1}{2}} d W(t)
$$

where

$$
a=-\frac{\sigma_{w}^{2}}{\tau_{L}}\left(\frac{Q}{P_{a}}\right)+\frac{\phi}{P_{a}}
$$

in which

$$
\begin{aligned}
Q & =\frac{\lambda_{1}\left(w-m_{1}\right)}{\sigma_{1}^{2}}\left\{\frac{1}{\sigma_{1} \sqrt{2 \pi}} \exp \left[-\frac{1}{2}\left(\frac{w-m_{1}}{\sigma_{1}}\right)^{2}\right]\right\} \\
& +\frac{\lambda_{2}\left(w-m_{2}\right)}{\sigma_{2}^{2}}\left\{\frac{1}{\sigma_{2} \sqrt{2 \pi}} \exp \left[-\frac{1}{2}\left(\frac{w-m_{2}}{\sigma_{2}}\right)^{2}\right]\right\}
\end{aligned}
$$

and

$$
\begin{aligned}
\phi & =\left[\sigma_{1}^{2} \frac{d \lambda_{1}}{d z}+\frac{\lambda_{1}}{2}\left(\frac{w^{2}}{\sigma_{1}^{2}}+1\right) \frac{d \sigma_{1}^{2}}{d z}\right]\left\{\frac{1}{\sigma_{1} \sqrt{2 \pi}} \exp \left[-\frac{1}{2}\left(\frac{w-m_{1}}{\sigma_{1}}\right)^{2}\right]\right\} \\
& +\left[\sigma_{2}^{2} \frac{d \lambda_{2}}{d z}+\frac{\lambda_{2}}{2}\left(\frac{w^{2}}{\sigma_{2}^{2}}+1\right) \frac{d \sigma_{2}^{2}}{d z}\right]\left\{\frac{1}{\sigma_{2} \sqrt{2 \pi}} \exp \left[-\frac{1}{2}\left(\frac{w-m_{2}}{\sigma_{2}}\right)^{2}\right]\right\} \\
& -\frac{1}{2}\left\{\left[\frac{d}{d z}\left(\lambda_{1} m_{1}\right)\right] \operatorname{erf}\left(\frac{w-m_{1}}{\sigma_{1} \sqrt{2}}\right)+\left[\frac{d}{d z}\left(\lambda_{2} m_{2}\right)\right] \operatorname{erf}\left(\frac{w-m_{2}}{\sigma_{2} \sqrt{2}}\right)\right\} .
\end{aligned} .
$$

We see that $Q$ and $\phi$ contain elements of $P_{a}$ as written in Eq. (27):

$$
P_{a}=\frac{1}{\sqrt{2 \pi}}\left\{\frac{\lambda_{1}}{\sigma_{1}} \exp \left[-\frac{1}{2}\left(\frac{w-m_{1}}{\sigma_{1}}\right)^{2}\right]+\frac{\lambda_{2}}{\sigma_{2}} \exp \left[-\frac{1}{2}\left(\frac{w-m_{2}}{\sigma_{2}}\right)^{2}\right]\right\} \text {. }
$$

\section{Appendix: Reduction to Gaussian Turbulence}

To review, the absolute values of the velocities in the updrafts in the convective boundary layer are greater than those of the downdrafts. With reference to Eqs. (31a-b),

$$
\begin{gathered}
m_{1}>0>m_{2}, \\
m_{1}>\left|m_{2}\right|,
\end{gathered}
$$

and

$$
\lambda_{2}>\lambda_{1}
$$


Let us assume a transition from unstable to neutral conditions. The convection will weaken and the mean updraft and downdraft velocities will approach zero:

$$
m_{1} \rightarrow 0(+) \text { and } m_{2} \rightarrow 0(-)
$$

The $(+)$ and $(-)$ symbols indicate approaches to the limiting value of zero from the positive and negative sides, respectively. Furthermore, let the limits in Eq. (46) be approached in such a way that

$$
m_{2} / m_{1} \rightarrow-1
$$

As a consequence of Eqs. (46)-(47), Eqs. (32 a-b) take the limiting forms

$$
\lambda \rightarrow \frac{1}{2}(-) \text { and } \lambda_{2} \rightarrow \frac{1}{2}(t)
$$

If we apply the limiting solutions in Eqs. (46)-(48), we get

$$
\left\langle w^{3}\right\rangle \rightarrow 0
$$

from Eq. (31d) and

$$
\sigma_{1}^{2}+\sigma_{2}^{2} \rightarrow 2 \sigma_{w}^{2}
$$

from Eq. (31c). It is logical to interpret Eq. (50) to mean that, in the limit,

$$
\sigma_{1}^{2}=\sigma_{2}^{2}=\sigma_{w}^{2}
$$

Therefore the limiting form of Eqs. (27) and (42) is the Gaussian PDF,

$$
P_{a}(w \mid z)=\frac{1}{\sigma_{w} \sqrt{2 \pi}} \exp \left[-\frac{1}{2}\left(\frac{w}{\sigma_{w}}\right)^{2}\right]
$$

In this limiting case, the two terms on the right side of Eq. (40) approach each other in value so

$$
Q / P_{a}=w / \sigma_{w}^{2}
$$

Similarly, in Eq. (41) the gradients of $\lambda$ and $\lambda_{2}$ vanish as do the error functions. As a result,

$$
\frac{\phi}{P_{a}}=\frac{1}{2}\left(\frac{w^{2}}{\sigma_{w}^{2}}+1\right) \frac{d \sigma_{w}^{2}}{d z}
$$


Then from Eqs. (38), (39), (53), and (54) we get the Langevin equation for Gaussian inhomogeneous turbulence that was formulated by Wilson et. al. (1983) and rigorously derived by Thomson (1984):

$$
d w=\left[-\frac{w}{\tau_{L}}+\frac{1}{2}\left(\frac{w^{2}}{\sigma_{w}^{2}}+1\right) \frac{d \sigma_{w}^{2}}{d z}\right] d t+\left(C_{0} \varepsilon\right)^{1 / 2} d W(t)
$$

We note from Eqs. (38)-(39) and (55) that $\left\{-\left(\sigma_{w}^{2} / \tau_{L}\right)\left(Q / P_{a}\right)\right\}$ is related to the "fading memory" or decorrelation term and that $\phi / P_{a}$ is related to the "drift" correction.

We have demonstrated how the Langevin model for non-Gaussian inhomogenous turbulence reduces to the more familiar model for Gaussian inhomogeneous turbulence. This reduction is explicitly stated in Luhar and Britter (1989) and is implied in Weil (1990), but no details are presented.

The Langevin model defined by Eqs. (38)-(42) was developed explicitly for diffusion in the $\mathrm{CBL}$, but the CBL is not the only case of non-Gaussian turbulence in the atmosphere. van Dop et. al. (1985) commented, "It is however, generally observed that in inhomogeneous turbulent flows, the turbulence is not Gaussian." Pope (1987), in a little-known paper that is similar in intent to the much-cited Thomson (1987), strongly criticizes the assumption of Gaussian inhomogeneous turbulence as being unphysical. It is therefore in order to consider the use of Eqs. (38)-(42) for more cases than the CBL.

On the cther hand, the computing costs associated with Eqs. (38)-(42) may prove to be substantially greater than those with Eq. (55). In view of the simplifications for $Q / P_{a}$ from Eqs. (40) and (42) and Eqs. (42) and (51) and those for $\phi / P_{a}$ from Eqs. (41) and (42) to Eqs. (53) and (54), it will be appropriate to determine the values of $R$ (Eq. (33)) and $S$ (Eq. (11)) for which the use of the simpler model (Eq. (55)) is appropriate.

The above two paragraphs illustrate a great advantage of the Langevin model. We can change the model and its inputs to examine the effects of different turbulence properties on diffusion: e.g., Gaussian vs. non-Gaussian, homogeneous vs. inhomogeneous, etc.

\section{Extension to Three Dimensional Diffusion}

At the present time, there is no known published extension of the Luhar-Britter (1989) and Sawford/Weil (1990) model for one-dimensional diffusion in non-Gaussian turbulence to three dimensions.

\section{Parameterization of Turbulence Statistics for Use as Model Inputs}

\section{Introduction}

The principal inputs to the random-walk model based on the Langevin equation for turbulent dispersion in the atmosphere are (1) the variance of the turbulent velocity fluctuations and (2) either (a) the local decorrelation time scale or (b) the rate of dissipation of turbulent kinetic energy. In 
general, these inputs are functions of time, space, and atmospheric stability. In practice, these inputs are assumed to be relatively weak functions of both time and the horizontal coordinates so they are parameterized in terms of only the vertical coordinate and the atmospheric stability. (Very little has been done to apply this model to turbulent disperison in three dimensions.) The published parameterizations for vertical dispersion do not give continuous values for the model inputs across the transitions from one atmospheric height-and-stability regime to another (e.g., from the unstable free convection layer to the mixed layer).

In this memorandum, we will use the scaling parameters and diagram of Holtslag and Nieuwstadt (1986) for dividing the idealized atmospheric boundary layer into distinct scaling regimes as functions of the dimensionless height $z / h$ and the dimensionless stability parameter $h / L$ where $z$ is the vertical coordinate, $h$ is the height of the turbulent boundary layer, and $L$ is the Monin-Obukhov scaling length. We will assume stationary, horizontally homogeneous turbulence statistics. We will first consider dispersion in only the vertical dimension. We will propose parameterizations of the variance $\sigma_{w}^{2}$ and the dissipation rate $\varepsilon$ that are continuous functions of $z, h$, and $L$. We will also discuss known deficiencies of the proposals. We will then consider the horizontal variances $\sigma_{u}^{2}$ and $\sigma_{v}^{2}$ and the shear stress $\tau_{u w}$ in a similar manner.

\section{Basic Scaling Parameters}

The scaling velocity for turbulence in the near-neutral and stable boundary layers is defined by the equation

$$
-\langle u w\rangle_{0}=u_{*}^{2}
$$

where $u$ and $w$ are, respectively, the longitudinal and vertical turbulent velocity fluctuations, $\langle u w\rangle_{0}$ is the Reynolds-averaged turbulent kinematic shear stress at the surface, and $u *$ is the friction velocity. A companion relation is

$$
-\langle w \theta\rangle_{0}=u_{*} \theta_{*}
$$

where $\theta$ is the turbulent fluctuation of the temperature, $\langle w \theta\rangle_{0}$ is the Reynolds-averaged turbulent kinematic temperature flux at the surface, and $\theta$, is a temperature scale for turbulent heat transfer. A combination of the buoyancy parameter $g / T$ (where $g$ is the acceleration of gravity and $T$ is the absolute temperature), $u_{*}$, and $\theta$, defines the Monin-Obukhov length scale:

$$
L=u_{*}{ }^{2} T / k g \theta_{*}=-u_{*}^{3} T / k g\langle w \theta\rangle_{0}
$$

where $k$ is the von Karman constant $(\approx 0.4)$. The length scale $L$ is more convenient to use than the quantity $g / T$; furthermore $L>0$ in stable conditions and $L<0$ in the unstable boundary layer.

The product of $g / T, u_{*}$, and $\theta_{*}$ is

$$
B=-g u_{*} \theta_{*} / T=\langle w \theta\rangle_{0} g / T
$$

where $B$ is the surface rate of buoyancy production $\left(\langle w \theta\rangle\right.$ is positive) or destruction $\left(\langle w \theta\rangle_{0}\right.$ is negative) of turbulent kinetic energy. The shear-stress production rate of turbulent kinetic energy is

$$
S=u_{*}^{3} / k z
$$


where $z$ is the height above the surface. The minimum value of $z$ is $z_{0}$, the surface roughness length. The ratio of the rates of buoyant to shear-stress production of turbulent kinetic energy is from Equations (58)-(60),

$$
B / S=-z / L
$$

This shows that the effect of buoyancy approaches zero as $|z / L| \rightarrow 0$, and the effect of shear stress becomes negligible for sufficiently large values of $|z / L|$.

In stable boundary layers $(L>0)$, Eqs. (58)-(61) indicate that the destruction of turbulent kinetic energy by negative buoyancy will limit the height at which turbulence can be maintained. On the other hand for unstable boundary layers $(L<0)$, these equations imply that $u_{*}$ is not an appropriate scaling velocity for turbulence if the effect of shear stress is negligible. A more appropriate scaling velocity is defined by the equation

$$
B H=\langle w \theta\rangle_{0} g h / T=w_{*}^{3}
$$

where $h$ is the height of the unstable turbulent boundary layer and $w_{*}$ is the convective velocity scale. From Equations (58) and (62),

$$
k\left(w_{*} / u_{*}\right)^{3}=-h / L
$$

Further discussion of the above is given in van Ulden and Holtslag (1985) and Holtslag and Nieuwstadt (1986).

\section{Vertical Velocity Variance $\sigma_{w}^{2}$}

We need a parameterization for $\sigma_{w}^{2}$ that is continuous for all elevations within the turbulent boundary layer $\left(z_{0}<z<h\right)$ and the full range of atmospheric conditions from unstable to stable $\left(-\infty<L^{-1}<\infty\right)$. We note that $u_{*}$ and $w_{*}$ are scaling velocities for different regimes in the turbulent boundary layer so we assume a general relationship of the form

$$
\sigma_{w}^{2}=a u_{*}^{2}+b w_{*}^{2}
$$

where $a$ and $b$ are functions to be determined. From Eqs. (63)-(64),

$$
\left(\sigma_{w} / u_{*}\right)^{2}=a+b(h /-k L)^{2 / 3} .
$$

We propose that the term with $b$ be negligible or zero in near-neutral and stable conditions for which $u_{*}$ is the appropriate scaling velocity. We also propose that the term with $a$ be negligible in very unstable conditions for which $w_{*}$ is the proper scaling velocity. The following meets these criteria:

$$
\left(\frac{\sigma_{w}}{u_{*}}\right)^{2}=C_{1}\left(1-\frac{z}{h}\right)^{3 / 2}+C_{2}\left[\left(\frac{z}{h}\right)\left(\frac{h}{-L}\right)\right]^{2 / 3}\left(1-C_{3} \frac{z}{h}\right)^{n}
$$

where, for the present,

$$
\begin{aligned}
C_{1} & =1.6 \\
C_{2} & =0 \text { if } L>0 \text { and } C_{2}=2.4 \text { if } L<0, \\
C_{3} & =0.8 \\
n & =2 .
\end{aligned}
$$


The term with $C_{1}$ is a modification of Eq. (38) for the stable boundary layer in Nieuwstadt (1984) and that with $C_{2}$ is based on Equation (13) for the convective boundary layer in Lenschow et al. (1980).

Equation (66) with $C_{2}=0$ was derived by Nieuwstadt (1984) for the stable boundary layer. He used $C_{1}=1.06$ to get a reasonable fit to both his Cabauw tower data and the data of Caughey et al., (1979) (see Nieuwstadt's Fig. 11). Our value of $C_{1}=1.6$ gives a good fit to the Cabauw data and, for small values of $z / h$, gives a better fit to most data for the near-neutral and neutral surface layer (to be discussed later).

For the unstable surface layer $(0<z / h<0.1)$, the quantities $(1-z / h)$ and $\left(1-C_{3} z / h\right) \approx 1$ so we approximate our Eq. (66) as

$$
\left(\frac{\sigma_{w}}{u_{*}}\right)^{2}=1.6+2.4\left(\frac{z}{-L}\right)^{2 / 3}
$$

which is similar to Eq. (7) in Panofsky et al., (1977):

$$
\left(\frac{\sigma_{w}}{u_{*}}\right)^{2}=1.4+2.9\left(\frac{z}{-L}\right)^{2 / 3}
$$

Equations (65), (67), and (68) are consistent with the local free convection prediction for the very unstable surface layer that $\left(\sigma_{w} / u_{*}\right)^{2}$ is proportional to $(z /-L)^{2 / 3}$ (see Fig. 1 in Wyngaard et al., 1971). We use $C_{2}=2.4$ instead of $C_{w}=2.9$ as in Panofsky et al., because their data for $\sigma_{w} / u_{*}$ are significantly higher than data sets from Kansas and Sweden (see text on p. 1959 and Fig. 16 in Högström, 1990). Our value of $C_{2}=2.4$ also gives a better fit to data for the convective boundary layer or mixed layer as described below.

We have noted that the term with $C_{2}$ in Eq. (66) is based on Eq. (13) for the convective boundary layer in Lenschow et al. (1980):

$$
\left(\frac{\sigma_{w}}{w_{*}}\right)^{2}=1.8\left(\frac{z}{h}\right)^{2 / 3}\left(1-0.8 \frac{z}{h}\right)^{2}
$$

This equation is a fit to data obtained from flight measurements. According to Weil (1990) p. 513. the average value of the ration $u_{*} / w_{*}$ for these data was 0.25 . From Eq. (63) with $k=0.4$. this corresponds to $h / L=-25.6$, a condition in which the effect of the shear stress is negligible, except very close to the surface. Equation (69) does not account for the shear-stress effect, unlike Eq. (36a) in Weil which is

$$
\left(\frac{\sigma_{w}}{w_{*}}\right)^{2}=\left[\left(\frac{u_{*}}{w_{*}}\right)^{3}\left(1.6-\frac{z}{h}\right)^{3 / 2}+1.2 \frac{z}{h}\left(1-0.98 \frac{z}{h}\right)^{3 / 2}\right]^{2 / 3}
$$

As in Eq. (66), the first term in the right accounts for the shear-stress effect and the second for the convective $\epsilon$ ffect. Weil fitted his equation to both the flight data of Lenschow et al. and the large eddy imulation (LES) data of Moeng and Wyngaard $(1984,1989)$. The ratio $u * / w *$ in these LES was 0.3 , which corresponds to $h / L=-14.8$. We chose to use Eq. (66) instead of Eq. (70) in order 
to give continuous values of $\sigma_{w} / u_{*}^{2}$ for all ranges of $z / h$ and $h / L$. However, we will use Eq. (70) as a reference in selecting the value for $C_{2}$ in Eq. (66). From Eqs. (63) and (69) with $k=0.4$,

$$
\left(\frac{\sigma_{w}}{u_{*}}\right)^{2}=3.3\left[\left(\frac{h}{-L}\right)\left(\frac{z}{h}\right)\right]^{2 / 3}\left(1-0.8 \frac{z}{h}\right)^{2} .
$$

Here $C_{2}$ (see Eq. (66)) is equal to 3.3 instead of 2.4 as in Eqs. (66) and (67) and 2.9 as in Eq. (68). Our value of $C_{2}=2.4$ in Eq. (11) gives greater values for $\left(\sigma_{w} / u_{*}\right)^{2}$ than Eq. (70) for $z / h<0.4$ and lesser values for $z / h=0.4$ and greater, assuming $u_{*} / w_{*}=0.3$.

In summary, Eq. (66) gives continuous values for $\left(\sigma_{w} / u_{*}\right)^{2}$ for all elevations within the turbulent boundary layer and the full range of atmospheric stability conditions. On the stable side, it is a slight modification to Nieuwstadt's (1984) equation. On the unstable side, it is the sum of the modified Nieuwstadt equation and a modification of the equation of Lenschow et al. (1980). In the surface layer, it has the correct asymptotic form for the free convection layer. The present values of $C_{1}, C_{2}, C_{3}$ and $n$ are the results of a first attempt to approximate field and numerical data. The second attempt will involve more exhaustive attempts to fit the available data from both nature and simulations. Additional work will probably include making a series of large eddy simulations to generate needed data. The final test will be the use of Eq. (66) with our random-walk dispersion model based on the Langevin equation.

\section{Rate of Dissipation of Turbulence Kinetic Energy $\varepsilon$}

The parameterization for $\varepsilon k h / u_{*}^{3}$ is more difficult than it is for $\left(\sigma_{w} / u_{*}\right)^{2}$. In part, this is because $\varepsilon k h / u_{*}^{3}$ does not follow similarity laws but is determined from the turbulent kinetic energy budget. In addition, there are fewer sources of data for $\varepsilon$, field experiments as well as numerical simulations.

We propose a pair of parameterizations for $\varepsilon k h / u_{*}^{3}$. For the neutral to stable atmospheres,

$$
\frac{\varepsilon k h}{u_{*}^{3}}=\frac{h}{z}\left[1+C_{4}\left(\frac{z}{h}\right)\left(\frac{h}{L}\right)\right]\left(1-C_{5} \frac{z}{h}\right)^{m} .
$$

For the unstable to neutral case,

$$
\frac{\varepsilon k h}{u_{*}^{3}}=\frac{h}{z}\left\{\left[1+C_{6}\left(\frac{z}{h}\right)\left(\frac{h}{-L}\right)\right]\left(1-C_{5} \frac{z}{h}\right)^{m}\right\}+C_{7} \frac{h}{-L} .
$$

For the present,

$$
\begin{aligned}
C_{4} & =3.7, \\
C_{5} & =0.85 \\
C_{6} & =0.75 \\
C_{7} & =0.3, \\
m & =3 / 2
\end{aligned}
$$

The first parameterization is from Brost and Wyngaard (1978). The second is an ad hoc relation that was constructed as described below. 
Brost and Wyngaard (1978) studied the stably stratified boundary layer with a second-order turbulence model in which the rave of dissipation of turbulent kinetic energy was assumed to be proportional to the $\frac{3}{2}$-power of the turbulent kinetic energy divided by a length scale given by an height-dependent interpolation formula. They fitted Eq. (72) with the indicated constants to the dissipation rate calculated with their model. Lenschow el at. (1988) compared the predictions of this equation with their flight data as well as those of Caughey et al. (1979) and concluded "The agreement is generally pretty good." However, the data points have considerable scatter about the value given by Eq. (72). Mason and Derbyshire (1990) compared the Brost and Wyngaard results for $\varepsilon$ with theirs from large eddy simulations. They found good agreement for the stable case (for which the Brost and Wyngaard model was designed) and poor agreement for the neutral case. We will use Eq. (72) in the absence of anything better for the neutral-to-stable boundary layer.

We know of no counterpart to Eq. (72) for the unstable-to-neutral case. We start our construction of Eq. (73) with Eq. (20) in Wyngaard and Cote (1971) for the unstable surface layer,

$$
\frac{\varepsilon k z}{u_{*}^{3}}=\left(1+0.5 \frac{z}{-L}\right)^{3 / 2}
$$

The power-series expansion of Eq. (74) is

$$
\frac{\varepsilon k z}{u_{*}^{3}}=1+0.75 \frac{z}{-L}+\ldots
$$

This is a fair approximation to the curves for both the Kansas data (Wyngaard and Cote) and the Swedish data for the unstable-to-neutral surface layer plotted in Fig. 3 of Högströrn (1990). However, Eq. (75) is not in accordance with Högström's finding that the local rate of dissipation of turbulent kinetic energy in the neutral surface layer is about $20 \%$ greater than its local rate of production, that is, $\varepsilon k z / u_{*}^{3}=1.24$ instead of 1 in neutral conditions. We will keep this in mind, but for the present we will use the traditional $\varepsilon k z / u_{*}^{3}=1$.

We ensure continuous values for $\varepsilon k z / u_{*}^{3}$ for $0 \leq z / h \leq 1$ across the unstable-to-stable transition if we use the same $z / h$ function with $C_{5}$ and $m$ as in Eq. (72):

$$
\frac{\varepsilon k z}{u_{*}^{3}}=\left(1+0.75 \frac{z}{-L}\right)\left(1-0.85 \frac{z}{h}\right)^{3 / 2}
$$

Finally, as indicated in Fig. 5 of Luhar and Britter (1989) and Fig. 14d of Weil (1990), $s h / w_{*}^{3}$ is to a good approximation, approximately constant with height throughout much of the convective boundary layer. Therefore, to account for the buoyancy effect on $\varepsilon$,

$$
\frac{\varepsilon k z}{u_{*}^{3}}=\left(1+0.75 \frac{z}{-L}\right)\left(1-0.85 \frac{z}{h}\right)^{3 / 2}+0.3 \frac{z}{-L}
$$

This is equivalent to Eq. (73).

The refinement-perhaps replacement-of Eq. (72) and (73) will involve the same efforts described above for $\left(\sigma_{w} / u_{*}\right)^{2}$ : comparisons with existing data, generation of needed additional data, and use in our random walk model. 


\section{Skewness of the Vertical Velocity $\left\langle w^{3}\right\rangle / \sigma_{w}^{3}$}

The following is proposed for the dimensionless third moment of the vertical velocity fluctuations in the convective boundary layer $(h / L<0)$ :

$$
\frac{\left\langle w^{3}\right\rangle}{w_{*}^{3}}=C_{8}\left(1-\frac{z}{h}\right)
$$

This is the form given by Eq. (17) in Sorbjan (1990) and Eq. (36b) in Weil (1990). In Sorbjan, $C_{8}=0.5$, and $C_{8}=0.84$ in Weil. The two parts of Eq. (2.10) in Sawford and Guest (1987) combine to give $C_{8}=0.92$. In their paper, the right side of Eq. (78) is multiplied by a function of $z / h$ that is greater than unity if $z / h<0.3$ and less than unity if $z / h>0.3$. For the present, we will use $C_{8}=0.84$ for $h / L<0$ and $C_{8}=0$ for $h / L \geq 0$.

The combination of Eqs. (63) and (78) gives

$$
\frac{\left\langle w^{3}\right\rangle}{u_{*}^{3}}=\frac{0.84}{k}\left(\frac{h}{-L}\right) \frac{z}{h}\left(1-\frac{z}{h}\right)
$$

Equation (78) was developed for $h / L \ll 0$. As $h /-L \rightarrow 0$, corresponding to a change from unstable toward neutral conditions, $\left\langle w^{3}\right\rangle / u_{*}^{3} \rightarrow 0$. Therefore, with the above values of $C_{8}$, Eqs. (78) and (79) give continuous values for $\left\langle w^{3}\right\rangle$ over the full range of atmospheric conditions.

A comment by Ric Cederwall gave me the hint that led to the simple development from Eq. (78) to Eq. (79).

We should note that Eq. (79) is not in agreement with Table 2 on p 1969 in Högström (1990) where, for the neutral atmospheric surface layer, $\left\langle w^{3}\right\rangle / u_{*}^{3}=0.3 \pm 0.03$. Clearly, this is a matter that need further investigation. However, we do not presently have enough additional information to define non-Gaussian turbulence for a Langevin dispersion model for other than the unstable convective boundary layer (see Luhar and Britter (1989) and Weil (1990)).

\section{Horizontal Velocity Variances $\sigma_{u}^{2}$ and $\sigma_{v}^{2}$}

We propose the following for the variances $\sigma_{u}^{<}$and $\sigma_{v}^{2}$ of the turbulent velocity fluctuations in the horizontal plane:

$$
\left(\frac{\sigma_{u}}{u_{*}}\right)^{2}=\left(\frac{\sigma_{v}}{u_{*}}\right)^{2}=C_{9}\left(1-\frac{z}{h}\right)^{p}+C_{10}\left(\frac{z}{-L}\right)^{2 / 3}
$$

where, for the present,

$$
\begin{aligned}
C_{9} & =4.5 \\
C_{10} & =0 \text { if } L \geq 0 \text { and } C_{10}=0.6 \text { if } L \leq 0 \\
p & =3 / 2
\end{aligned}
$$

Here the variance for the longitudinal fluctuation $\sigma_{u}^{2}$ is assumed to be equal to that in the lateral direction $\sigma_{v}^{2}$. There are data, including those in Table 2 of Högström (1990), that indicate that $\sigma_{u}^{2}>\sigma_{v}^{2}$, but we will follow the reference cited below in which $\sigma_{u}^{2}=\sigma_{v}^{2}$. 
We note that Eq. (80) has the same form as Eq. (66) with $C_{3}=0$ and/or $n=0$. The justification for Eq. (80) is similar to that for Eq. (66). The term with $C_{9}$ is a modification of Eq. (30) for the stably stratified boundary layer in Lenschow et al. (1988). They use $p=7 / 4$ but admit that they cannot distinguish between $p=3 / 2$ and $p=7 / 4$ in fitting their data. The use of $p=3 / 2$ is consistent with Nieuwstadt's (1984) work which was used in the development of Eq. (66).

For the unstable surface layer $(0<z / h<0.1)$, Eq. (80) approximates Eq. (5) in Panofsky et al. (1977):

$$
\left(\frac{\sigma_{u}}{u_{*}}\right)^{2}=\left(\frac{\sigma_{v}}{u_{*}}\right)^{2}=4+0.6\left(\frac{z}{-L}\right)^{2 / 3}
$$

Panofsky et al. note that $\sigma_{u}$ and $\sigma_{v}$ become independent of $u_{*}$ and scale with $w_{*}$ for large negative values of $z / L$. We demonstrate this by combining Eqs. (63) and (81) to give, with $k=0.4$,

$$
\left(\frac{\sigma_{u}}{w_{*}}\right)^{2}=\left(\frac{\sigma_{v}}{u_{*}}\right)^{2}=4\left(\frac{u_{*}}{w_{*}}\right)^{2}+0.33
$$

For sufficiently small values of $u * / w$. (i.e., sufficiently large negative values of $h / L$ ), Eq. (82) is approximately equal to Eq. (5) in Caughey and Palmer (1979):

$$
\left(\frac{\sigma_{u}}{w_{*}}\right)^{2}=\left(\frac{\sigma_{v}}{w_{*}}\right)^{2}=0.35
$$

In closing, we note that Eqs. (66) and (80) are somewhat consistent with some, but not all, of the trends of the data shown in Fig. 5 of Nicholls and Readings (1979). Their data are for the slightly unstable surface layer $(0<x / h<0.1)$ and the near neutral upper layer $(0.1<z / h<1)$ shown in Fig. 1. Their Class A data are for an average value of $h / L=-3.9$ and their Class B data are for an average value of $h / L=-1.5$.

\section{Shear Stress $\tau_{u w}$}

The realizability condition for $\tau_{u w}$ is given by Eq. (1b) in Schumann (1977):

$$
\tau_{u w}^{2} \leq \sigma_{u}^{2} \sigma_{w}^{2}
$$

The parameterization for $\tau_{u w}$ will have to meet this condition. That proposed is

$$
\frac{\tau_{u w}}{u *^{2}}=\left(1-\frac{z}{h}\right)^{3 / 2-q}
$$

where, for the present,

$$
\begin{aligned}
& q=0 \text { if } L \geq 0 \text { and } \\
& q=-\frac{h / L}{2}\left(1-\frac{h}{L}\right)^{-1} \text { if } L<0 .
\end{aligned}
$$

For neutral conditions $h / L=0$ and stable conditions $(h / L>0)$, Eq. (85) is identical to Eq. (36) in Nieuwstadt (1984). For very unstable conditions, $(h / L \ll 0)$,

$$
q \approx 1 / 2
$$

and

$$
\frac{\tau_{u w}}{u *^{2}} \approx 1-z / h
$$

as Wyngaard et al. (1974) found in their numerical simulations of the atmospheric boundary layer. 


\section{Finite-Difference Forms of the Models}

\section{The Ito Calculus for Stochastic Differential Equations}

We have mentioned the Ito Calculus for stochastic differential equations several times in these Notes. Ito's rules for differentiation have been cited with respect to the orders of terms in stochastic differential equations; i.e., $(d t)^{2}=0,[d W(t) d t]=0$, and $[d W(t)]^{2}=d t$. Ito's rule for integrating stochastic differential equations has been mentioned in the Introduction to Part I and defined in Part II, the Fokker-Planck Equation: Relation to the Langevin Equation. On the other hand, we applied the conventional integral calculus to a form of the Langevin equation in Part II, Diffusion in Stationary Homogeneous Turbulence. We have the question, "What determines whether the classical Riemann-Stieltjes Calculus or the Ito Calculus should be used in integrating a stochastic differential equation?"

This question is probably as old as the Ito Calculus which was first described in a series of papers Ito published more than forty years ago. Gray and Caughey (1965) noted that the literature had become divided into two schools of thought with two different sets of results for apparently identical problems. They stated that the differences come from the difference between a strictly defined mathematical problem and a mathematical approximation to a physical problem. They cited Doob (1953) Sec VI.3 for the :nathematical approach which, according to the note on p. 629, is based on the work of Ito, Kolmogorov, and Feller. Stratonovich (1966) prepared an alternative to Ito's interpretation of the stochastic integral which permits the use of the classical integral calculus. Obviously confusion and controversy persisted because van Kampen (1981a), "Ito versus Stratonovich," showed that it is possible to relate Ito-type and Stratonovich-type equations in such a way that they are equivalent; i.e., with the same initial condition they determine the same process. He also showed, as did Stratonovich, that the Stratonovich approach is more appropriate for modeling physical processes that are not Markovian; that is, processes with short (but not infinitely short) correlation times. The "Ito versus Stratonovich" theme is reviewed in van Kampen (1981b); see the listings for the "Ito-Stratonovich dilemma" in the index of that book.

We will use the nonlinear Langevin equation,

$$
d w(t)=a[z(t)] d t+b[z(t)] d W(t)
$$

where

$$
d z(t)=w(t) d t
$$

in describing the Ito and Stratonovich integrals. From van Kampen (1981b) p. 244: “...equation [86a] as it stands has no meaning" (emphasis in original). It is meaningful only when combined with rules for its interpretation. The Ito integral of Eq. (86a) is

$$
w(t+\Delta t)-w(t)=a[z(t)] \Delta t+b[z(t)] \Delta W(t)
$$

and the Stratonovich integral is

$$
w(t+\Delta t)-w(t)=a[z(t)] \Delta t+b\left[\frac{z(t)+z(t+\Delta t)}{2}\right] \Delta W(t)
$$


That is, the Ito integral uses the value of the coefficient $b$ before the jump and the Stratonovich integral gives equal weights to the values of $b$ before and after the jump. Note that the difference between Eqs. (87a) and (87b) vanishes if $b$ is constant; this is why we could apply the classical integral calculus in Part II as noted above. The definitions of differences between, and relations (under certain conditions) between the Ito and Stratonovich integrals are also discussed in Arnold (1974) Sec. 4.2 and Ch. 10, and in Gardiner (1983) Secs. 4.2.6, 4.3.1, 4.3.2, and 4.3.6.

Arnold, van Kampen, and Gardiner also show by means of the Fokker-Planck equation that the rules for transformation between the Ito and Stratonovich integrals lead to two distinct, Markov processes as solutions, which differ in the systemic (drift) behavior but not in the fluctuational behaviur (Arnold, p. 171). In other words, Eq. (86a) can be written in the Ito and Stratonovich forms in terms of the same $b[z(t)]$ but a different $a[z(t)]$.

We close this section with a quote from Arnold (1974) pp. 167-168: "The Ito integral] is not symmetric with respect to the variable $t . .$. However, it is just this lack of symmetry that leads to the simple formulas for the first two moments of the integral... and to the martingale property.... Furthermore, a stochastic differential equation [like Eq. (86a)] explained on this basis yields... a diffusion process as a solution. A disadvantage is that the calculus deviates from the familiar one. This disadvantage (together with all the advantages of Ito's integral that we have mentioned) is removed by Stratonovich's definition...."

\section{Example: Finite-Difference Equations for 1D Diffusion}

The finite-difference version of Eq. (16) for one-dimensional (vertical) diffusion, written as an Ito integral in accordance with Eq. (87a), is

$$
w_{i+1}=w_{i}+\left\{-\frac{C_{0}}{2} \frac{\varepsilon_{i}}{\left(\sigma_{w}^{2}\right)_{i}} w_{i}+\frac{1}{2}\left(1+\frac{w_{i}^{2}}{\left(\sigma_{w}^{2}\right)_{i}}\right)\left(\frac{\partial \sigma_{w}^{2}}{\partial z}\right)_{i}\right\} \Delta t_{i}+\left(C_{0} \varepsilon_{i} \Delta t_{i}\right)^{1 / 2}\left(\Delta W_{3}\right)_{i}
$$

where

$$
\begin{aligned}
w_{i} & =w\left(z_{i}, t_{i}\right) \\
\varepsilon_{i} & =\varepsilon\left(z_{i}, t_{i}\right) \\
\left(\sigma_{w}^{2}\right)_{i} & =\sigma_{w}^{2}\left(z_{i}, t_{i}\right) \\
\left(\frac{\partial \sigma_{w}^{2}}{\partial z}\right)_{i} & =\left.\frac{\partial \sigma_{w}^{2}}{\partial z}\right|_{\substack{t=z_{i} \\
s=x_{i}}}, \\
z_{i} & =z\left(t_{i}\right), \\
\Delta t_{i} & =t_{i+1}-t_{i}, \text { and } \\
\left(\Delta W_{3}\right)_{i} & =W_{3}\left(t_{i+1}\right)-W_{3}\left(t_{i}\right)
\end{aligned}
$$

Here we use the normalized Wiener process as in Eq. (68) in Part II so the variance of $\Delta W_{3}$ is unity and $W_{3}$ is dimensionless. Gardiner (1983) pp. 93-95 calls this the Cauchy-Euler method (it is analogous to the simple, first-order Euler method for Ordinary Differential Equations) for solving an Ito Stochastic Differential Equation, and discusses its solution. The evaluation of terms in Eq. (88) at the beginning of the time step (at $t_{i}$ and $z_{i}$ ) is needed to ensure existence and 
uniqueness of a solution. Existence and uniqueness of a solution can be shown if $\left(\Delta W_{3}\right)_{i}$, the random increment of the Wiener process, is independent of $w_{i}$. In turn, $\left(\Delta W_{3}\right)_{i}$ is independent of $w_{i}$ if (1) the deterministic term (the term with $\Delta t$ in eq (88)) is a nonanticipating function of $t$ (that is, the deterministic term evaluated at $t_{i}$ must be statistically independent of $\left(\Delta W_{3}\right)_{i}$, the future value of $\Delta W_{3}$ ) and (2) the initial value of $w, w_{0}$, is also a nonanticipating function of $t$.

\section{Summary}

Two Lagrangian stochastic models for turbulent diffusion are considered: the Langevin equation model and the random displacement model., The latter is the diffusion equation limit of the Langevin model and it is the Langrangian equivalent of the Eulerian eddy diffusivity model. Both stochastic differential equations have valid mathematical solutions for all times $t \geq 0$, but their solutions are not valid physical models for all values of time. The Langevin model is valid for $t \gg \tau_{k}$, the Kolmogorov time scale, and the random displacement model is valid for $t \gg \tau$, the particle velocity decorrelation time scale. For accurate numerical solutions, $\Delta t \ll \tau$ for the Langevin model. The corresponding maximum value of $\Delta t$ for the random displacement model is a presently ill-defined function of the scale of inhomogenity of the turbulent flow field. Thomson's (1987) "simplest" solution for the Langevin model in three dimensions in nonstationary inhomogeneous Gaussian turbulence is evaluated with respect to its complexity. It is shown that the use of this solution in three dimensions is feasible if it is assumed that the turbulence is stationary and horizontally homogeneous, and that the longitudinal axis is aligned with the mean flow velocity such that there is zero mean flow laterally and vertically. This three-dimensional model is simplified to two dimensions (lateral and vertical) and to one dimension (vertical). The existing solution for one-dimensional (vertical) turbulent diffusion in non-Gaussian turbulence is presented. There are, at present, no models for diffusion in two- and three-dimensional non-Gaussian turbulence. The scaling parameters for the stable, near-neutral, and unstable atmospheric boundary later are reviewed. Parameterizations of the turbulence statistics used as model inputs are developed that give continuous values over the entire parameter space of dimensionless height $z / h$ and atmospheric stability $h / L$ proposed by Holtslag and Nieuwstadt (1986). These parameterizations include a large number of adjustable coefficients and provisional values are given for these coefficients. The Ito Calculus for integrating stochastic diffential equations is described and discussed. The finite-difference form of the Langevin Model for one-dimensional (vertical) diffusion is given as an example of the Ito rule for integration.

\section{Acknowledgements}

My work on these notes during the past five years has benefited from many discussions with my colleagues at the Lawrence Livermore National Laboratory: Ric Cederwall, Marv Dickerson (who gave me the "go ahead"), Don Ermak, Paul Gudiksen, Rolf Lange, John Nasstrom (of EG and G), and Tom Sullivan. In addition, I worked with two valued foreign colleagues: Vladimir Gavrilov of the Institute of Experimental Meteorology, Obninsk, Russia, and Brian Sawford of the CSIRO Division of Atmospheric Research, Mordialloc, Australia. 
I owe special thanks to John Nasstrom and Brian Sawford who carefully read the final draft and offered many comments and corrections. Finally, Cyndi Brandt, Raylene Cooper, Amy Henke, and Lourdes Placeres did all the word processing. Any remaining errors, typographical or otherwise, are my responsibility. 


\section{References}

Arnold, L., 1974: Stochastic differential equations: Theory and applications. John Wiley and Sons, New York.

Barnett, S., 1990: Matrices-Methods and applications. Clarendon Press, Oxford.

Boughton, B.A., J.M. Delaurentis, and W.E. Dunn, 1987: A stochastic model of particle dispersion in the atmosphere. Boundary-Layer Meteorol., 40, 147-163. 1989: 48, 443-444.

Baerentsen, J.H., and R. Berkowicz, 1984: Monte-Carlo simulation of plume diffusion in the connective boundary layer. Atmos. Environ., 18, 701-712.

Brost, R.A., and J.C. Wyngaard, 1978: A model study of the stably stratified planetary boundary layer, J. Atmos. Sci., 35, 1427-1440.

Calder, K.L., 1966; Concerning the similarity theory of Monin/Obukhov. Quart. J. R. Met. Soc. , 92, 141-146.

Caughey, S.J., and S.G. Palmer, 1979: Some aspects of turbulence structure through the depth of the convective boundary layer, Quart. J. R. Met. Soc., 105, 811-827.

Caughey, S.J., J.C. Wyngaard, and J.C. Kaimal, 1979: Turbulence in the evolving stable boundary layer, J. Atmos. Sci., 36, 1041-1052.

de Baas, A.F., and I. Troen, 1989: A stochastic equation for diffusion in inhomogeneous conditions. Physica Scripta, 40, 64-72.

Doob, J.L., 1953: Stochastic processes. John Wiley and Sons, New York.

Durbin, P.A., 1984: Comments on Paper by Wilson et al. (1981) and Legg and Raupach (1982). Boundary-Layer Meteorol., 29, 409-411.

Gardiner, C.W., 1983: Handbook of stochastic methods. Springer-Verlag, Berlin.

Gray, A.H., Jr., and T.K. Caughey, 1986: A controversy in problems involving random parametric excitation. J. Math. Phys., 44, 288-296.

Holtslag, A.A.M., and F.T.M. Nieuwstadt, 1986: Scaling the atmospheric boundary layer, BoundLayer Meteorol., 36, 201-109.

Högström, U. 1990: Analysis of turbulence structure in the surface layer with a modified similarity formulation for near neutral conditions, J. Atmos. Sci., 47, 1949-1972.

Lange, 1978: ADPIC-A three-dimensional particle-in-cell model for the dispersal of atmospheric pollutants and its comparison to regional tracer studies. J. Appl. Meteorol., 17, 320-329.

Legg, B.J., and M.R. Raupach, 1982: Markov-chain simulation of particle dispersion in inhomogeneous flows: The mean drift velocity induced by a gradient in Eulerian velocity variance. Boundary-Layer Meteorol., 24, 3-13.

Lenschow, D.H., J.C. Wyngaard, and W.T. Fennel, 1980: Mean-field and second-moment budgets in a baroclinic, convective boundary layer, J. Atmos. Sci., 37, 1313-1326. 
Lenschow, D.H., X.S. Li, C.J. Zhu, and B.B. Stankov, 1988: The stably stratified boundary layer over the Great Plains, Bound-Layer Meteorol., 42, 95-121.

Lin, C.C., and W.H. Reid, 1963: Turbulent flow, theoretical aspects. Handbuch der Physik, VIII/2, 438-523.

Luhar, A.K., and R.E. Britter, 1989: A random walk model for diffusion in inhomogeneous turbulence in a convective boundary layer. Atmos. Environ., 23, 1911-1924.

Mason, P.J., and S.H. Derbyshire, 1990: Large-eddy simulation of the stably-stratified atmospheric boundary layer, Bound-Layer Meteorol, 53, 117-162.

Moeng, C.-H., and J.C. Wyngaard, 1984: Statistics of conservative scalars in the convective boundary layer, J. Atmos. Sci., 41, 3161-3169.

Moeng, C.-H., and J.C. Wyngaard, 1989: Evaluation of turbulent transport and dissipation closures in second-order modeling, J. Atmos. Sci., 48, 2311-2330.

Monin, A.S., and A.M. Yaglom, 1971, 1975: Statistical fluid mechanics: Mechanics of turbulence, Vols. 1 and 2. MIT Press, Cambridge.

Nicholls, S., and C.J. Readings, 1979: Aircraft observations of the structure of the lower boundary layer over the sea, Quart. J. R. Met. Soc., 105, 785-802.

Nieuwstadt, F.T.M, 1984: The turbulent structure of the stable, nocturnal boundary layer, J. Atmos. Sci., 41, 2202-2216.

Obukhov, A.M., 1959: Description of turbulence in terms of Lagrangian variables. Adv. Geophys.. 6, 113-116.

Panofsky, H.A., H. Tennekes, D.H. Lenschow, and J.C. Wyngaard, 1977: The characteristics of turbulent velocity components in the surface layer under convective conditions, Bound-Layer Meteorol., 11, 355-361.

Pope, S.B., 1987: Consistency conditions for random-walk models of turbulent diffusion. Phys. Fluids, 30, 2374-2379.

Sawford, B.L., 1984: The basis for, and some limitations of the Langevin equation in atmospheric relative diffusion modelling. Atmos. Environ., 18, 2405-2411.

Sawford, B.L., 1986: Generalized random forcing in random-walk turbulent diffusion models. Phys. Fluids, 29, 3582-3585.

Sawford, B.L., and F.M. Guest, 1987: Lagrangian stochastic analysis of flux-gradient relationships in the convective boundary layer, J. Atmos. Sci., 44, 1152-1169.

Sawford, B.L., and F.M. Guest, 1988: Uniqueness and universality of Lagrangian stochastic models of turbulent diffusion. Proceedings of the 8th Symp. on Turbulence and Diffusion (Amer. Meteorol. Soc., Boston.) 96-99.

Schumann, U., 1977: Realizability of Reynolds-stress turbulence models, Phys. Fluids, 201, 721725.

Sorbjan, Z., 1990: Similarity scales and universal profiles of statistical moments in the convective boundary layer, J. Appl. Meteorol., 29, 762-775. 
Stratonovich, R.L., 1966: A new representation for stochastic integrals and equations. J. SIAM Control, 4, 362-371.

Thomson, D.J., 1984: Random walk modeling in diffusion in inhomogeneous turbulence. Quart. J.R. Met. Soc., 110, 1107-1120.

Thomson, D.J., 1987: Criteria for the selection of stochastic models of particle trajectories in turbulent flows. J. Fluid Mech., 180, 529-556.

van Dop, H.F., T.M. Nieuwstadt, and J.C.R. Hunt, 1985: Random walk models for particle displacements in inhomogeneous unsteady turbulent flows. Phys. Fluids, 28, 1639-1653.

van Kampen, N.G., 1981a: Ito versus Stratonovich. J. Stat. Phys., 24,175-187.

van Kampen, N.G., 1981b: Stochastic processes in physics and chemistry. North-Holland Publishing Company, Amsterdam.

van Ulden, A.P., and A.A.M. Holtslag, 1985: Estimation of atmospheric boundary layer parameters for diffusion applications, J. Climate Appl. Meteorol., 24, 1196-1207.

Weil, J.C., 1990: A diagnosis of the asymmetry in top-down and bottom-up diffusion using a Lagrangian stochastic model, J. Atmos. Sci., 47, 501-515.

Wilson, J.D., B.J. Legg, and D.J. Thomson, 1983: Calculation of particle trajectories in the presence of a gradient in turbulent-velocity variance. Boundary-Layer Meteorol., 27, 163-169.

Wyngaard, J.C., and O.R. Cote, 1971: The budgets of turbulent kinetic energy and temperature variance in the atmospheric surface layer, J. Atmos. Sci., 28, 190-201.

Wyngaard, J.C., O.R. Cote, and Y. Izumi 1971: Local free convection, similarity, and the budgets of shear stress and heat flux, J. Atmos. Sci., 28, 1171-1182.

Wyngaard, J.C., O.R. Cote, and K.S. Rao 1974: Modelling the atmospheric boundary layer, Advances in Geophysics, 18A, 193-211.

Langevin Notes, Part III.tex 


\section{Appendix: From Eq (4) toward Eq (32) in Thomson (1987)}

\section{Introduction}

The theme of Thomson (1987) is explicit in the title, "Criteria for the selection of stochastic models of particle trajectories in turbulent flows." He proposes the "well-mixed condition" as the principal criterion: If a tracer is initially well-mixed in a turbulent flow, it will stay that way. He then demonstrates that most other proposed criteria are equivalent to the well-mixed condition. In this Appendix, we review, and in some cases expand, his presentations of the probability density function (PDF) for turbulent flow, the Langevin and Fokker-Planck equations for stochastic models of turbulent diffusion, the well-mixed criterion, and solutions for the "drift" and "diffusion" coefficients in the stochastic differential equations. He begins with general forms of the Lagrangian and Eulerian stochastic differential equations (Eqs. (4) and (5), respectively, in his text) and ends with the simplest of the possible solutions (Eqs. (24), (32), and the line following (32)). There are few, if any, obvious hints as to how his Eq. (32) was derived. The main purpose of this Appendix is to follow the mathematical processes that lead from Eq. (4) toward Eq. (32), based on suggestions from Brian Sawford (private communications; 1992, 1993).

\section{Multivariate Gaussian Distribution for Turbulence}

The probability distribution function (PDF) for Gaussian turbulence in three dimensions given by Thomson (1987) 2.1, p531 may be written, with some changes in notation, as

$$
P_{E}=(2 \pi)^{-\frac{3}{2}}\left(\operatorname{det} \tau_{i j}\right)^{-\frac{1}{2}} \exp \left[-\frac{1}{2}\left(u_{i}-U_{i}\right)\left(\tau_{i j}\right)^{-1}\left(u_{j}-U_{j}\right)\right]
$$

in which

$$
\begin{aligned}
& \mathbf{u}=\text { the Lagrangian velocity vector, } \\
& \mathbf{U}=\left\langle\mathbf{u}_{E}\right\rangle \text { where } \\
& \mathbf{u}_{E}=\text { the Eulerian velocity vector, } \\
& \boldsymbol{\tau}_{i j}=\left\langle\left(u_{E i}-U_{i}\right)\left(u_{E j}-U_{j}\right)\right\rangle, \text { and } \\
& i, j=1,2,3
\end{aligned}
$$

Like Thomson, we use tensor notation with the Einstein summation convention. Unlike Thomson, we assume incompressible flow in the definitions of $U$ and $\boldsymbol{\tau}_{\boldsymbol{i j}}$. The meaning of $\operatorname{det} \boldsymbol{\tau}_{\boldsymbol{i j}}$ is clear: it is the determinate of the tensor $\tau_{i j}$ as a $3 \times 3$ matrix. It is not explicitly stated in words by Thomson that $\left(\tau_{i j}\right)^{-1}$ is the inverse of $\tau_{i j}$, not a simple reciprocal. The exponential term is one-half the product of the instantaneous velocity correlation and the inverse of the velocity covariance tensor. We will see later why the exponential term is written in the order $\left(u_{i}-U_{i}\right)\left(\tau_{i j}\right)^{-1}\left(u_{j}-U_{j}\right)$.

It is appropriate at this point to define the notation to be used in this Appendix:

$$
\begin{aligned}
& \tau_{i j}=\text { an element of a tensor or matrix, } \\
& \tau_{i j}=\text { a tensor, }
\end{aligned}
$$




$$
\begin{aligned}
& \left|\tau_{i j}\right|=\text { a matrix, and } \\
& \left|\tau_{i j}\right|=\text { a determinant. }
\end{aligned}
$$

Bold notation without subscripts (e.g., $\mathbf{u}, \mathbf{x}$ ) indicates a vector, which is a first-order tensor.

The literature is not always explicit about the inverse of the covariance tensor (or covariance matrix) in explaining or discussing the equivalent of Eq. (1) for multivariate PDFs. Doob (1953) II.3, pp72-73 explicitly defines a matrix as the inverse of the covariance matrix. van Kampen (1981) uses both the covariance matrix (VIII.6, pp230 and 231) and its inverse (I.6, p25) without using the term "the inverse." On the other hand, Risken (1989) 2.2.3, pp23-24 clearly defines the covariance matrix and its inverse. We will adapt the cited material in Doob in discussing the following alternative form of Eq. (1):

$$
P_{E}=(2 \pi)^{-\frac{3}{2}} \lambda^{\frac{1}{2}} \exp \left[-\frac{1}{2} \sum_{i, j=1}^{3}\left(u_{i}-U_{i}\right)\left[\lambda_{i j}\right]\left(u_{j}-U_{j}\right)\right]
$$

where

$$
\begin{aligned}
& {\left[\lambda_{i j}\right]=\left[\tau_{i j}\right]^{-1} \text { and }} \\
& \lambda=\left|\lambda_{i j}\right|=\operatorname{det}\left[\lambda_{i j}\right] .
\end{aligned}
$$

We have shifted from tensor to matrix notation, hence the explicit summation in Eq. (2) as in Doob.

Doob requires that the matrix $\left[\tau_{i j}\right]$ be "non-negative definite" and "nonsingular," and states that it is also "symmetric" (i.e., $\tau_{i j}=\tau_{j i}$ ). Only nonsingular matrices have inverses, and a (square) matrix is nonsingular if its determinant is nonzero (Searle (1982) 5.5, pp129-130). Therefore $\left[\tau_{i j}\right]$ has the inverse $\left[\lambda_{i j}\right]$ if $\left|\tau_{i j}\right| \neq 0$. The inverse $\left[\lambda_{i j}\right]$ is also nonsingular, and its determinant $\left|\lambda_{i j}\right|$ is equal to the reciprocal of $\left|\tau_{i j}\right|$ as indicated by Eqs. (1) and (2) (Searle, 5.6, pp130-131). The non-negative definite property is best explained in the following context (Searle, 3.5-3.6, pp 73-78). Let

$$
\begin{gathered}
\sum_{i, j=1}^{3}\left(u_{i}-U_{i}\right)\left[\lambda_{i j}\right]\left(u_{j}-U_{j}\right)=\sum_{i, j=1}^{3} \Delta u_{i}\left[\lambda_{i j}\right] \Delta u_{j}= \\
{\left[\Delta u_{1} \Delta u_{2} \Delta u_{3}\right]\left[\begin{array}{lll}
\lambda_{11} & \lambda_{12} & \lambda_{13} \\
\lambda_{21} & \lambda_{22} & \lambda_{23} \\
\lambda_{31} & \lambda_{32} & \lambda_{33}
\end{array}\right]\left[\begin{array}{l}
\Delta u_{1} \\
\Delta u_{2} \\
\Delta u_{3}
\end{array}\right] .}
\end{gathered}
$$

The right side of Eq. (3) is a quadratic form consisting of the product of a row vector, a symmetric matrix, and a column vector. The column vector $\Delta u_{j}$ is the transpose of the row vector $\Delta u_{i}$, so we could also use $\Delta u_{i}=\Delta \mathbf{u}$ and $\Delta u_{j}=\Delta \mathbf{u}$ as in Searle. The matrix $\left[\lambda_{i j}\right]$ is positive definite if the quadratic form is zero only if all $\Delta u=0$, and it is positive semidefinite if the quadratic form is also zero for some $\Delta u \neq 0$. These two classes together are non-negative definite. The quadratic form, with $\lambda_{i j}=\lambda_{j i}$, can be expressed as

$$
\sum_{i, j} \Delta u_{i}\left[\lambda_{i j}\right] \Delta u_{j}=\sum_{i} \Delta u_{i}^{2} \lambda_{i j}+2 \sum_{j>i} \Delta u_{i} \Delta u_{j} \lambda_{i j}
$$


With $i, j=1,2,3$, we get the nine components of a second order tensor or a $3 \times 3$ matrix. Equation (3) also illustrates the rationale for writing the exponents as shown in Eqs. (1) and (2).

The above demonstrates the central role of the inverse of the kinematic Reynolds stress tensor $\left[\boldsymbol{\tau}_{i j}\right]$ ) (or matrix $\tau_{i j}$ in the Gaussian PDF for three-dimensional turbulence. The following is the procedure for finding the inverse of a tensor (or matrix)(Searle (1982) 4.3, p93; 5.5, pp128-129; Barnett (1990) 4.1, p55; 4.3, p68)). We start with the matrix

$$
\left|\tau_{i j}\right|=\left[\begin{array}{lll}
\tau_{11} & \tau_{12} & \tau_{13} \\
\tau_{21} & \tau_{22} & \tau_{23} \\
\tau_{31} & \tau_{32} & \tau_{33}
\end{array}\right]
$$

The cofactors of $\left[\tau_{i j}\right]$ are defined as

$$
c_{i j}=(-1)^{i+j}\left[M_{i j}\right]
$$

where $\left[M_{i j}\right]$ is $\left[\tau_{i j}\right]$ with its $i$ th row and $j$ th column deleted. Then

$$
\left[c_{i j}\right]=\left[\begin{array}{lll}
c_{21} & c_{12} & c_{13} \\
c_{21} & c_{22} & c_{23} \\
c_{31} & c_{32} & c_{33}
\end{array}\right]
$$

The inverse of $\left[\tau_{i j} \mid\right.$ is the product of the reciprocal of $\left|\tau_{i j}\right|$ and the transpose of the matrix $\left[c_{i j}\right]$,

$$
\left.\left[\lambda_{i j}\right]=\left[\tau_{i j}\right]^{-1}=\mid \tau_{i j}\right]^{-1}\left[\left.c_{j i}|=| \tau_{i j}\right|^{-1}\left[\begin{array}{lll}
c_{11} & c_{21} & c_{31} \\
c_{12} & c_{22} & c_{32} \\
c_{13} & c_{23} & c_{33}
\end{array}\right]\right.
$$

It follows that

$$
\lambda_{i j}=c_{j i}\left|\tau_{i j}\right|^{-1}
$$

\section{Brief Summary of Thomson's Development of the "Simplest" Solution}

It's assumed in Thomson (1987) 2.3, pp533-534 that the evolution of $(\mathbf{x}, \mathbf{u})$ is a Markov process and that $\mathbf{x}$ and $\mathbf{y}$ are continuous functions of $\iota$ with $d \mathbf{x} / d t=\mathbf{u}$ (see also Doob (1953) VI.3, p273-275). Thomson's Eqs. (4a-b) are

$$
\begin{gathered}
d u_{i}=a_{i}(\mathbf{x}, \mathbf{u}, t) d t+b_{i j}(\mathbf{x}, \mathbf{u}, t) d W_{j}(t) \\
d \mathbf{x}=\mathbf{u} d t
\end{gathered}
$$

Here

$\mathbf{x}=$ displacement

$\mathbf{u}=$ Lagrangian velocity, and 
$a W_{j}(t)=$ an incremental Wiener process.

This process is Gaussian with

$$
\begin{gathered}
\left\langle d W_{j}(t)\right\rangle=0 \text { and } \\
\left\langle d W_{i}(t) d W_{j}(s)\right\rangle=\delta_{i j} \delta(t-s) d t d s
\end{gathered}
$$

where $\delta_{i j}$ is the Kronecker delta and $\delta(t-s)$ is the Dirac delta function with dimension $t^{-1}$. The dimension of both sides of Eq. (11b) is $t$.

We will make extensive use of Thomson's Eq. (5), the forward Kolmogorov or Fokker-Planck equation,

$$
\frac{\partial P_{E}}{\partial t}+\frac{\partial}{\partial x_{i}}\left(u_{i} P_{E}\right)=-\frac{\partial}{\partial u_{i}}\left(a_{i} P_{E}\right)+\frac{\partial^{2}}{\partial u_{i} \partial u_{j}}\left(\frac{1}{2} b_{i j} b_{j k} P_{E}\right)
$$

where the symbols have the same meaning as in Eqs. (1), (2), and (10a-b).

The well-mixed condition (3.1, pp534-535) leads to Thomson's Eqs. (9a-b) and (10) which satisfy Eqs. (10a-b) and (12) above:

$$
\begin{gathered}
a_{i} P_{E}=\frac{\partial}{\partial u_{i}}\left(\frac{1}{2} b_{i j} b_{j k} P_{E}\right)+\phi_{i}(\mathbf{x}, \mathbf{u}, t), \\
\frac{\partial \phi_{i}}{\partial u_{i}}=-\frac{\partial P_{E}}{\partial t}-\frac{\partial}{\partial x_{i}}\left(u_{i} P_{E}\right), \text { and } \\
\phi \rightarrow 0 \text { as }|\mathbf{u}| \rightarrow \infty
\end{gathered}
$$

Thomson introduces the Lagrangian structure function $(4.1, \mathrm{p} 543)$ and uses it in combination with Eq. (10a) to get the result given in Thomson's Eq. (24),

$$
\left\langle b_{i j} b_{j k}\right\rangle=\delta_{i j} C_{o} \epsilon
$$

for small time intervals greater than the Kolmogorov time scale but within the Kolmogorov inertial range. $C_{o}$ is the universal constant associated with the Lagravgian structure function, and $\epsilon$ is the mean rate of turbulence kinetic energy dissipation with $\epsilon(\mathbf{x}, t)$.

Finally, Thomson writes "In more than one dimension there are many possible choices for 0 of which the simplest is [5.1, p 547, Eq. (32)]." We rewrite this equation as

$$
\begin{aligned}
\frac{\phi_{i}}{P_{E}} & =\frac{\partial U_{i}}{\partial t} \\
& +\frac{1}{2} \frac{\partial \tau_{\mathfrak{U}}}{\partial x_{j}}+U_{j} \frac{\partial U_{i}}{\partial x_{j}}+\left(u_{j}-U_{j}\right) \frac{\partial U_{i}}{\partial x_{j}} \\
& +\frac{1}{2} \lambda_{\mathbf{j k}} \frac{\partial \tau_{\mathbf{k} \mathbf{k}}}{\partial t}\left(u_{j}-U_{j}\right) \\
& +\frac{1}{2} \lambda_{\mathbf{j k}}\left(U_{m} \frac{\lambda \tau_{\mathbf{l k}}}{\partial x_{m}}\right)\left(u_{j}-U_{j}\right) \\
& +\frac{1}{2} \lambda_{\mathbf{j k}}\left[\left(u_{m}-U_{m}\right) \frac{\partial \tau_{\mathbf{k} \mathbf{k}}}{\partial x_{m}}\right]\left(u_{j}-U_{j}\right)
\end{aligned}
$$


The first term on the right has one index, the three terms on the second line have two indices, the term in the third line has three, and the terms in the last two lines have four. Again, the Einstein summation convention is used. The solution for $a_{i}$ is given in the first line below Eq. (32) on p547 which we write as

$$
a_{i}=-\frac{1}{2} \delta_{i j} C_{o} \varepsilon \lambda_{j k}\left(u_{k}-U_{k}\right)+\frac{\phi_{i}}{P_{E}}
$$

in which the quantity $\delta_{i j} C_{o} \varepsilon$ comes from Eq (15). There are no obvious hints in Thomsons paper to indicate how the above "simplest" solution was obtained. The following is based on suggestions from Brian Sawford (private communications; 1992, 1993).

\section{Solutions for Turbulent Diffusion in Three Dimensions}

\section{Partial Derivatives of the Multivariate Gaussian Distribution}

For convenience, we rewrite Eq. (2) as

$$
\ln P_{E}=-\frac{3}{2} \ln (2 \pi)+\frac{1}{2} \ln \lambda-\frac{1}{2}\left(u_{i}-U_{i}\right) \lambda_{i j}\left(u_{j}-U_{j}\right) .
$$

where

$$
\begin{aligned}
\mathbf{u} & =\text { Langrangian velocity vector, } \\
\mathbf{u}_{\mathbf{E}} & =\text { Eulerian velocity vector, } \\
\mathbf{U} & =\mathbf{U}(\mathbf{x}, t)=\left\langle\mathbf{u}_{\mathbf{E}}\right\rangle, \\
\lambda & =\left|\lambda_{i j}\right|, \\
\lambda_{i j} & =\left(\tau_{i j}\right)^{-1}, \\
\tau_{i j} & =\tau_{i j}(\mathbf{x}, t)=\left\langle\left(u_{E i}-U_{i}\right)\left(u_{E j}-U_{j}\right),\right.
\end{aligned}
$$

and $\mathbf{x}$ and $\mathbf{u}$ are independent variables.

For corivenience in Eq. (18) and the following, we do not denote a tensor like $\tau_{i j}$ or $\lambda_{i j}$ with bold fare type. Footnote 4 on p121 of Arfken (1985) is appropriate in this context: "It is common practice to refer to a tensor $\mathbf{A}$ by specifying a typical component $A_{i j}$. As long as the reader refrains from writing nonsense such as $\mathbf{A}=A_{i j}$, no harm is done."

We differentiate Eq. (18) with respect to $\mathbf{x}$, $\mathbf{u}$, and t. First,

$$
\frac{\partial}{\partial u_{i}}\left(\ln P_{E}\right)=-\lambda_{i k}\left(u_{k}-U_{k}\right)
$$

as shown in Searle (1982) 12.8.c, pp329-330 for the differential of a quadratic form. Second, 


$$
\begin{aligned}
\frac{\partial}{\partial t}\left(\ln P_{E}\right) & =\frac{1}{2} \frac{\partial}{\partial t}(\ln \lambda)+\left(u_{j}-U_{j}\right) \lambda_{j k} \frac{\partial U_{k}}{\partial t} \\
& -\frac{1}{2}\left(u_{j}-U_{j}\right)\left(u_{k}-U_{k}\right) \frac{\partial \lambda_{j k}}{\partial t} .
\end{aligned}
$$

Third,

$$
\begin{aligned}
\frac{\partial}{x_{i}}\left(\ln P_{E}\right) & =\frac{1}{2} \frac{\partial}{\partial x_{i}}(\ln \lambda)+\left(u_{j}-U_{j}\right) \lambda_{j k} \frac{\partial U_{k}}{\partial x_{i}} \\
& -\frac{1}{2}\left(u_{j}-U_{j}\right)\left(u_{k}-U_{k}\right) \frac{\partial \lambda_{j k}}{\partial x_{i}}
\end{aligned}
$$

\section{The Solutions for the "Fading Memory" Term}

It is relatively simple to obtain the "fading memory" term for the Langevin model. From Eqs. (13a), (15) and (19) we get

$$
a_{i}=-\left(\frac{C_{o} \varepsilon}{2}\right) \lambda_{i k}\left(u_{k}-U_{k}\right)+\frac{\phi_{i}}{P_{E}}
$$

which is equivalent to part of Thomson's solution, Eq. (17) above. The "fading memory" term is the first term on the right. The remainder of our quest to obtain the solution for the "drift" term $\phi_{i} / P_{E}$ is much more difficult.

\section{Solutions for Components of the "Drift" Term}

As indicated by the above heading, there is more than one possible solution for the "drift" term in the Langevin model for more than one dimenson. We consider the three-dimensional case in the following. We begin by rewriting Eq. (13b) and use the incompressibility condition $\partial u_{i} / \partial x_{i}=0$ to get

$$
\frac{1}{P_{E}} \frac{\partial \phi_{i}}{\partial u_{i}}=-\frac{\partial}{\partial t}\left(\ln P_{E}\right)-u_{i} \frac{\partial}{\partial x_{i}}\left(\ln P_{E}\right)
$$

We expand $u_{i}$ in the second term on the right to $\left(u_{i}-U_{i}+U_{i}\right)$ and combine the result with Eqs. (20) and (21) to get 


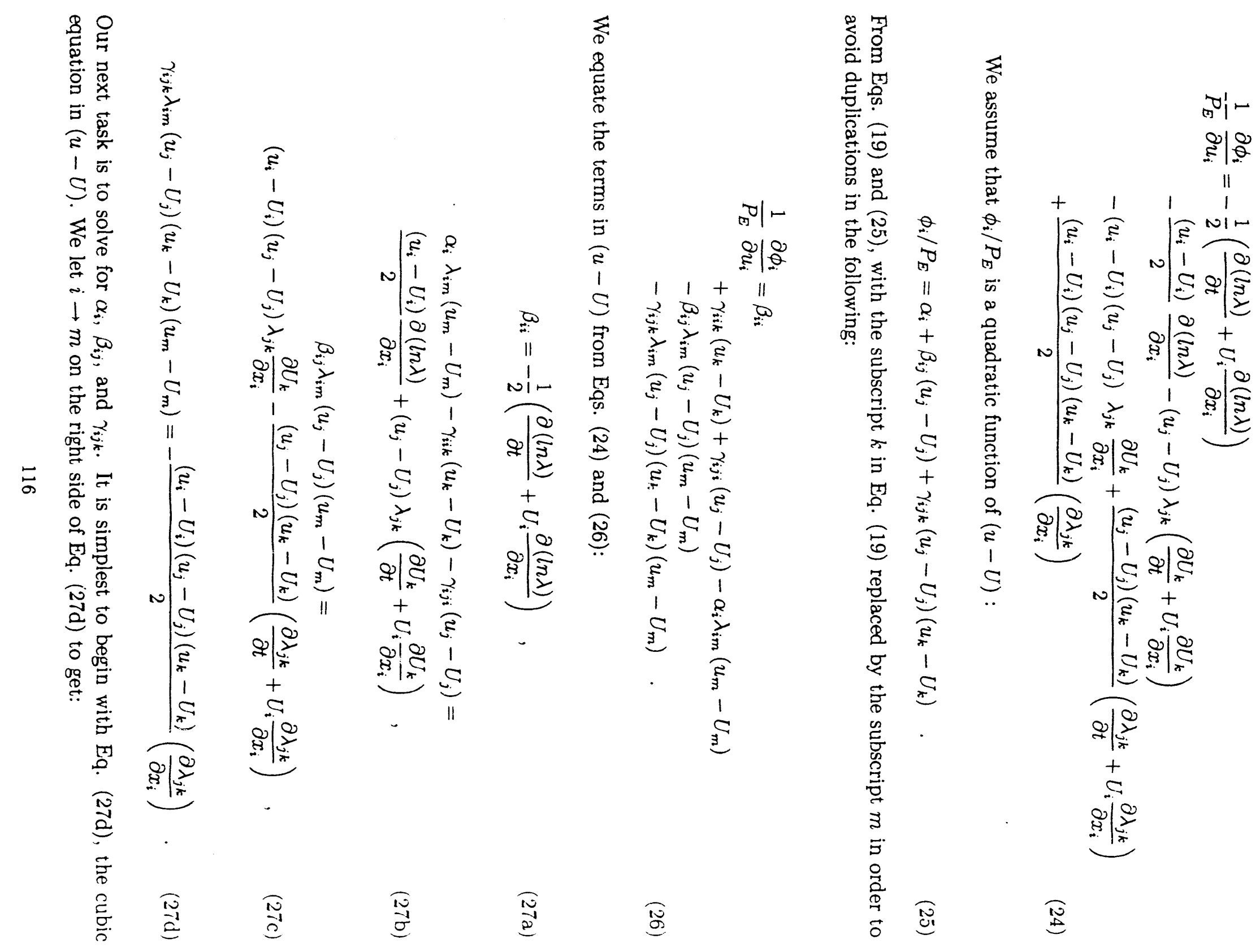




$$
\gamma_{i j k} \lambda_{i m}\left(u_{j}-U_{j}\right)\left(u_{k}-U_{k}\right)\left(u_{m}-U_{m}\right)=-\frac{\left(u_{j}-U_{j}\right)\left(u_{k}-U_{k}\right)\left(u_{m}-U_{m}\right)}{2}\left(\frac{\partial \lambda_{j k}}{\partial x_{m}}\right)
$$

The coefficients of the cubic terms on both sides of Eq. (28) are equal for all combinations of $j, k, m=1,2,3$ so

$$
\gamma_{i j k}=-\frac{\lambda_{i m}^{-1}}{2} \frac{\partial \lambda_{j k}}{\partial x_{m}}
$$

This is the first solution for $\gamma_{i j k}$. The second comes from the cyclic permutation of the indices $j \rightarrow k \rightarrow m \rightarrow j$ on the right side of Eq. (28):

$$
\gamma_{i j k}=-\frac{\lambda_{i m}^{-1}}{2} \frac{\partial \lambda_{k m}}{\partial x_{j}}
$$

The third, and last, comes from a further cyclic permutation of the same indices on the same side of Eq. (28):

$$
\gamma_{i j k}=-\frac{\lambda_{i m}^{-1}}{2} \frac{\partial \lambda_{m j}}{\partial x_{k}}
$$

The above results are part of the reason that, in more than one dimension, there is no unique solution for $\phi_{i} / P_{E}$ as stated on pp544 and 547 of Thomson (1987).

We continue in a similar manner with the quadratic equation in $(u-U)$, Eq. (27c). We change $i \rightarrow m$ in the fiist term on the right and $k \rightarrow m$ in the second, in order to get both sides in terms of $\left(u_{j}-U_{j}\right)\left(u_{m}-U_{m}\right)$. In addition, to avoid future difficulty, we change $i \rightarrow k$ in the second term on the right. The result is

$$
\beta_{i j} \lambda_{i m}\left(u_{j}-U_{j}\right)\left(u_{m}-U_{m}\right)=\left(u_{j}-U_{j}\right)\left(u_{m}-U_{m}\right)\left[\lambda_{j k} \frac{\partial U_{k}}{\partial x_{m}}-\frac{1}{2}\left(\frac{\partial \lambda_{j m}}{\partial t}+U_{k} \frac{\partial \lambda_{j m}}{\partial x_{k}}\right)\right]
$$

The coefficients of the quatratic terms on both sides of Eq. (30) must be equal for all combinations of $j, m=1,2,3$. The first solution for $\beta_{i j}$ is obtained by multiplying both sides of Eq. (30) by $\lambda_{i m}^{-1}=\tau_{i m}$ :

$$
\beta_{i j}=\lambda_{i m}^{-1} \frac{\partial U_{k}}{\partial x_{m}} \lambda_{j k}-\frac{\tau_{i m}}{2}\left(\frac{\partial \lambda_{j m}}{\partial t}+U_{k} \frac{\partial i^{\prime}, m}{\partial x_{k}}\right) .
$$

The second solution is obtained by means of the cyclic permustation $j \rightarrow m \rightarrow j$ on the right side of Eq. (30):

$$
\beta_{i j}=\frac{\partial U_{i}}{\partial x_{j}}-\frac{\tau_{i m}}{2}\left(\frac{\partial \lambda_{j m}}{\partial t}+U_{k} \frac{\partial \lambda_{j m}}{\partial x_{k}}\right)
$$


The second term on the right in Eq. (31b) is unchanged from that in Eq. (31a) because $\lambda_{j m}=\lambda_{m j}$. The first term is a consequence of the following relationships (Jaeger, 1966, 3.6, pp45-46):

$$
\lambda_{i m}^{-1} \lambda_{m k} \frac{\partial U_{k}}{\partial x_{j}}-\delta_{i k} \frac{\partial U_{k}}{\partial x_{j}}=\frac{\partial U_{i}}{\partial x_{j}}
$$

where $\delta_{i k}$ is the Kronecker delta as a second-order tensor.

Now we consider Eq. (27b) which is linear in $(u-U)$. On the left, we let $m \rightarrow j$ in the first term and $k \rightarrow j$ in the second. On the right, we let $i \rightarrow j$ in the first term and, to avoid future difficulty, $i \rightarrow m$ in the second. The result is

$$
\left(\alpha_{i} \lambda_{i j}-\gamma_{i i j}-\gamma_{i j i}\right)\left(u_{j}-U_{j}\right)=\left[\frac{1}{2} \frac{\partial(\ln \lambda)}{\partial x_{j}}+\lambda_{j k}\left(\frac{\partial U_{k}}{\partial_{t}}+U_{m} \frac{\partial U_{k}}{\partial x_{m}}\right)\right]\left(u_{j}-U_{j}\right) .
$$

The coefficient of $\left(u_{j}-U_{j}\right)$ on the left must be equal to that on the right for all values of $j=1,2,3$, so we equate these coefficients,

$$
\alpha_{i} \lambda_{i j}-\gamma_{k k j}-\gamma_{k j k}=\frac{1}{2} \frac{\partial(l n \lambda)}{\partial x_{j}}+\lambda_{j k}\left(\frac{\partial U_{k}}{\partial_{t}}+U_{m} \frac{\partial U_{k}}{\partial x_{m}}\right)
$$

Note that we let $i \rightarrow k$ in the second and third terms on the left to avoid future difficulty. We multiply both sides of Eq. (34) by $\lambda_{i j}^{-1}=\tau_{i j}$ and move the terms with $\gamma_{k k j}$ and $\gamma_{k j k}$ to the right.

$$
\alpha_{i}=\lambda_{i j}^{-1}\left(\gamma_{k k j}+\gamma_{k j k}\right)+\frac{\tau_{i j}}{2} \frac{\partial(\ln \lambda)}{\partial x_{j}}+\lambda_{i j}^{-1} \lambda_{j k}\left(\frac{\partial U_{k}}{\partial t}+U_{m} \frac{\partial U_{k}}{\partial x_{m}}\right)
$$

We apply the principles of Eq. (32) to the last term on the right, after which we let $m \rightarrow j$ in this term:

$$
\alpha_{i}=\left(\gamma_{k k j}+\gamma_{k j k}\right) \lambda_{i j}^{-1}+\frac{\tau_{i j}}{2} \frac{\partial \ln (\lambda)}{\partial x_{j}}+\left(\frac{\partial U_{i}}{\partial t}+U_{j} \frac{\partial U_{i}}{\partial x_{j}}\right)
$$

We have changed the sequenee of the components of the first term on the right in going from Eq. (35) to Eq. (36) in preparation for the following. We note that there are more than one solution for $\alpha_{\mathrm{t}}$ because of the multiple solutions for $\gamma_{i j k}$ given by Eq. $(29 a-c)$.

From Eq. (29a), we get the first solution for the first term in Eq. (36),

$$
\left(\gamma_{k k j}+\gamma_{k j k}\right) \lambda_{i j}^{-1}=-\lambda_{k m}^{-1} \frac{\partial \lambda_{j k}}{\partial x_{m}} \lambda_{i j}^{-1}=\frac{\partial \lambda_{i m}^{-1}}{\partial x_{m}}
$$

The second solution comes from Eq. (29b),

$$
\left(\gamma_{k k j}+\gamma_{k j k}\right) \lambda_{i j}^{-1}=\frac{1}{2}\left(-\lambda_{k m}^{-1} \frac{\partial \lambda_{j m}}{\partial x_{k}} \lambda_{i j}^{-1}-\lambda_{k m}^{-1} \frac{\partial \lambda_{k m}}{\partial x_{j}} \lambda_{i j}^{-1}\right)=\frac{1}{2}\left(\frac{\partial \lambda_{i k}^{-1}}{\partial x_{k}}-\lambda_{i j}^{-1} \frac{\partial(l n \lambda)}{\partial x_{j}}\right)
$$


We also get the second solution from Eq. (29c),

$$
\left(\gamma_{k k j}+\gamma_{k j k}\right) \lambda_{i j}^{-1}=\frac{1}{2}\left(-\lambda_{k m}^{-1} \frac{\partial \lambda_{m k}}{\partial x_{j}} \lambda_{i j}^{-1}-\lambda_{k m}^{-1} \frac{\partial \lambda_{m j}}{\partial x_{k}} \lambda_{i j}^{-1}\right)=\frac{1}{2}\left(\lambda_{i j}^{-1} \frac{\partial(\ln \lambda)}{\partial x_{j}}+\frac{\partial \lambda_{i k}^{-1}}{\partial x_{k}}\right)
$$

Let us explain the results in Eqs. $(37 \mathrm{a}-\mathrm{c})$. First, for the precedure in Eq. (37a) and similar parts of Eqs. $(37 \mathrm{~b}-\mathrm{c})$;

$$
\frac{\partial\left(\lambda_{j k} \lambda_{i j}^{-1}\right)}{\partial x_{m}}=\frac{\partial \delta_{i k}}{\partial x_{m}}=0=\lambda_{j k} \frac{\partial \lambda_{i j}^{-1}}{\partial x_{m}}+\lambda_{i j}^{-1} \frac{\partial \lambda_{j k}}{\partial x_{m}}
$$

From the right side of Eq. (38a) multiplied by $\lambda_{k m}^{-1}$,

$$
-\lambda_{k m}^{-1} \frac{\partial \lambda_{j k}}{\partial x_{m}} \lambda_{i j}^{-1} \lambda_{j k} \frac{\partial \lambda_{i j}^{-1}}{\partial x_{m}}=\delta_{j m} \frac{\partial \lambda_{i j}^{-1}}{\partial x_{m}}=\frac{\partial \lambda_{i m}^{-1}}{\partial x_{m}}
$$

The above in Eq. (38a-b) is equivalent to Searle (1982) 12.10.c, p 335. The material in 12.10.d, pp337-338 of Searle is applicable to the other parts of the solution common to Eqs. $(37 \mathrm{~b}-\mathrm{c})$. In our tensor notation (see also 2.8. $\mathrm{k}$, pp45-46 in Searle), the differential of a determinate may be written as

$$
\frac{\partial(\ln \lambda)}{\partial x_{j}}=\left(\lambda_{k n}^{-1} \frac{\partial \lambda_{k m}}{\partial x_{j}}\right)=\lambda_{k m}^{-1} \frac{\partial \lambda_{k m}}{\partial x_{j}}
$$

With $k, m=1,2,3$, there are nine terms in the expansion of the right side of Eq. (39). We now have all the components for solution for $\phi_{i} / P_{E}$ : Eqs. (25), $(29 \mathrm{a}-\mathrm{c}),(31 \mathrm{a}-\mathrm{b}),(36)$, and $(37 \mathrm{a}-\mathrm{c})$.

For the sake of completeness, let us consider the relation between Eqs. (31a-b) for $\beta_{i j}$ and Eq. (27a) for $\beta_{i i}$, If we let $\beta_{i j} \rightarrow \beta_{i i}$, Eqs. $(31 \mathrm{a}-\mathrm{b})$ reduce to the following if we apply the incompressible flow condition $\partial U_{i} / \partial x_{i}=0$ :

$$
\beta_{i i}=-\frac{\lambda_{i m}^{-1}}{2}\left(\frac{\partial \lambda_{i m}}{\partial t}+U_{k} \frac{\partial \lambda_{i m}}{\partial x_{k}}\right)
$$

This is equivalent to Eq. (27a) and is consistent with Eq. (39). 


\section{The Borgas and Thomson Solutions}

In the preceding on solutions for $\phi_{i} / P_{E}$ as difined by Eq. (25), we get three solutions for $\gamma_{i j k}$, two solutions for $\beta_{i j}$, and two solutions for $\alpha_{i}$. All are mathematically valid. An unanswered question at the time of writing is, "Which solution is closest to physical reality?"

Let us begin with the first solution for $\gamma_{i j k}$, Eq. (29a); the first solution for $\beta_{i} j$, Eq. (31a); and the first solution for $\alpha_{i}$ a combination of Eqs. (36) and (37a), with Eq. (37a) being based on Eq. (29a). The result is, after making some use of the relation $\tau_{i j}=l a m b d a_{i j}^{-1}$ and changing a few indices,

$$
\begin{aligned}
\frac{\phi_{i}}{P_{E}} & =\frac{\partial U_{i}}{\partial t}+U_{j} \frac{\partial U_{i}}{\partial x_{j}}+\frac{\partial \tau_{i j}}{\partial x_{j}}+\frac{\tau_{i m}}{2} \frac{\partial(\ln \lambda)}{\partial x_{m}} \\
& +\left[\lambda_{i m}^{-1} \frac{\partial U_{k}}{\partial x_{m}} \lambda_{j k}-\frac{\tau_{i m}}{2}\left(\frac{\partial \lambda_{j m}}{\partial t}+U_{k} \frac{\partial \lambda_{j m}}{\partial x_{k}}\right)\right]\left(u_{j}-U_{j}\right) \\
& -\left[\frac{\tau_{i m}}{2} \frac{\partial \lambda_{j k}}{\partial x_{m}}\right]\left(u_{j}-U_{j}\right)\left(u_{k}-U_{k}\right) .
\end{aligned}
$$

this result is equivalent to the solution obtained by Michael Borgas (Eq. (10) in Sawford and Guest (1987)).

We continue with the second solution for $\gamma_{i j k}$, Eq. (29b); the second solution for $\beta_{i j}$, Eq. (31b); and the second solution for $\alpha_{i}$, a combination of Eqs. (36) and (37b), with Eq. (37b) being based on Eq. (29b). The result is

$$
\begin{aligned}
\frac{\phi_{i}}{P_{E}} & =\frac{\partial U_{i}}{\partial t}+U_{j} \frac{\partial U_{i}}{\partial x_{j}}+\frac{1}{2} \frac{\partial \tau_{i j}}{\partial x_{j}} \\
& +\left[\frac{\partial U_{i}}{\partial x_{j}}-\frac{\tau_{i m}}{2}\left(\frac{\partial \lambda_{j m}}{\partial t}+U_{k} \frac{\partial \lambda_{j m}}{\partial x_{k}}\right)\right]\left(u_{j}-U_{j}\right) \\
& -\left[\frac{\tau_{i m}}{2} \frac{\partial \lambda_{k m}}{\partial x_{j}}\right]\left(u_{j}-U_{j}\right)\left(u_{k}-U_{k}\right) .
\end{aligned}
$$

The third solution for $\gamma_{i j k}$, Eq. (29c); the second solution for $\beta_{i j}$, Eq. (31b); and the third solution for $\alpha_{i}$, a combination of Eqs. (36) and (37c), with Eq. (37c) being based on Eq. (29c) is equivalent to the above. The above result, Eq. (42), is equivalent to Eq. (16), Thomson's "simplest" solution (Eq. (32) in Thomson (1987) and Eq. (9) in Sawford and Guest (1987)).

\section{Reduction to One-Dimensional Turbulence}

For the one-dimensional case, $\mathrm{Eq}(1)$ reduces to

$$
P_{E}=(2 \pi)^{-\frac{1}{2}} \sigma^{-1} \exp \left[-\frac{1}{2}(u-U)^{2} \sigma^{-2}\right]
$$


where

$$
\begin{aligned}
u & =\text { the Lagrengian velocity } \\
U & =\left\langle u_{E}\right\rangle \text { where } \\
u_{E} & =\text { the Eulerian velocity, and } \\
\sigma^{2} & =\left\langle\left(u_{E}-U\right)^{2}\right\rangle .
\end{aligned}
$$

We do not use tensor notation because we have only zeroth- and first-order tensors (scalars and vectors). The material associated with Eqs (2)-(9) for the inverse of a tensor or matrix reduces to the simple reciprocal relation

$$
\lambda=\tau^{-1}=\sigma^{-2}
$$

Equations (10)-(15) are also simplified by setting all subscripts equal to $i$ and then dropping them because they are superfluous. The results are the more familiar forms of these equations.

The one-dimensional version of Eq. (17) is, using the identities of Eq (44),

$$
a=-\frac{C_{o} \varepsilon}{2 \sigma^{2}}(u-U)+\frac{\phi}{P_{E}}
$$

Similarly, the one-dimensional version of Eq. (41) is

$$
\begin{aligned}
\frac{\phi}{P_{E}} & =\frac{\partial U}{\partial t}+U\left[\frac{\partial U}{\partial x}=0\right]+\frac{\partial \sigma^{2}}{\partial x}+\frac{\sigma^{2}}{2}\left(\frac{1}{\left(\sigma^{2}\right)^{-1}} \frac{\partial\left(\sigma^{2}\right)^{-1}}{\partial x}\right) \\
& +\left\{\sigma^{2}\left[\frac{\partial U}{\partial x}=0\right] \sigma^{-2}-\frac{\sigma^{2}}{2}\left(\frac{\partial\left(\sigma^{2}\right)^{-1}}{\partial t}+U \frac{\partial\left(\sigma^{2}\right)^{-1}}{\partial x}\right)\right\}(u-U) \\
& -\frac{\sigma^{2}}{2} \frac{\partial\left(\sigma^{2}\right)^{-1}}{\partial x}(u-U)^{2}
\end{aligned}
$$

the terms enclosed by [ ] in the above are equal to zero because we assume incompressibility. The next step in the simplification involves the $\left(\sigma^{2}\right)^{-1}$ quantities:

$$
\begin{aligned}
\frac{\phi}{P_{E}}=\frac{\partial U}{\partial t}+\frac{1}{2} \frac{\partial \sigma^{2}}{\partial x} & +\frac{1}{2 \sigma^{2}}\left(\frac{\partial \sigma^{2}}{\partial t}+U \frac{\partial \sigma^{2}}{\partial x}\right)(u-U) \\
& -\frac{1}{2 \sigma^{2}} \frac{\partial \sigma^{2}}{\partial x}(u-U)^{2}
\end{aligned}
$$

which is identical to Eq (30) in Thomson (1987), his solution for the well-mixed condition in one dimension. Thomson's "simplest solution" in Eq. (16) and (42) also reduces in one dimension to Eq (46b). 


\section{References}

Arfken, G., 1985: Mathematical methods for physicists. 3rd ed. Academic Press, Orlando, Florida.

Barnett, S., 1990: Matrices-Methods and applications. Clarendon Press, Oxford.

Jaeger, L.G., 1966: Cartesian tensors in engineering science. Pergamon Press, Oxford.

Sawford, B.L., and F.M. Guest, 1988: Uniqueness and universality of Lagrangian stochastic models of turbulent diffusion. Proceedings of the 8th Symp. on Turbulence and Diffusion. Amer. Meteorol. Soc., Boston, 96-99.

Searle, S.R., 1982: Matrix algebra useful for statistics.,John Wiley and Sons, New York.

Thomson, D.J., 1987: Criteria for the selection of stochastic models of particle trajectories in turbulent flows. J. Fluid Mech., 180, 529-556.

Langevin Notes, Appen.tex 


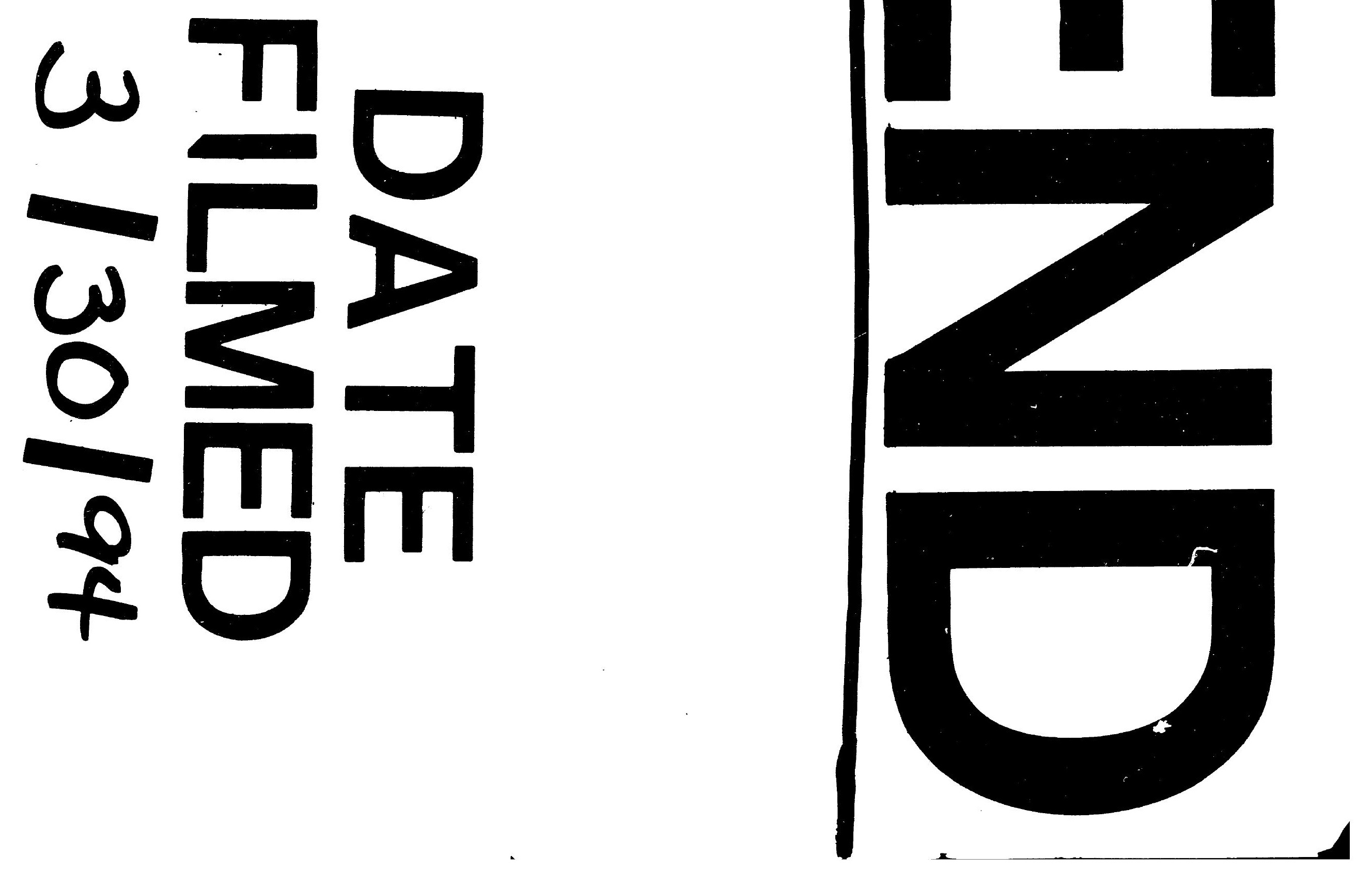




\author{
L

